\title{
ÜBER WINKELTREUE UND STRECKENTREUE ABBILDUNG AN EINEM PUNKTE UND IN DER EBENE.
}

\author{
Von Robert Remak (Berlin).
}

Adunanza del 27 lugho 1913

EIN L E I T U N G.

Es sei die komplexe von o verschiedene Zahl

$$
a=r(\cos x+i \sin x)
$$

in der Gauss'schen Zahlenebene durch einen Punkt dargestellt. Die Entfernung dieses Punktes vom Nullpunkte ist

$$
|a|=r .
$$

Die Strecke o ... $a$ bildet mit der Axe der positiven reellen Zahlen den Winkel $\boldsymbol{\alpha}$. Ich will setzen

$$
\operatorname{sgn} a=\cos \alpha+i \sin \alpha
$$

(Lies Signum $a$ ). Für reelle $a$ ist $\operatorname{sgn} a$ gleich den Vorzeichen von $a$. Diese Grösse ist also nur von der Richtung der Strecke o $\ldots a$ abhängig. Es ist für alle $a \neq 0$

ferner

$$
a=\operatorname{sgn} a \cdot|a| \text {, }
$$

$$
|\operatorname{sgn} a|=\mathrm{I},
$$

$$
\operatorname{sgn} a \cdot \operatorname{sgn} b=\operatorname{sgn}(a b) \text {. }
$$

Diese Gleichung gilt allgemein, wenn man formal setzt

Durch eine Funktion

$$
\operatorname{sgn} \mathrm{o}=\mathrm{o} .
$$

$$
u=f(\tilde{)})
$$

wird die $z$-Ebene auf die $u$-Ebene abgebildet. Ich spreche hier und im Folgenden stets von Funktionen im allgemeinsten Sinne: jedem Werte $z$ ist ein Wert $u$ zugeordnet. Den Funktionen kommen keine weiteren Eigensihaften (der Stetigkeir, Differenzierbarkeit usw.) zu, als die jedesmal ausdrücklich erwähnten. Es wird sich gerade darum handeln, eine Reihe von Bedingungen daraufhin zu untersuchen, ob man aus ihnen schliessen kann, dass die ihnen genügende Funktion eine analytische ist. 
Es bieten sich nun zwei Möglichlieiten einer Detinition der Streckentreue an einem Punkte $z=z_{1}$. Entweder man verlangt nur, dass die Länge aller von $\tau_{w}$ ansgebenden hinreichend kleinen Streiken mit heliehiger Genauigkeit in einem festen Verhältnis zur Länge der entsprechenden Strecken der $\|$-Ebene stehen, oder mus rerlangt dasselbe . für all' Strecken, deren beide Endpunkte in hinreichender Nathe ron $i_{0}$ liegen. Die erste Forderung lauter:

$$
\lim _{i_{1}=i_{0}} \frac{\left|f\left(\tilde{z}_{1}\right)-f\left(\tilde{i}_{0}\right)\right|}{\left|i_{1}-\tilde{i}_{0}\right|}=m
$$

wo $m$ eine reelle positive Zahl. Die zweite Forderung lautet:

$$
\lim _{\substack{i=1 \\ i=0 \\ i=0}} \frac{\left|f\left(i_{2}\right)-f\left(\tilde{i}_{1}\right)\right|}{\left|\tilde{i}_{2}-\tilde{i}_{1}\right|}=m .
$$

Hierin, wie in allen tolgenden Doppellimites sei zulïssig, dass einer der beiden Werte $z_{1}$ oder $i_{2}$ gleich $i_{0}$.

Analog kann man für die Winlieltreue verlangen, dass nur alle ron io ausgrebenden hinteichend kleinen Srecken mit beliebiger Genauigkeit einen festen Winkel gegen die entsprechenden Strectien der "l-Ebene bilden, oder man verlangt dasselbe für alle Strecken, deren beide Endpunkte in hinreichender Nïhe ron zu liegen. Die erste Forderung lautet:

$$
\lim _{i_{1}=i_{0}} \frac{\operatorname{sgn}\left(f\left(i_{1}\right)-f\left(z_{0}\right)\right)}{\operatorname{sgn}\left(i_{1}-z_{0}\right)}=s,
$$

wo $|s|=\mathrm{I}$. Die zweite Forderung lautet:

$$
\lim _{\substack{i=i o \\ i=i \\ i=i 0}} \frac{\operatorname{sgn}\left(f\left(\tilde{i}_{2}\right)-f\left(\tilde{i}_{1}\right)\right)}{\operatorname{sgn}\left(\tilde{i}_{2}-\tilde{i}_{1}\right)}=s .
$$

Wenn (I) und (3) gleichzeirig existieren, so ist

$$
\lim _{i_{1}=i_{0}} \frac{f\left(z_{1}\right)-f\left(\tilde{i}_{0}\right)}{z_{1}-i_{0}}=m . s,
$$

es existiert also der gewöhnliche Differentialquotient. Wenn (2) und (4) gleichzeitig existieren, so ist

$$
\lim _{\substack{i=i=0 \\ i=i 0}} \frac{f\left(\tilde{i}_{2}\right)-f\left(\tilde{i}_{1}\right)}{z_{2}-\tilde{i}_{1}}=\text { m.s. }
$$

Lch will in diesem Falle sagen, $f(i)$ besitze an der Stelle $z=i$, einen erweiterten Differentialquotienten. Entspreshend bezeichne ich die Limites (I) mit "gewöhnlicher absoluter Differentialquotient", (2) mit "erweiterter absoluter Differentialquotient ", (3) mit "gewöhnlicher Signun-Differentialquotient", (4) mit "erweiterter SignumDifferentialquotient ". Durch Kombination aller dieser Voraussetzungen ergibe sich fol- 
gendes Schema, in dem jedesmal diejenigen der Limites ( 1 ) bis (6) bezeichnet sind, deren Existenz vorausgesetzt wird.

\begin{tabular}{|c|c|c|c|c|} 
& & \multicolumn{2}{|c|}{ Der Signum-Differentialquotient existiere } \\
\hline & gar nicht & gewöhnlich & erweitert \\
\hline $\begin{array}{c}\text { Der absolute } \\
\text { Differentialquotient } \\
\text { existiere }\end{array}$ & gar nicht & $*$ & $(3)(C)$ & $(4)(F)$ \\
\hline
\end{tabular}

Naturlich folgt aus der Existenz von (5) die von (I) und (3), aus der von (2) die von ( 1 ), aus der von ( 4 ) die von (3), schliesslich aus der von (6) die von (I) bis ( 5$)$; allgemein begreitt jede der obigen neun Voraussetzungen die daruber-, die links danebenstehenden und dic gleichzeitig distruber und weiter links stehenden V'oraussetzungen in sich.

Das erste Feld enthält überhaupt keine Voraussetzungen, braucht also nicht weiter betrachtet zu werden. Im ersten Teile dieser Arbeit will ich die Existenz der ubrigen acht Limites an einem Punkte betrachten; ein System geeigneter Beispiele wird zeigen, dass man aus der Existenz keines dieser ach Linites auf die Existenz eines in Schema weiter rechts oder weiter unten stehenden Limes schliessen kann. Ueberraschend erscheint dies Resultat besonders für die Voraussetzungen (2) und (4), denn ein unendlich kleines in der Nähe von $z$ gelegenes Dreieck wird im Falle (2) auf ein entsprechendes mit proportionalen Seiten, im Falle (4) auf ein entsprechendes mit gleichen Winkeln abgebildet. Man sollte also erwarten, duss man durch Anwendung des entsprechenden Aehnlichkeitssatzes der elementaren Geometrie im Falle (2) von der Streckentreue auf die Winkeltreue, im Falle $(+)$ von der Winkeltreue auf die Streckentreue schliessen kann. Tatsächlich ist dieser Schluss nicht zuliissig.

Im zweiten Teile dieser Arbeit, der bis auf wenige wiederbenutzte Gleichungen vom ersten Teile unabhängig ist, werde ich die Existenz derselben Limites an allen Stellen eines einfach zusammenhängenden, abgeschlossenen Gebietes voraussetzen. Für den Fall der Voraussetzung ( $j$ ) hat Herr Goursar ${ }^{\jmath}$ ) bewiesen, dass die Funktion eine analytische sein muss.

I) E. Goursat : a) Dimonstration du theorime de Cauchy [Acta Mathematica, Bd. IV (1884), S. $197-200$; b) Sur la difinition generale des fontions andylyiques d'apris CAuchr [Transactions of the American Mathematical Saciety, Bd. I (1900), S. 14-16]. - Ferner bethandeln den gleichen Gegenstand: c) E. H. Moore, A simple proof of the fundamental CauchY-Goursat theorem [Transactions of the American Muthematical Society, Bd. I (i90o), S. 499-j06]; d) A. Pringsheim, Ueber den Goursat' siben Beweis de's CtuChy'siben Integralsalies [Ibid., Bd II (1901), S. 413421];e) A. Pringsheim, Der 
Dasselbe will ich beweisen fur die V'oraussetzung (2) und mit einer gewissen Einschränkung fur dic Voraussetzung (4). Ich weiss nicht, ob der Satz auch ohne diese Einschränkung gilt. Ferner muss ich die Frage offen lassen, ob bereits aus der Voraussetzung (I) oder der Voraussetzung (3) an allen Punkten eines einfach zusammenhängenden, abgeschlossenen Gabietes folgt, dass die Funktion eine analytische sein muss.

\section{Erster Teil.}

Ich gebe nun für jede der acht Toraussetzungen des obigen Schemas Beispiele von Funktionen, die an einem Punkte genau dieser Voraussetzung und keiner weiteren des Schemas genügen. Die dem Schema beigefugten grossen lateinischen Buchstahen beziehen sich auf das zugehörige Beispiel.

Buispiel (t): Es bedeute $i$ die zu $i$ konjugierte Zahl. Es sei

$$
f(z)=\sum_{n=0}^{x}\left(\sum_{v=0}^{n} a_{v, n-v} z^{v} \bar{z}^{n-v}\right)
$$

Es konvergiere für eine reelle positive Zahl $r$

$$
\sum_{n=0}^{\kappa}\left(r^{n} \sum_{v=0}^{n}\left|a_{v, n-v}\right|\right)
$$

dann konvergiert $f(z)$, für alle $z$, für die $|z| \leq r$, absolut.

SATZ (I). - W'enn

$$
a_{0, \mathrm{1}}=\mathrm{o}
$$

so besität $f(\tilde{i})$ an der Sielle $z=0$ einen erweiterten Differentialquotienten, nämlich $a_{1,0}$. Es ist also zu zeigen, dass

$$
\lim _{\substack{z_{1}=0 \\ i_{2}=0}} \frac{f\left(z_{2}\right)-f\left(z_{1}\right)}{z_{2}-z_{1}}=a_{1,0} .
$$

Die Bezeichnung sei so gewählt, dass $\left|z_{2}\right| \gg\left|z_{1}\right|$. Es ist

$$
\frac{f\left(\tilde{i}_{2}\right)-f\left(\tilde{i}_{1}\right)}{\tilde{i}_{2}-z_{1}}=\frac{a_{0,0}-a_{0,0}}{\tilde{z}_{2}-\tilde{z}_{1}}+\frac{a_{1,0} z_{2}-a_{1,0} \tilde{z}_{1}}{z_{2}-z_{1}}+\sum_{i_{n=2}}^{\infty}\left(\sum_{\nu=0}^{n} a_{\nu, n-\nu} \cdot \frac{\tilde{z}_{2}^{\nu} \cdot \bar{z}_{2}^{n-\nu}-z_{1}^{\nu} \cdot \bar{z}_{1}^{n-\nu}}{z_{2}-z_{1}}\right)
$$

CAUCHY-Goursatsche Integralsal: und seine Utberiragung auf reelle Kurven-Integrale [Sitzungsberichte der m.thematisch-physikalischen Klasse der K. B. Akademie der Wissenschaften zu Munchen. Bd. XXXIII (1903), S. 67j-682]: f) L. HefFTer. Zur Theorie der reellen Kurvenintegrale [Nachrichten von der Kgl. Gesellschaft der Wissenschaften zu Gottingen, Mathematisch-physikalische Klasse. Jahrgang 1902, S. I I5-Ifo];g) L. Heffeter, Zum Beweis des Cauchy-Goursatschen Integralsatzes [lbid., Jahrgang 1903, S. 312-316]; h) L. HeffTeR, Über die von einem Integrationsu'eg von vornherein unabbängige Definition des bestimmten Integrals im iw'eidimensionalen Gebiet [Ibid., Jahrgang 1904, S. 196-200]. 
also

(9)

$$
\begin{aligned}
& \left|\frac{f\left(z_{2}\right)-f}{\left.z_{2}-z_{1}\right)}-a_{1,0}\right|=\left|\sum_{n=2}^{\infty}\left(\sum_{\nu=0}^{u} a_{v, n-v} \cdot \frac{z_{2}^{v} \cdot \bar{z}_{2}^{n-\nu}-z_{1}^{\nu} \cdot \bar{z}_{1}^{n-\nu}}{z_{2}-z_{1}}\right)\right| \\
& \leq \sum_{n=2}^{\infty}\left(\sum_{v=0}^{n}\left|a_{v, n-v}\right| \cdot\left|\frac{z_{2}^{v} \cdot \bar{i}_{2}^{n-\nu}-z_{1}^{\nu} \cdot \bar{i}_{1}^{n-\nu}}{z_{2}-z_{1}}\right|\right) \\
& =\sum_{n=2}^{\infty}\left(\sum_{\nu=0}^{n}\left|a_{v, n-\nu}\right| \cdot\left|\frac{\left(z_{2}^{\nu} \cdot \bar{z}_{2}^{n-\nu}-z_{1}^{\nu} \cdot \bar{z}_{2}^{n-\nu}\right)+\left(z_{1}^{\nu} \cdot \bar{z}_{2}^{n-\nu}-z_{1}^{\nu} \cdot \bar{z}_{1}^{n-\nu}\right)}{z_{2}-z_{1}}\right|\right) \\
& \leq \sum_{n=2}^{\infty}\left(\sum_{\nu=0}^{n}\left|a_{\nu, n-v}\right| \cdot\left\{\left|\frac{\bar{z}_{2}^{n-v} \cdot\left(z_{2}^{\nu}-z_{1}^{v}\right)}{z_{2}-\overline{z_{1}}}\right|+\left|\frac{\tilde{z}_{1}^{\nu} \cdot\left(\overline{z_{2}^{n-v}}-\bar{z}_{1}^{n-\nu}\right)}{\overline{z_{2}}-\bar{z}_{1}}\right|\right\}\right)
\end{aligned}
$$

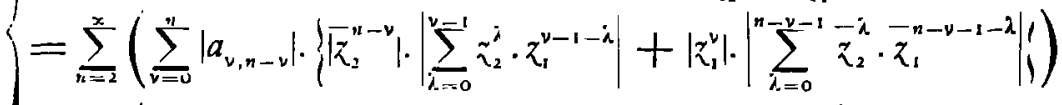

$$
\begin{aligned}
& \leq \sum_{n=2}^{\infty}\left(\sum_{\nu=0}^{n}\left|a_{\nu, n-\nu}\right| \cdot\left\{\left|\bar{z}_{2}\right|^{n-\nu} \cdot \sum_{\lambda=0}^{v}\left(\left|z_{2}\right|^{\lambda} \cdot\left|z_{1}\right|^{\nu-1-\lambda}\right)+\left|\tilde{z}_{1}^{\nu}\right| \cdot \sum_{i=0}^{n-v-1}\left(\left|\bar{z}_{2}\right|^{\lambda} \cdot\left|\bar{z}_{1}\right|^{n-\nu-1-\lambda}\right)_{j}^{\prime}\right)\right. \\
& \left.\leq \sum_{n=2}^{\infty}\left(\sum_{\nu=0}^{n-}\left|a_{v, n-\nu}\right| \cdot\left|z_{2}\right|^{n-\nu} \cdot \sum_{\lambda=0}^{v-1}\left|\tilde{z}_{2}\right|^{\lambda+\nu-1-\lambda}+\left|z_{2}\right|^{\nu} \cdot \sum_{\lambda=0}^{n-y-1}\left|z_{2}\right|^{\lambda+n-\nu-1-\lambda \mid}\right\}\right) \\
& =\sum_{n=2}^{\infty}\left(\sum_{v=0}^{n}\left|a_{v, n-v}\right| \cdot\left|z_{2}\right|^{n-1} \cdot(v+n-v)\right) \\
& =\sum_{n=2}^{\infty}\left(n\left|\tilde{n}_{2}\right|^{n-1} \cdot \sum_{\nu=0}^{n}\left|a_{v, n-\nu}\right|\right) \text {. }
\end{aligned}
$$

Der letzte Ausdruck konvergiert aber nach einem bekannten Satze aus der Theorie der Potenzreihen für $|z|<r$, weun ( 7 ) in demselben Gebiet konvergiert. Der letzte Ausdruck ist ferner von der Form $\left|z_{2}\right| \cdot \mathbb{P}\left|z_{2}\right|$, wo $\mathfrak{P}\left|z_{2}\right|$ eine nach steigenden Potenzen von $\left|z_{2}\right|$ entwickelte Potenzreihe mit positiven Koeffizienten bedeutet. Fúr alle

ist

$$
\left|z_{2}\right|<r^{\prime}<r
$$

$$
\mathfrak{B}\left|z_{2}\right|<\mathfrak{B}\left(r^{\prime}\right)=P \text {. }
$$

Hierin ist $P$ eine feste Zahl. Also ist, wenn $\left|z_{1}\right| \leq\left|z_{2}\right|$

$$
\left|\frac{f\left(z_{2}\right)-f\left(z_{1}\right)}{z_{2}-z_{1}}-a_{1,0}\right|<\left|z_{2}\right| \cdot P,
$$

kann also beliebig klein gemacht werden. Damit ist (8) bewiesen.

Aus dem Satz (I) folgt als spezieller Fall:

Eine analytische: Funktion besitzt an jeder Stelle, an der sie regulär ist, einen erweiterten Differentialquotienten.

Natürlich würde der Beweis dieser speziellen Behauptung einfacher ausfallen. Ich brauche aber den allgemeineren Satz (I) weiter unten.

Nachdem ich vorweg ein Beispiel für die umfassendste Voraussetzung (6) gegeben habe, gehe ich zu den geringsten Voraussetzungen (I) und (3) über, für die Beispiele leicht $z u$ bilden sind.

Beispiel $(B)$ : Es sei $f(z)=u$ definiert durch die Gleichungen

$$
\begin{aligned}
|u| & =|z|, \\
\operatorname{sgn} u & =1 \cdot \overline{\operatorname{sgn} z,}
\end{aligned}
$$


wobei das V'orzeichen der Quadratwurzel so zu wählen ist, dass

und speziell

$$
\Im(\sqrt{\operatorname{sgn} z}) \supseteq 0
$$

$$
\mathbf{V}^{\prime} \overline{\mathrm{I}}=\mathrm{I}
$$

zu setzen ist. (Es sei stets, wenn $a+b i$ ein komplexe Zahl, der Realteil

der Imaginärteil

$$
\begin{aligned}
& \Re(a+b i)=a, \\
& \mathfrak{J}(a+b i)=b) .
\end{aligned}
$$

An Punkte $\tau=0$ existiert der gewöhnliche absolute Differentialquotient. Es ist

$$
\lim _{i=0}\left|\frac{u-0}{z-0}\right|=\lim _{i=0} \frac{|u|}{|z|}=\lim _{i=0} \mathrm{I}=\mathrm{I} .
$$

Dagegen existiert kein erweiterter absoluter Differentialquotient. Es sei $i_{2}$ reell und positiv und

dann ist

$$
z_{1}=-\tau_{2}
$$

$$
u_{2}=z_{2} \text {, }
$$

Also wird

$$
u_{1}=i \bar{z}_{1} \mid=i z_{2} \text {. }
$$

$$
\left|\frac{u_{2}-u_{1}}{i_{2}-i_{1}}\right|=\left|\frac{z_{2}-i i_{2}}{i_{2}+z_{2}}\right|=\left|\frac{\mathrm{I}-i}{2}\right|=\frac{1^{\prime} \frac{}{2}}{2} \neq \mathrm{I} .
$$

Ferner existiert kein Signum-Differentialquotient. Es sei

dann ist

$$
\tilde{z}_{2}=2 z_{1}
$$

also

$$
u_{2}=2 u_{1}=2\left|z_{1}\right| 1^{\prime} \overline{\operatorname{sgn} z_{1}} \text {, }
$$

$$
\operatorname{sgn} \frac{u_{2}-u_{1}}{\tilde{i}_{2}-\tilde{i}_{1}}=\operatorname{sgn} \frac{\tilde{i}_{1} \mid l \overline{\operatorname{sgn} \tilde{i}_{1}}}{\tilde{i}_{1}}=\frac{1^{\prime} \operatorname{sgn} \tilde{i}_{1}}{\operatorname{sgn} \tilde{i}_{1}}=\frac{1}{1 \operatorname{sgn} \tilde{z}_{1}} .
$$

Das Signum des Differenzenquotienten kanı also jeden Wert auf dem Einheitskreise mit negativem Imaginärteil annehmen.

Beispiel $(C)$ : Es sei $f(z)=u$ definiert durch die Gleichungen

$$
\begin{gathered}
|u|=1 \cdot \overline{|z|}, \\
\operatorname{sgn} u=\operatorname{sgn} z .
\end{gathered}
$$

Es existiert im Punkte $\tilde{\imath}=0$ der gewöhnliche Signum-Differentialquotient. Es ist

$$
\lim _{i=0} \operatorname{sgn} \frac{u-0}{i-0}=\lim _{i=0} \frac{\operatorname{sgn} u}{\operatorname{sgn} z}=\lim _{i=0} \mathrm{I}=\mathrm{I} \text {. }
$$

D.gegen existiert kein erweiterter Signum-Differentialquotient. Es sei

also wird

$$
z_{2}=2 i z_{1},
$$

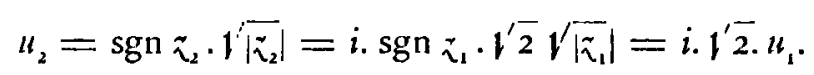


Es ergibt sich

$$
\begin{aligned}
& \operatorname{sgn} \frac{u_{2}-u_{1}}{z_{2}-z_{1}}=\operatorname{sgn}\left(\frac{i \sqrt{2}-1}{2 i-\mathrm{I}} \cdot \frac{u_{1}}{z_{1}}\right)=\operatorname{sgn} \frac{i 1^{\prime}-\overline{2}-\mathrm{I}}{2 i-\mathrm{I}} \cdot \frac{\operatorname{sgn} u_{1}}{\operatorname{sgn} z_{1}}
\end{aligned}
$$

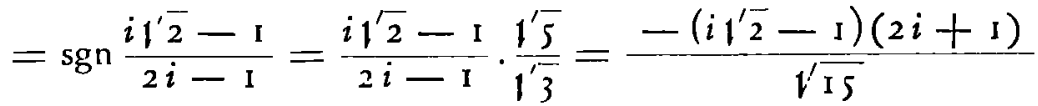

$$
\begin{aligned}
& =\frac{21^{\prime}+1+i\left(2-1^{\prime} \frac{1}{2}\right)}{1 \sqrt{15}} \neq \mathrm{I} \text {. }
\end{aligned}
$$

Ebenso existiert kein gewöhnlicher absoluter Differentialquotient. Es ist

$$
\lim _{i=0}\left|\frac{u-0}{z-0}\right|=\lim _{i=0} \frac{|u|}{|\tilde{i}|}=\lim _{i=0} \frac{1}{1^{\mid}|\overline{\mid}|}=\infty \text {. }
$$

Bevor ich in der Reihe der Beispiele fortfahre, beweise ich die Ungleichbeitsbeziehung

$$
\left|e^{i 2}-1\right|<x
$$

Hierin sei $x$ eine reelle positive Zahl.

Wenn $x>2$, so ist

$$
\left|e^{i x}-1\right| \leq\left|e^{i x}\right|+1=2<x .
$$

Es sei also $x \leq 2$. Dann ist

$$
\begin{gathered}
\left|e^{i x}-\mathrm{I}\right|^{2}=\left(e^{i x}-\mathrm{I}\right)\left(e^{-i x}-\mathrm{I}\right)=2-e^{i x}-e^{-i x} \\
=2-2\left(x-\frac{x^{2}}{2 !}+\frac{x^{4}}{4 !}-\cdots\right)=2\left(\frac{x^{2}}{2 !}-\frac{x^{4}}{4 !}+\cdots\right) .
\end{gathered}
$$

Es ist für $n \supseteq \mathrm{I}$

weil

$$
\frac{x^{2 n}}{(2 n) !}>\frac{x^{2 n+2}}{(2 n+2) !}
$$

$$
\frac{x^{2}}{(2 n+1)(2 n+2)}<\frac{4}{12}=\frac{1}{3}<1 \text {. }
$$

Also ist die obige Reihe alternierend mit ständig abnehmenden Gliedern. Es ist also

oder

$$
\left|e^{i x}-\mathrm{I}\right|^{2}<x^{2}
$$

was zu beweisen war.

$$
\left|e^{x x}-\mathrm{I}\right|<x \text {, }
$$

Beispiel (D): Es sei

wo

und es sei definiert

$$
\begin{gathered}
z=r \cdot e^{\imath j} \\
r=|z|,
\end{gathered}
$$

$$
f(z)=u=z+r^{2} \cdot e^{i j \cdot r^{-1}}
$$

Für a werde der Hauptwert gesetzt:

$$
0 \leq a<2 \pi .
$$




\section{Ferner sei}

$$
f(0)=0 .
$$

Die Funktion ist in allen Punkten der positiven reellen Axe ausser im Nullpunkte und anderer Ausnahmepunkten unstetig.

Es besitzt $f(i)$ im Punkte $z=0$ zwar cinen gewöhnlichen Differentialquotienten, aber weder einen erweiterten absoluten noch einen erweiterten Signum-Differentialquotienten.

Es ist

also kann

$$
\frac{u-0}{z-0}=1+\frac{r^{2}}{z} \cdot e^{i 3 \cdot r-1}
$$

$$
\left|\frac{u}{z}-\mathrm{I}\right|=\left|\frac{r^{2}}{z} \cdot e^{13} r^{-1}\right|=r
$$

beliebig klein gemacht werden. Es ist somit

$$
\lim _{i=0} \frac{u}{z}=\mathrm{I} \text {. }
$$

Man bilde den erweiterten Differenzenquotienten und setze speziell $r_{2}=r_{1}=r$ :

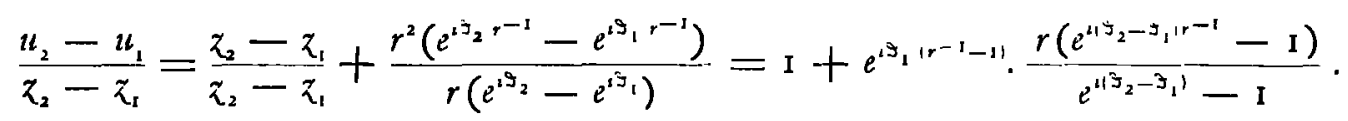

Man setze nun speziell

dann wird

$$
\nabla_{2}-\Im_{1}=\pi r
$$

$$
\frac{u_{2}-u_{1}}{z_{2}-z_{1}}=\mathrm{I}+e^{\left.i \vartheta_{1}{ }^{\prime r-1}-1\right)} \cdot \frac{r\left(e^{i \pi}-\mathrm{I}\right)}{e^{i \pi r}-\mathrm{I}} \text {. }
$$

Nun erhält man unter Benutzung der Ungleichheitsbeziehung (Io)

$$
|A(r)| \equiv\left|\frac{r\left(e^{i \pi}-1\right)}{e^{i \pi r}-1}\right|>\frac{2 r}{\pi r}=\frac{2}{\pi} .
$$

Das gilt für beliebig kleine $r$. Der Ausdruck $A(r)$ ist bei Festhaltung der durch die Gleichung (II) bestimmten Difierenz $z_{2}-J_{1}$ von $z_{1}$ nicht abhängig. Also kann man über $\sigma_{1}$ bei beliebig kleinem festem $r$ noch so verfügen, dass $e^{\left.i j_{1} r^{-1}-1\right)}$ jeden beliebigen Wert $\sigma$ auf den Einheitskreise annimmt. Man erhält also

$$
\frac{u_{2}-u_{1}}{z_{2}-z_{1}}=1+\sigma \cdot A(r) \text {. }
$$

Man kann z. B. über o so verfügen, dass s. $A(r)$ reell und positiv wird, dann wird nach (12)

$$
|\mathrm{I}+\sigma \cdot A(r)|>\mathrm{I}+\frac{2}{\pi}>\mathrm{I} \text {. }
$$


Also existiert kein erweiterter absoluter Differentialquotient. Man kann ferner uber $\sigma$ so verfügen, dass $\sigma . A(r)$ rein positiv imaginär wird; dann wird

$$
\operatorname{sgn}(\mathrm{I}+\sigma \cdot A(r))=\operatorname{sgn}(\mathrm{I}+i|A(r)|) \neq \mathrm{I} .
$$

Also evistiert auch kein erweiterter Signum-Differentialquotient.

Für das Folgende brauche ich noch eine weitere Ungleichheitsbeziehung. Es sei $x$ eine komplexe Zahl, $|x|<\mathrm{I}$. Dann ist

(13) $|\log (\mathrm{I}+x)|=\left|\sum_{n=1}^{\infty}(-\mathrm{I})^{n-1} \cdot \frac{x^{n}}{n}\right| \leq \sum_{n=1}^{\infty} \frac{|x|^{n}}{n}=\log \left(\frac{\mathrm{I}}{\mathrm{I}-|x|}\right)<\sum_{n=1}^{\infty}|x|^{n}=\frac{|x|}{\mathrm{I}-|x|}=\frac{\mathrm{I}}{\frac{\mathrm{I}}{|x|}-\mathrm{I}}$.

Beispiel $(E)$ : Es sei für das Gebiet $|\mathfrak{z}|<e^{-1}$ die Funktion $f(\mathfrak{z})=u$ definiert durch die Gleichungen

$$
\begin{aligned}
& f(z)=u=z \cdot e^{u \cdot \log \log |i|^{-1}}, \\
& f(0)=0 .
\end{aligned}
$$

Die Einführung der reellen positiven Konstanten $c$ in Exponenten erscheint an dieser Stelle überflüssig, ist aber für spätere $Z$ wecke nützlich.

An der Stelle $z=0$ besitzt $f(z)$ einen erweiterten absoluten Differentialquotienten, aber keinen Signum-Differentialquotienten.

Zunächst ist

$$
\lim _{i=0}\left|\frac{u-0}{z-0}\right|=\lim _{i=0} \mathrm{I}=\mathrm{I} .
$$

Es soll ferner gezeigt werden, dass

$$
\lim _{\substack{i=0 \\ z_{2}=0}}\left|\frac{u_{2}-u_{1}}{z_{2}-\frac{u_{1}}{z_{1}}}\right|=\mathbf{1}
$$

Man wähle die Bezeichnung so, dass

$$
\left|z_{2}\right| \supseteq\left|z_{1}\right|
$$

Der Fall $\tilde{i}_{1}=0$ ist bereits erledigt. Man nehne also $\left|z_{1}\right|>0$ an. Es soll nach Annahme einer beliebig kleinen reellen positiven Zahl $\varepsilon$ eine hinreichend kleine reelle positive Zahl $\delta$ so bestimmt werden, dass, wenn

$$
\left|\tilde{z}_{1}\right| \leq\left|z_{2}\right|<\delta
$$

ist,

$$
\left|\frac{u_{2}-u_{1}}{z_{2}-z_{1}}\right|-\mathrm{I} \mid<\varepsilon .
$$


wird. Man erhält unter Benutzung von (ro)

$$
\begin{aligned}
& || \frac{u_{2}-u_{1}}{z_{2}-z_{1}}|-\mathrm{I}|=\left|\frac{z_{1} \cdot e^{u_{1} \log \log \left|z_{2}\right|^{-1}}-z_{1} \cdot e^{u^{\log \log \mid i_{1} 1^{-1}}}}{z_{2}-z_{1}}\right|-\mathrm{I} \mid
\end{aligned}
$$

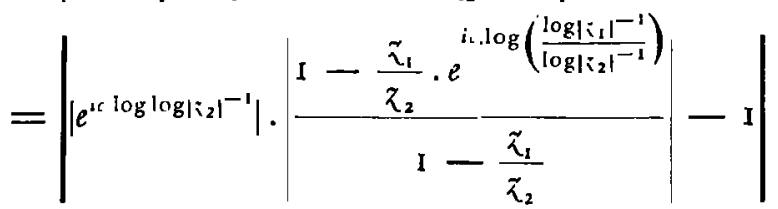

$$
\begin{aligned}
& \leq\left|\frac{1-\frac{z_{1}}{z_{2}} \cdot e^{i_{1} \cdot \log \left(\frac{\log \left|i_{i}\right|^{-1}}{\log \left|i_{2}\right|^{-1}}\right)}}{1-\frac{z_{1}}{z_{2}}}-1\right| \\
& =\mid \frac{\frac{z_{1}}{z_{2}}}{I-\frac{z_{1}}{z_{2}}}\left(1-e^{\left.i \cdot \log \left(\frac{\left.\log \right|_{1} I_{1}^{-1}}{\left.\left.\log \right|_{2}\right|^{-1}}\right)\right)} \mid\right. \\
& <\frac{\left|\frac{z_{1}}{z_{2}}\right|}{\left|1-\frac{z_{1}}{z_{2}}\right|} c \cdot \log \left(\frac{\log \left|z_{1}\right|^{-1}}{\log \left|z_{2}\right|^{-1}}\right) \\
& =\frac{\text { c. }\left|\frac{z_{1}}{z_{2}}\right|}{\left|1-\frac{z_{1}}{z_{2}}\right|} \cdot \log \left(1+\frac{\log \left|\frac{z_{1}}{z_{2}}\right|^{-1}}{\log \left|z_{2}\right|^{-1}}\right) \\
& <\frac{c \cdot\left|\frac{z_{1}}{z_{2}}\right| \cdot \log \left|\frac{z_{1}}{z_{2}}\right|^{-1}}{\left|1-\frac{z_{1}}{z_{2}}\right| \cdot \log \left|z_{2}\right|^{-1}},
\end{aligned}
$$

weil fur alle reellen $x>0$

Setzt man

$$
\log (1+\alpha)<\alpha .
$$

so ist

$$
\begin{aligned}
& \mathrm{I}-\frac{z_{1}}{z_{2}}=x, \\
& I-\left|\frac{z_{1}}{z_{2}}\right|=y,
\end{aligned}
$$

( 17 )

$$
y \leq|x| \text {. }
$$

Ferner ergibt sich aus (1 3 )

$$
\log \left|\frac{z_{1}}{z_{2}}\right|^{-1}=\log \left(\frac{I}{I-y}\right)<\frac{y}{I-y} .
$$


Setzt man (18) in (I6) ein, so erhält man unter Benutzung von (I7)

$$
|| \frac{u_{2}-u_{1}}{z_{2}-z_{1}}|-\mathrm{I}|<\frac{c y(1-y)}{(1-y)|x| \log \left|z_{2}\right|^{-1}} \leq \frac{c}{\log \left|z_{2}\right|^{-1}}<\varepsilon
$$

sowie

oder

$$
\log \left|z_{2}\right|^{-1}>\frac{c}{\varepsilon}
$$

$$
\left|z_{1}\right| \leq\left|z_{2}\right|<e^{-\frac{1}{\varepsilon}}=\delta
$$

Damit ist (I 5 ) oder (14) erfüllt, also die Existenz des erweiterten absoluten Differentialquotienten im Punkte $z=0$ bewiesen. Ein Signum-Differentialquorient existiert nicht. Denn

$$
\operatorname{sgn}\left(\frac{u-o}{z-0}\right)=\operatorname{sgn} e^{u \log \log |i|^{-1}}
$$

hat für $z=0$ keinen Limes.

Beispiel $(F)$ : Es sei fur das Gebiet $|z|<\imath^{-1}$ die Funktion $f(z)=u$ definiert durch die Gleichungen

$$
\begin{aligned}
& f(x)=u=\tilde{i} \log |z|^{-1}, \\
& f(0)=0 .
\end{aligned}
$$

An der Stelle $z=0$ besitzt $f(z)$ einen erweiterten Signum-Differentialquotienten, aber keinen absoluten Differentialquotienten.

Es ist

$$
\lim _{i=0} \operatorname{sgn} \frac{u-0}{z-0}=\lim _{i=0} \operatorname{sgn} \log ||^{-\mathrm{s}}=\lim _{i=0} \mathrm{I}=\mathrm{I} .
$$

Es soll gezeigt werden, dass

$$
\lim _{\substack{\gamma_{1}=0 \\ z_{2}=0}} \operatorname{sgn} \frac{u_{2}-u_{1}}{z_{2}-z_{1}}=\mathrm{I}
$$

Man wähle die Bezeichnung so, dass

$$
\left|z_{1}\right| \leqslant\left|z_{2}\right|
$$

Der Fall $z_{1}=0$ ist bereits erledigt. Man nehme also $\left|z_{1}\right|>0$ an. Es soll nach Annahme einer beliebig kleinen reellen positiven Zahl $\varepsilon$ eine hinreichend kleine reelle positive Zahl $\delta$ so bestimmt werden, dass, wenn

ist,

$$
\left|z_{1}\right| \leq\left|z_{2}\right|<\delta
$$

$$
\left|\operatorname{sgn} \frac{u_{2}-u_{1}}{z_{2}-z_{1}}-1\right|<\varepsilon
$$


wird. Es ist

(22)

$$
\begin{aligned}
& \left|\operatorname{sgn} \frac{u_{2}-u_{1}}{z_{2}-z_{1}}-1\right|=\left|\operatorname{sgn} \frac{z_{2} \cdot \log \left|z_{2}\right|^{-1}-z_{1} \cdot \log \left|z_{1}\right|^{-1}}{z_{2}-z_{1}}-1\right| \\
& =\left|\operatorname{sgn} \frac{z_{2} \log \left|z_{2}\right|^{-1}}{z_{2}} \cdot \operatorname{sgn} \frac{1-\frac{\tilde{z}_{1}}{z_{2}} \cdot \frac{\log \left|z_{1}\right|^{-1}}{\log \left|z_{2}\right|^{-1}}}{1-\frac{z_{1}}{z_{2}}}-I\right| \\
& =\left|\operatorname{sgn} \frac{1-\frac{z_{1}}{z_{2}}\left(1+\frac{\log \left|\frac{z_{1}}{z_{2}}\right|^{-1}}{\log \left|z_{2}\right|^{-1}}\right)}{1-\frac{z_{1}}{z_{2}}}-1\right| \\
& =\left|\operatorname{sgn}\left(1-\frac{\frac{z_{1}}{z_{2}} \cdot \log \left|\frac{z_{1}}{z_{2}}\right|^{-1}}{\left(1-\frac{z_{1}}{z_{2}}\right) \log \left|z_{2}\right|^{-1}}\right)-1\right| .
\end{aligned}
$$

Es sei $a$ eine komplexe Zahl, die der Bedingung $|a|<\mathrm{I}$ genügt. $\bar{a}$ bedeute die zu $a$ konjugierte Zahl. Es ist

also

$$
0 \leqslant\left(|a|^{2}+\Re(a)\right)^{2}=\Re^{2}(a)+2 \Re(a) \cdot|a|^{2}+|a|^{4}
$$

oder

$$
\left(1-|a|^{2}\right) \mathfrak{J}^{2}(a) \leq|a|^{2}+2|a|^{2} \cdot \Re(a)+|a|^{2} \Re^{2}(a)
$$

$$
\begin{aligned}
& \left(\frac{\Im(a)}{\mathrm{I}+\mathfrak{R}(a)}\right)^{2} \leq \frac{|a|^{2}}{\mathrm{I}-|a|^{2}}, \\
& \frac{\mathfrak{R}^{2}(1+a)+\mathfrak{I}^{2}(1+a)}{\Re^{2}(1+a)} \leq \frac{1}{1-|a|^{2}}, \\
& \frac{\Re(\mathrm{I}+a)}{|\mathrm{I}+a|} \geq 1 / \overline{1-|a|^{2}}, \\
& 2-2 \Re(\operatorname{sgn}(1+a)) \leq 2-2 \gamma^{\prime} \overline{\mathrm{I}-|a|^{2}},
\end{aligned}
$$

$\operatorname{sgn}(1+a) \cdot \operatorname{sgn}(1+\bar{a})-\operatorname{sgn}(1+a)-\operatorname{sgn}(1+\bar{a})+1 \leq 2-2 \sqrt{1-|a|^{2}}$

Man setze

ferner bestinume man

$$
|\operatorname{sgn}(1+a)-\mathrm{I}| \leq \sqrt{2-2 \sqrt{1-|a|^{2}}} \text {. }
$$

$$
a=-\frac{\frac{z_{1}}{z_{2}} \cdot \log \left|\frac{z_{1}}{z_{2}}\right|^{-1}}{\left(1-\frac{z_{1}}{\tilde{z}_{2}}\right) \log \left|z_{2}\right|^{-1}}
$$

$$
\left|z_{1}\right| \leq\left|z_{2}\right|<e^{\frac{-1}{11^{1 / 1-\frac{e^{2}}{4}}}}=\delta .
$$


Dann ist

(24)

und nach (16), (19) und (24)

$$
\log \left|z_{2}\right|^{-1}>\frac{I}{\varepsilon \sqrt{I-\frac{\varepsilon^{2}}{4}}}
$$

$$
|a|=\frac{\left|\frac{z_{1}}{z_{2}}\right| \cdot \log \left|\frac{z_{1}}{z_{2}}\right|^{-1}}{\left|1-\frac{z_{1}}{z_{2}}\right| \log \left|z_{2}\right|^{-1}} \leq \frac{1}{\log \left|z_{2}\right|^{-1}}<\varepsilon \sqrt{I-\frac{\varepsilon^{2}}{4}} ;
$$

also ist nach (22), (23) und (25)

(26)

$$
\begin{aligned}
& || \operatorname{sgn} \frac{u_{2}-u_{1}}{z_{2}-z_{1}}-1|=| \operatorname{sgn}(\mathrm{I}+a)-\mathrm{I} \mid \\
& \left\langle\leq 1 \sqrt{2-2 \sqrt{1-|a|^{2}}}<\sqrt{2-2 \sqrt{1-\varepsilon^{2}\left(I-\frac{\varepsilon^{2}}{f}\right)}}=\sqrt{2-2\left(I-\frac{\varepsilon^{2}}{2}\right)}=\varepsilon .\right.
\end{aligned}
$$

Damit ist (2 I) erfüllt, also gilt auch (20).

Es existiert aber kcin absoluter Differentialquotient. Es ist

$$
\lim _{i=0}\left|\frac{u-o}{z-o}\right|=\lim _{i=0} \log |z|^{-1}=\infty \text {. }
$$

Die beiden folgenden Beispiele werden aus den beiden vorhergehenden durch Häufung gebildet.

Beispiel $(G)$ : Es seien drei monoton gegen o abnehmende Reihen reeller positiver Zahlen gegeben

$$
\lim _{n=\infty} a_{n}=0, \quad \lim _{n=\infty} c_{n}=0, \quad \lim _{n \rightarrow \infty} p_{n}=0,
$$

die den Bedingungen genügen

(27)

(30)

Es konvergiere die Reihe

$$
\begin{gathered}
a_{n}<e^{-2}, \\
c_{n}<\mathrm{I}, \\
p_{n}<\frac{a_{n-1}-a_{n}}{2}, \\
\rho_{n}<\frac{a_{n}-a_{n+1}}{2} .
\end{gathered}
$$

$$
\sum_{n=1}^{\infty} c_{n} \cdot \log \log p_{n}^{-1}=C .
$$

Aus (3r) folgt die Konvergenz der Reihe

ebenso konvergiert

$$
\sum_{n=1}^{\infty} c_{n} \cdot \log \log a_{n}^{-1}=B,
$$

$$
\sum_{n=1}^{\infty} \frac{2 c_{n}}{\log a_{n}^{-1}}=A
$$

Es sei nun $f(z)=u$ definiert durch die Gleichung

$$
f(z)=u=(1+A i) z+\sum_{n=1}^{\infty} g_{n}(z)
$$


hierin ist

hierin wieder

$$
g_{n}(z)=\sum_{\psi=0}^{3} h_{n \psi}(z)
$$

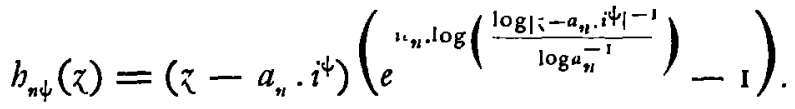

Es ist $h_{n \psi}(0)=0$, also $g_{n}(0)=0, f(0)=0$.

Es soll zunächst gezeigt werden, dass $f(\tau)$ an der Stelle $z=0$ den gewöhnlichen Differentialquotienten I besitzt; die $\rho_{n}$ benutze ich dabei noch nicht. Es soll also gezeigt werden, dass

$$
\lim _{:=0}\left(\frac{f(\pi)}{z}-1\right)=0
$$

d. h. nach Annahme einer beliebig kleinen reellen positiven Grösse $\varepsilon$ soll ein hinreichend kleine reelle positive Grösse $\delta$ so bestimmt werden, dass, wenn

ist,

$$
|z|<\delta
$$

(35) $\left|\frac{f(\tilde{z})}{z}-1\right|=\left|\sum_{n=1}^{\infty} \frac{g_{n}(z)}{z}+(\mathrm{I}+A i)-\mathrm{I}\right|=\left|\sum_{n=1}^{\infty}\left(\frac{g_{n}(\tilde{z})}{z}+\frac{2 i c_{n}}{\log a_{n}^{-1}}\right)\right|<\varepsilon$

wird. Es mögen $M \supseteq N$ zwei noch zu bestimmende positive ganze Zahlen sein. Dann ist

$$
\left\{\begin{array}{c}
\left|\sum_{n=1}^{\infty}\left(\frac{g_{n}(\tilde{z})}{z}+\frac{2 i c_{n}}{\log a_{n}^{-1}}\right)\right| \leq \sum_{n=1}^{N}\left|\frac{g_{n}(z)}{z}+\frac{2 i c_{n}}{\log a_{n}^{-1}}\right|+\sum_{n=1}^{M}\left|\frac{g_{n}(z)}{z}+\frac{2 i c_{n}}{\log a_{n}^{-1}}\right| \\
+\sum_{n=M+1}^{\infty}\left|\frac{g_{n}(\tilde{I})}{z}\right|+\sum_{n=M+1}^{\infty} \frac{2 c_{n}}{\log a_{n}^{-1}}=S_{1}+S_{2}+S_{3}+S_{1}
\end{array}\right.
$$

Hierin sei zunächst $N$ so bestimmt, dass

$$
\begin{gathered}
\sum_{n=N^{\prime}+1}^{\infty} \frac{2 c_{n}}{\log a_{n}^{-1}}<\frac{7 \varepsilon}{20 \mathrm{I}}, \\
\sum_{n=N^{\prime}+1}^{\infty} c_{n} \log \log a_{n}^{-1}<\frac{\varepsilon}{3^{6}}, \\
\delta<a_{N^{\prime}} \sqrt{\frac{7 \varepsilon}{720 A}}
\end{gathered}
$$

sodann bestimme man

und

(40)

$$
\delta<\frac{a_{N}}{2}
$$

Für das vorliegende z, das absolut kleiner als $\delta$ sei, bestimne man $M$ so gross, dass

$$
\frac{a_{M+1}}{2}<|z| \leq \frac{a_{M}}{2}
$$

Es genügt nach (40) $N$ der zweiten dieser Bedingungen, es kann auch $N=M$ sein, so dass $S_{2}=0$ wird. 
Zur Abschätzung von $S_{1}$ und $S_{2}$ verfahre man folgendermassen. Es ist nach (33)

Wenn

$$
b_{n \downarrow}(i)=a_{n} \cdot i^{\psi}\left(\frac{z}{a_{n} \cdot i^{\psi}}-1\right)\left[e^{n_{n} \log \left(1-\frac{\log \left(1-\frac{i}{a_{n}, \psi}\right)+\log \left(1-\frac{\bar{\vdots}}{a_{n} \cdot 1-i \psi}\right)}{2 \log a_{n}^{-1}}\right)}-\mathrm{I}\right] \text {. }
$$

oder

$$
\mathrm{I}-\frac{|z|}{a_{n}}>a_{n}
$$

(44)

$$
|i|<a_{n}-a_{n}^{2}
$$

so lassen sich die inneren und äusseren Logarithmen im Exponenten von $e$ auf der rechten Seite von $\left(4^{2}\right)$ in konvergente Potenzreihen entwickeln, also auch die Exponentialfunktion selber. Es ist für $S_{1}$ und $S_{2}$ nach $(+\mathrm{I})$

Darum ist wegen $(27)$

$$
|z| \leqslant \frac{a_{n}}{2}
$$

$$
\mathrm{I}-\frac{|z|}{a_{n}} \geqslant \frac{\mathrm{I}}{2}>e^{-2}>a_{n}
$$

also (43) oder (44) erfüllt. Man erhält also

Also wird

$$
\begin{aligned}
b_{n \psi}(z) & =a_{n} \cdot i \psi \cdot k_{n}\left(\frac{z}{a_{n} \cdot i^{\psi}}, \frac{\bar{z}}{a_{n} \cdot i^{-\psi}}\right) \\
& =a_{n} \cdot i^{\psi} \cdot \sum_{r=0}^{\infty}\left(\sum_{\rho=0}^{r} b_{\rho, r-\rho} \cdot \frac{z^{\rho} \bar{z}^{r-\rho}}{a_{n}^{r} \cdot i^{i(2 \rho-r)}}\right) .
\end{aligned}
$$

Hierin ist

$$
g_{n}(z)=a_{n} \sum_{r=0}^{r}\left(\sum_{\rho=0}^{r}\left(b_{\rho, r-p} \cdot \frac{z^{r\left(z^{r-\rho}\right.}}{a_{n}^{r}} \cdot \sum_{\psi=0}^{3} i^{i(r-2 i p+1)}\right)\right) \text {. }
$$

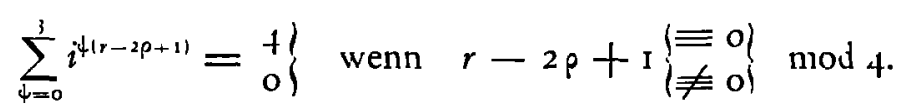

Die Summe ist also nur dann von o verschieden, wenn

$$
\begin{aligned}
& r=2 s-\mathrm{I}, \\
& \mathrm{p} \equiv s \bmod 2 .
\end{aligned}
$$

Für $r=\mathrm{I}$ ist $s=\mathrm{I}$, also auch $\rho=\mathrm{I}$. Setzt man

so ist

$$
g_{n}(z)=\sum_{r=0}^{\infty}\left(\sum_{\rho=0}^{r} c_{\rho, r-\rho} \cdot z^{\rho} \vec{z}^{r-\rho}\right),
$$

(48)

$$
c_{8,0}={ }_{4} b_{1,0}
$$

$$
c_{0,1}=0 \text {. }
$$


Also kesitzt $g_{n}(z)$ nach Satz (I) an der Stelle $z=0$ einen erweiterten Differentialquotienten.

Man erhält eine Majorante von $g_{n}(z)$ d. h. eine Potenzentwicklung nach $z$ und $\bar{z}$, in der jeder Koeffizient positiv und absolut grösser ist als der entsprechende in $g_{n}(z)$, wenn man eine Majorante von ${ }_{4} b_{n \downarrow}(z)$ bildet und in dieser die Glieder, deren Indices nicht (46) und (47) gleichzeitig genügen, nach Belieben ganz oder teilweise fortlässt, z. B. nur die Glieder mit ungeradem $r$ berücksichtigt.

Es ist

$$
e^{\left.i i_{n} \cdot \log \mid 1+y\right)}=(\mathrm{I}+y)^{i i_{1}}
$$

Die Binomialentwicklung der rechten Seite konvergiert bekanntlich, so lange $|y|<1$. Es ist für $r \gg I$ der Binomialkoeffizient

$$
\left(\begin{array}{c}
i c_{n} \\
r
\end{array}\right)=\frac{i c_{n}}{r} \cdot \prod_{\nu=1}^{r-1}\left(-\mathrm{I}+\frac{i c_{n}}{\nu}\right) .
$$

Also erbält man unter Benutzung der bekannten Formel

die Abschätzung

$$
\sin \left(i c_{n} \pi\right)=i c_{n} \pi \prod_{\nu=1}^{x}\left(1+\frac{c_{n}^{2}}{v^{2}}\right)
$$

$$
\begin{aligned}
& \left|\left(\begin{array}{c}
i c_{n} \\
r
\end{array}\right)\right|=\frac{c_{n}}{r} \cdot \sqrt{\prod_{v=1}^{r-1}\left(\mathrm{I}+\frac{c_{n}^{2}}{v^{2}}\right)}<\frac{i_{n}}{r} \cdot 1 \sqrt{\prod_{v=1}^{\infty}\left(\mathrm{I}+\frac{c_{n}^{2}}{v^{2}}\right)} \\
= & \frac{c_{n}}{r} \cdot \sqrt{\frac{\sin \left(i c_{n} \pi\right)}{i c_{n} \pi}}=\frac{c_{n}}{r} \cdot \sqrt{\frac{e^{-i_{n} \pi}-e^{c_{n} \pi}}{2 i\left(i c_{n} \pi\right)}}=\frac{c_{n}}{r} \cdot \sqrt{\sum_{\mu=0}^{\infty} \frac{\left(c_{n} \pi\right)^{2 \mu}}{(2 \mu+\mathrm{J}) !}} .
\end{aligned}
$$

Der Radikandus des letzten Ausdrucks ist eine monoton wachsende Funktion von $c_{n}$. Also ist nach (28)

$$
\frac{c_{n}}{r} \cdot \sqrt{\sum_{\mu=0} \frac{\left(c_{n} \pi\right)^{2 \mu}}{(2 \mu+\mathrm{I}) !}}<\frac{c_{n}}{r} \cdot \sqrt{\frac{e^{\pi}-e^{-\pi}}{2 \pi}}<\frac{2 c_{n}}{r} .
$$

Es ist also

$$
\left\{\begin{array}{c}
\left|b_{n \psi}(\tilde{z})\right|=a_{n} \cdot\left|k_{n}\left(\frac{z}{a_{n} \cdot i^{\psi}}, \frac{\bar{z}}{a_{n} \cdot i^{-\psi}}\right)\right| \\
<a_{n} \cdot\left(\mathrm{I}+\frac{|z|}{a_{n}}\right) \cdot 2 c_{n} \cdot \sum_{i=1}^{\infty} \frac{\mathrm{I}}{r} \cdot\left(\frac{\sum_{s=1}^{\infty} \frac{\mathrm{I}}{s}\left(\frac{|z|}{a_{n}}\right)^{s}}{\log a_{n}^{-1}}\right)^{r} \\
<a_{n} \cdot\left(\mathrm{I}+\frac{|z|}{a_{n}}\right) \cdot 2 c_{n} \cdot \sum_{r=1}^{\infty}\left(\frac{\sum_{j=1}^{\infty}\left(\frac{|z|}{a_{n}}\right)^{s}}{\log a_{n}^{-1}}\right)^{r}
\end{array}\right.
$$

Die innere und die äussere unendliche Reihe in diesem Ausdrucke konvergieren wegen 
(45). Es ist also

(5I)

$$
\left\{\begin{array}{l}
\quad h_{n \psi}(z) \mid<a_{n} \cdot\left(\mathrm{I}+\frac{|z|}{a_{n}}\right) \cdot 2 c_{n} \cdot \frac{\mathrm{I}}{\left(\frac{\mathrm{I}}{\left(\frac{|z|}{a_{n}}\right)}-\mathrm{I}\right) \log a_{n}^{-1}-\mathrm{I}} \\
=\frac{2 a_{n} c_{n} \cdot\left(\mathrm{I}+\frac{|z|}{a_{n}}\right)}{\log a_{n}^{-1}} \cdot \frac{\frac{|\tilde{z}|}{a_{n}}}{\mathrm{I}-\frac{|\tilde{z}|}{a_{n}}\left(1+\frac{\mathrm{I}}{\log a_{n}^{-1}}\right)} \\
=\frac{2 a_{n} c_{n}}{\log a_{n}^{-1}}\left(\mathrm{I}+\frac{|z|}{a_{n}}\right) \cdot \sum_{r=1}^{x}\left(\frac{|z|}{a_{n}^{\prime}}\right)^{r} \cdot\left(\mathrm{I}+\frac{\mathrm{I}}{\log a_{n}^{-1}}\right)^{r-1} \\
=\frac{2 a_{n} c_{n}}{\log a_{n}^{-1}} \cdot\left[\frac{|z|}{a_{n}}+\left(2+\frac{\mathrm{I}}{\log a_{n}^{-1}}\right) \cdot \sum_{r=2}^{*}\left(\frac{|\tilde{z}|}{a_{n}}\right)^{r} \cdot\left(\mathrm{I}+\frac{\mathrm{I}}{\log a_{n}^{-1}}\right)^{r-2}\right] .
\end{array}\right.
$$

Der letzte Ausdruck in ( 50 ) ergibt nach Porenzen von $|z|$ geordnet den letzten Ausdruck in $(5 \mathrm{I})$. Setzt man $z=\bar{i}=|k|$, so sind diese Ungleichheitsheziehungen auch richrig für den Koeffizienten jeder einzelnen Potenz von $|z|$ in der Entwicklung der linken und rechten Seite. Unter Berücksichtung von $(+8)$ und (49) wird der Koeffizient von $i$ in $g_{n}(i)$ ermittelt und das entsprechende Glied von $g_{n}(z)$ abgezogen:

$$
\left|g_{n}(z)+\frac{2 i c_{n} z}{\log a_{n}^{-1}}\right|<\frac{8 c_{n}}{\log a_{n}^{-1}}\left(2+\frac{\mathrm{I}}{\log a_{n}^{-i}}\right) \cdot \frac{|z|}{a_{n}} \cdot|i| \cdot \sum_{r=0}^{\infty}\left(\frac{|z|}{a_{n}}\right)^{r} \cdot\left(\mathrm{I}+\frac{\mathrm{I}}{\log a_{n}^{-i}}\right)^{\prime} .
$$

Hierin darf man, wie ausgeführt, rechts die geraden Potenzen von $i$ fortlassen; also ist nach (27) und (45)

$$
\begin{gathered}
\left|\frac{g_{n}(\tilde{z})}{\tilde{z}}+\frac{2 i c_{n}}{\log a_{n}^{-1}}\right|<\frac{8 c_{n}}{\log a_{n}^{-1}} \cdot\left(2+\frac{1}{\log a_{n}^{-1}}\right) \cdot\left(\frac{|z|}{a_{n}}\right)^{2} \cdot \sum_{s=0}^{\infty}\left(\frac{|z|}{a_{n}}\right)^{2 s}\left(\mathrm{I}+\frac{\mathrm{I}}{\log a_{n}^{-1}}\right)^{2 s+1} \\
\quad=\frac{8 c_{n}}{\log a_{n}^{-1}} \cdot\left(2+\frac{1}{\log a_{n}^{-1}}\right) \cdot\left(\frac{|z|}{a_{n}}\right)^{2} \cdot \frac{\mathrm{I}+\frac{\mathrm{I}}{\log a_{n}^{-1}}}{\mathrm{I}-\left(\frac{|z|}{a_{n}}\right)^{2}\left(\mathrm{I}+\frac{\mathrm{I}}{\log a_{n}^{-1}}\right)^{2}} \\
\quad<\frac{8 c_{n}}{\log a_{n}^{-1}} \cdot \frac{5}{2} \cdot \frac{\frac{3}{2}}{\mathrm{I}-\left(\frac{\mathrm{I}}{2} \cdot \frac{3}{2}\right)^{2}} \cdot\left(\frac{|z|}{a_{n}}\right)^{2}=\frac{2 c_{n}}{\log a_{n}^{-1}} \cdot\left(\frac{|z|}{a_{n}}\right)^{2} \cdot \frac{240}{7}
\end{gathered}
$$

Es ist nach (36), (52), (39), (32)

$$
\left\{\begin{aligned}
S_{1} & <\sum_{n=1}^{N} \frac{2 c_{n}}{\log a_{n}^{-1}} \cdot\left(\frac{|\tilde{I}|}{a_{n}}\right)^{2} \cdot \frac{2+0}{T}<\sum_{n=1}^{N} \frac{2 c_{n}}{\log a_{n}^{-1}} \cdot\left(\frac{\delta}{a_{N}}\right)^{2} \cdot \frac{240}{7} \\
& <\sum_{n=1}^{\infty} \frac{2 i_{n}}{\log a_{n}^{-1}} \cdot \frac{7 \xi}{720 A} \cdot \frac{2 \nmid 0}{T}=\frac{\varepsilon}{3} .
\end{aligned}\right.
$$


Ferner ist nach $(36),(52),(41),(3 i)$

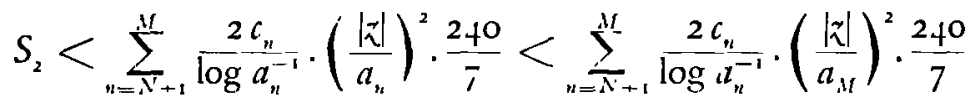

$$
\begin{aligned}
& <\sum_{n=N^{\prime}+1}^{\infty} \frac{2 c_{n}}{\log a_{n}^{-1}} \cdot\left(\frac{\mathrm{I}}{2}\right)^{2} \cdot \frac{240}{7}<\frac{7 \varepsilon}{20 \mathrm{I}} \cdot \frac{60}{7}=\frac{60 \varepsilon}{20 \mathrm{I}},
\end{aligned}
$$

ferner nach $(36)$ und $(37)$

also ist

$$
S_{4} \leq \sum_{n=\Lambda+1}^{\infty} \frac{2 i_{1}}{\log a_{n}^{-1}}<\frac{7 \varepsilon}{2 O I}
$$

$$
S_{2}+S_{t}<\frac{\varepsilon(60+7)}{20 I}=\frac{\varepsilon}{3} \text {. }
$$

Zur Abschätzung von $S_{3}$ dienen die folgenden Betrachtungen:

Es besitzt die Funktion $r \log \log r^{-1}$ den Grenzwert o für $r=0$ und nimmt den Wert o wieder an für $r=e^{-1}$. Dazwischen ist sie positiv und muss mindestens ein Maximun besitzen. Es sei

(55) o $=\frac{d}{d r}\left(r \cdot \log \log r^{-1}\right)=\log \log r^{-1}+r \cdot \frac{\mathrm{I}}{\log r^{-1}} \cdot \frac{(-\mathrm{I})}{r}=\log \log r^{-1}-\frac{\mathrm{I}}{\log r^{-1}}$.

Diese Gleichung sei für den Wert $r=r_{\text {。 }}$ erfüllt. Setzt man

so ist

$$
\log r_{0}^{-1}=l_{0}
$$

$$
l_{\mathrm{o}} \log l_{\mathrm{o}}=\mathrm{I} \text {. }
$$

Da $l_{0}>0$, so muss auch $\log l_{0}>0$, sein, also $l_{0}>$ I. Für $l_{0}>$ I wächst $l_{0} \cdot \log l_{0}$ monoton mit $l_{0}$.

Es ist

also ist

$$
\begin{gathered}
\text { 2. } \log 2=\log +>1, \\
\log r_{0}^{-1}=l_{0}<2, \\
r_{0}>e^{-2} .
\end{gathered}
$$

Da (55) im betrachteten Intervalle nur diese eine Lösung besitzt, muss bei $r_{\mathrm{o}}$ das gesuchte Maximum liegen, andere Maxinı oder Minima können im Intervalle nicht existieren; es wächst also die Funktion $r \log \log r^{-1}$ monoton im Intervall

$$
0 \leq r \leq e^{-2}
$$

Fur ein $g_{n}(z)$, das in $S$; vorkommt, ist nach (4I)

$$
|z|>\frac{a_{n}}{2} \text {. }
$$

Demnach ist

Es ist nach (10)

$$
\left|z-a_{n} \cdot i^{2}\right| \leq|z|+a_{n}<3|z| .
$$

$$
\left\{\begin{aligned}
\left|h_{n \psi}(z)\right| & <\left|z-a_{n} \cdot i^{\downarrow}\right| \cdot c_{n} \cdot\left|\log \frac{\log \left|z-a_{n} \cdot i^{\dagger}\right|^{-1} \mid}{\log a_{n}^{-1}}\right| \\
& =\left|z-a_{n} \cdot i^{-}\right| \cdot c_{n}|\log \log | z-\left.a_{n} \cdot i^{\dagger}\right|^{-1}-\log \log a_{n}^{-1} \mid
\end{aligned}\right.
$$

Man unterscheide zwci Fïlle: 
Fall (a): Es sei

$$
\left|z-a_{n} \cdot i^{*}\right| \leq a_{n}<e^{2}
$$

Dann überwiegt in der letzten Differenz in (59) das erste Glied; man benutze die Tatsache, dass $r \cdot \log \log r^{-1}$ im Intervall (56) monoton wächst, wende ferner ( 57$)$ an:

Fall (b): Es sei

$$
\begin{aligned}
\left|\frac{b_{n \psi}(z)}{z}\right| & <\frac{c_{n} \cdot\left|z-a_{n} \cdot i^{\psi}\right| \cdot \log \log \left|z-a_{n} \cdot i^{\psi}\right|^{-1}}{|z|} \\
& <\frac{c_{n} \cdot a_{n} \cdot \log \log a_{n}^{-1}}{\left(\frac{a_{n}}{2}\right)}=2 c_{n} \cdot \log \log a_{n}^{-1} .
\end{aligned}
$$

$$
\left|z-a_{n} \cdot i^{\psi}\right|>a_{n} \text {. }
$$

Dann überwiegt in der letzten Differenz in (59) das zweite Glied. Also wird nach (58)

$$
\left|\frac{b_{n \psi}(z)}{z}\right|<\frac{c_{n} \cdot\left|z-a_{n} \cdot i^{\psi}\right| \cdot \log \log a_{n}^{-1}}{|z|}<3 c_{n} \cdot \log \log a_{n}^{-1} .
$$

Es ist also allgemein

$$
\left|\frac{y_{n}(i)}{z}\right| \leq \sum_{\psi=0}^{3}\left|\frac{h_{n \downarrow}(i)}{z}\right|<12 c_{n} \cdot \log \log a_{n}^{-1} .
$$

Es folgt aber aus $\left(3^{6}\right),(60)$ und $(38)$

$$
S_{3}<\sum_{n=M+1}^{\infty} \mathrm{I} 2 c_{n} \cdot \log \log a_{n}^{-1}<12 \cdot \frac{\varepsilon}{36}=\frac{\varepsilon}{3} .
$$

Also ist nach $(53),(5+),(61)$

$$
S_{1}+S_{2}+S_{3}+S_{4}<\frac{\varepsilon}{3}+\frac{\varepsilon}{3}+\frac{\varepsilon}{3}=\varepsilon .
$$

Damit sind aber nach (36) auch (35) und (34) erfüllt.

Um die Existenz des erweiterten absoluten Differentialquotienten an der Stelle $z=0$ zu beweisen, vertahre man ähnlich.

Zunächst soll gezeigt werden, dass ein Wert $i$ nicht gleichzeitig zwei Ungleichheitsbeziehungen von der Form

$$
\begin{aligned}
& \left|z-a_{n} . i^{\psi}\right|<p_{n}, \\
& \left|z-a_{m} . i^{\psi}\right|<p_{m}
\end{aligned}
$$

genügen kann, wenn nicht $a_{n} \cdot i^{\psi}=a_{m} \cdot i^{p}$; hierin bedeuten $\psi$ und $\psi$ eine der Zahlen $0,1,2,3$. Denn aus (62) und (63) würde folgen

$$
\left\{\begin{array}{c}
\left|a_{n} \cdot i^{\psi}-a_{m} \cdot i^{\varphi}\right|=\left|\left(a_{n} \cdot i^{\psi}-z\right)-\left(a_{m} \cdot i^{\varphi}-z\right)\right| \\
\leq\left|a_{n} \cdot i^{\psi}-z\right|+\left|a_{m} \cdot i^{\varphi}-z\right|<p_{n}+p_{m} .
\end{array}\right.
$$

Wenn $n<m$, so ist nach (64), (29) und (30)

$$
\begin{aligned}
a_{n}-a_{n+1} & \leq a_{n}-a_{m} \leq\left|a_{n} \cdot i^{\psi}-a_{m} \cdot i^{\varphi}\right|<\rho_{n}+\rho_{m} \\
& \leq \rho_{n}+\rho_{n+1}<\frac{a_{n}-a_{n+1}}{2}+\frac{a_{n}-a_{n+1}}{2}=a_{n}-a_{n+1} .
\end{aligned}
$$


Diese Ungleichheitsbeziehung enthält aber einen IViderspruch. Wenn $n=m$, so ist wach $(64)$ und $(30)$

$$
a_{n} \cdot 1^{\prime} \frac{1}{2} \leq\left|a_{n} \cdot i^{\psi}-a_{n} \cdot i^{q}\right|<2 p_{n}<a_{n}-a_{n+1} .
$$

Auch diese Ungleichheitsbeziehung enthält einen Widerspruch. Also ist das gleichzeitige Bestehen von (62) und (63) für keinen Wert z möglich, d. h. je zwei Kreisgebiete (62), (63) liegen ausserhalb einander. Man unterscheide nun zwei Fälle, je nachdem es für ein Wertepaar $\tilde{i}_{1}, z_{2}$ einen bestimnten Wert $a_{m} . i^{\not}$ gibt derart, dass gleichzeitig

$$
\begin{aligned}
& \left|z_{1}-a_{m} \cdot i^{\varphi}\right|<p_{m} \\
& \left|z_{2}-a_{m} \cdot i^{\psi}\right|<p_{m}
\end{aligned}
$$

oder $(65)$ und (66) für kein $a_{m} . i^{\ddagger}$ gleichzeitig erfüllt sind. Der zweite Fall soll zuerst behandelt w'erden. Es ist

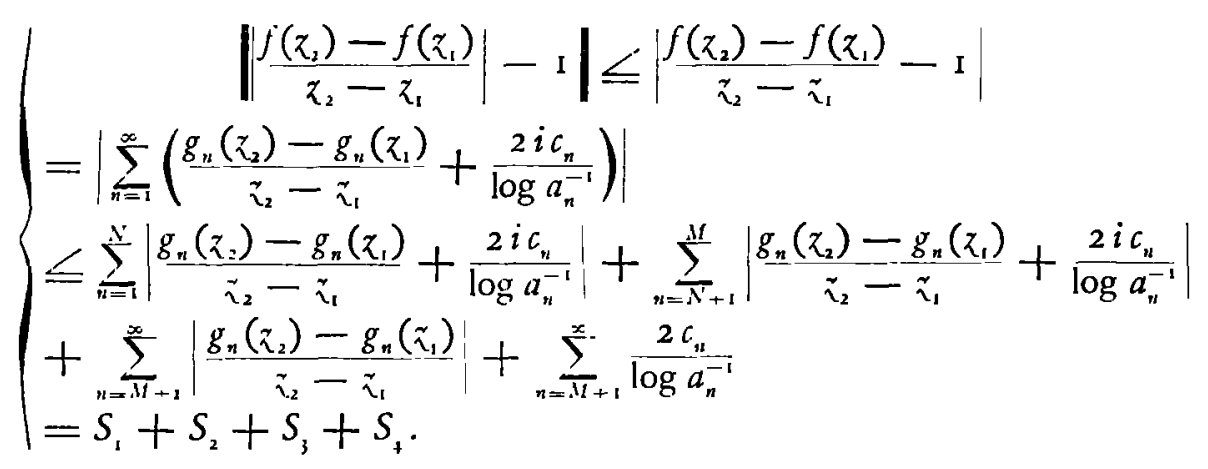

Hierin sei wieder $M \gg N$ und zwar sei $N$ so bestimmt, dass

$$
\begin{aligned}
& \sum_{n=\bar{N}+1}^{\infty} \frac{2 c_{n}}{\log a_{n}^{-1}}<\frac{49 \varepsilon}{7167}, \\
& \sum_{n=\Lambda_{+1}^{\infty}}^{\infty} c_{n} \log \log p_{n}^{-1}<\frac{\varepsilon}{20} ;
\end{aligned}
$$

sodann bestimme man

und

$$
\delta<a_{\Lambda} \cdot \sqrt{\frac{49 \varepsilon}{28080 A}}
$$

Für das vorliegende Wertepaar $\left|\tilde{k}_{1}\right| \leq\left|\tilde{z}_{2}\right|$, das absolut kleiner als $\delta$ sei, bestimme man $M$ so gross, dass

$$
\delta<\frac{a_{N^{\prime}}}{2} .
$$

Es genügt nach $\left(\gamma_{1}\right) N$ der zweiten dieser Bedingungen, es kann auch $N=M$ sein, so dass $S_{2}=0$ wird.

Zur Abschätzung von $S_{1}$ und $S_{2}$ verfahre man ganz ähnlich wie vorher; wegen 
(72) ist für $S_{1}$ und $S_{2}$

$$
\left|z_{1}\right| \leq\left|z_{2}\right| \leq \frac{a_{n}}{2}
$$

also lässt sich $(42)$ in eine konvergente Potenzreihe entwickeln. Es ist wieder ( $5 t)$ eine Majorante für $b_{n \downarrow}(z)$. Beim Uebergang zur Majorante von $g_{n}(z)$ darf man wieder in dieser die geraden Potenzen von $|\bar{\imath}|$ fortlassen. Wendet man die Abschätzung (9) an, so ergibt sich

$$
\begin{aligned}
& \left|\frac{g_{n}\left(\tilde{z}_{2}\right)-g_{n}\left(\tilde{z}_{1}\right)}{z_{2}-\tilde{z}_{1}}+\frac{2 i c_{n}}{\log a_{n}^{-1}}\right| \\
& <\frac{8 c_{n}}{\log a_{n}^{-1}} \cdot\left(2+\frac{1}{\log a_{n}^{-1}}\right) \cdot \sum_{s=1}^{\infty}(2 s+1) \cdot\left(\frac{|z|}{a_{n}}\right)^{2 s} \cdot\left(1+\frac{1}{\log a_{n}^{-1}}\right)^{2 s-1} \\
& =\frac{8 c_{n}}{\log a_{n}^{-1}}\left(2+\frac{1}{\log a_{n}^{-1}}\right) \cdot\left(\frac{|z|}{a_{n}}\right)^{2}\left[\frac{\dot{2}^{2}\left(1+\frac{1}{\log a_{n}^{-1}}\right)}{\left\{1-\left(\frac{|\tilde{z}|}{a_{n}}\right)^{2} \cdot\left(1+\frac{1}{\log a_{n}^{-1}}\right)^{2}\right\}^{2}}\right. \\
& \left.+\frac{\mathrm{I}+\frac{\mathrm{I}}{\log a_{n}^{-1}}}{\mathrm{I}-\left(\frac{|z|}{a_{n}}\right)^{2} \cdot\left(\mathrm{I}+\frac{\mathrm{I}}{\log a_{n}^{-1}}\right)^{2}}\right] \\
& <\frac{8 c_{n}}{\log a_{n}^{-1}} \cdot \frac{5}{2} \cdot\left(\frac{|\Sigma|}{a_{n}}\right)^{2} \cdot\left[\frac{3}{\left\{1-\left(\frac{1}{2} \cdot \frac{3}{2}\right)^{2}\right\}^{2}}+\frac{\frac{3}{2}}{\mathrm{I}-\left(\frac{\mathrm{I}}{2} \cdot \frac{3}{2}\right)^{2}}\right] \\
& =\frac{2 c_{n}}{\log a_{n}^{-1}}\left(\frac{|z|}{a_{n}}\right)^{2} \cdot \frac{9360}{49} \text {. }
\end{aligned}
$$

Es ist nach $(67),(73),(70),(32)$

$$
\left\{\begin{aligned}
S_{1} & <\sum_{n=1}^{N} \frac{2 c_{n}}{\log a_{n}^{-1}} \cdot\left(\frac{|z|}{a_{n}}\right)^{2} \cdot \frac{9360}{49}<\sum_{n=1}^{N} \frac{2 c_{n}}{\log c_{n}^{-1}} \cdot\left(\frac{\delta}{a_{N^{\prime}}}\right)^{2} \cdot \frac{9360}{49} \\
& \quad<\sum_{n=1}^{\infty} \frac{2 c_{n}}{\log a_{n}^{-1}} \cdot \frac{49 \varepsilon}{28080 A} \cdot \frac{9360}{49}=\frac{\varepsilon}{3} .
\end{aligned}\right.
$$

Es ist ferner nach $(67),(73),(72),(68)$

$$
\left\{\begin{array}{c}
S_{2}<\sum_{n=N+1}^{M} \frac{2 c_{n}}{\log a_{n}^{-1}} \cdot\left(\frac{|z|}{a_{n}}\right)^{2} \frac{9360}{49}<\sum_{n=N+1}^{M} \frac{2 c_{n}}{\log a_{n}^{-1}}\left(\frac{|z|}{a_{M}}\right)^{2} \cdot \frac{9360}{49} \\
\leq \sum_{n=N+1}^{\infty} \frac{2 c_{n}}{\log a_{n}^{-1}} \cdot\left(\frac{\mathrm{I}}{2}\right)^{2} \cdot \frac{9360}{49}<\frac{49 \varepsilon}{7167} \cdot \frac{2340}{49}=\frac{2340 \varepsilon}{7167}
\end{array}\right.
$$

ferner wach $\left(6_{7}\right)$ und $(68)$

also ist

$$
S_{4} \leq \sum_{n=N^{+}+1}^{\infty} \frac{2 c_{n}}{\log a_{n}^{-1}}<\frac{49 \varepsilon}{7167}
$$

$$
S_{2}+S_{4}<\frac{\varepsilon(2340+49)}{7^{167}}=\frac{\varepsilon}{3} \text {. }
$$


Es ist ferner

(ii)

Man setze

$(78)$

$$
S_{;} \leq \sum_{n=\eta_{+1}}^{\infty} \sum_{\psi=0}^{3}\left|\frac{b_{n \psi}\left(\tilde{i}_{2}\right)-b_{n \psi}\left(\tilde{i}_{1}\right)}{z_{2}-z_{1}}\right|=\bar{S}_{3} .
$$

$$
\begin{aligned}
& z_{1}-a_{n} \cdot i_{+}=1 \zeta_{\alpha}^{\prime}, \\
& z_{2}-a_{n} \cdot i_{+}=1 \zeta_{\dot{p}},
\end{aligned}
$$

und zwar verfüge man über die Indices $x$ und $\xi$ so, dass

$$
\left.\left|\zeta_{\beta}\right| \gg\right|_{\sigma_{\alpha}} ^{\varphi} \mid \text {. }
$$

$\mathrm{Da}(65)$ und (66) nicht gleichzeitig erfüllt sollen, so ist

(79)

Es wird

$$
\left|\zeta_{\beta}\right| \searrow_{i_{n}} \text {. }
$$

(8o)

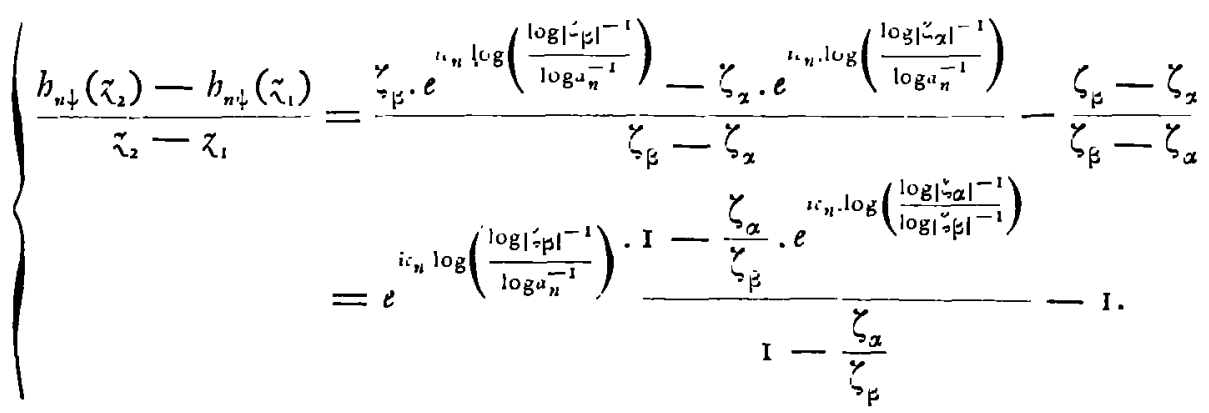

Es sei $\alpha$ eine reelle Zahl, $a$ eine komplexe Zahl, dann ist nach (ro)

$$
\left\{\begin{array}{l}
\left|e^{i \alpha} \cdot a-\mathrm{I}\right|=\left|\left(e^{i \alpha} \cdot a-e^{i \alpha}\right)+\left(e^{i x}-\mathrm{I}\right)\right| \\
\leq\left|e^{i \alpha}\right| \cdot|a-\mathrm{I}|+\left|e^{i \alpha}-\mathrm{I}\right|<|a-\mathrm{I}|+|x| .
\end{array}\right.
$$

Wendet man ( $8 \mathrm{I}$ ) auf den letzten Ausdruck in (8o) an, so erhält man

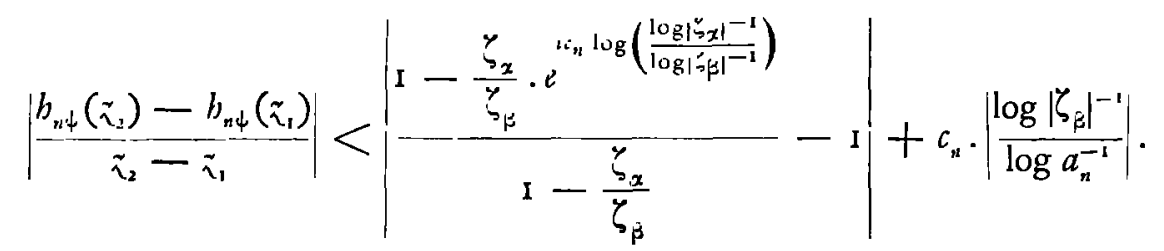

Zur Abschätzung des ersten Ausdrucks auf der rechten Seite benutze man (I6) vom dritten Ausdruck an und (19). Der zweite Ausdruck ist gleich

$\mathrm{Da}$ nach (79)

da ferner nach (30)

$$
\left.c_{n} \cdot|\log \log | \gamma_{\beta}\right|^{-1}-\log \log \left|a_{n}\right|^{-1} \mid
$$

$$
\log \log \left|\zeta_{\beta}\right|^{-1} \leq \log \log p_{n}^{-1}
$$$$
\log \log a_{n}^{-1}<\log \log p_{n}^{-1}
$$

und eine Differenz zweier positiver Grössen absolut nicht grösser sein kann als die grössere der beiden, so erhält man aus (82)

$$
\left|\frac{h_{n \psi}\left(z_{2}\right)-h_{n \psi}\left(z_{1}\right)}{z_{2}-z_{1}}\right|<\frac{c_{n}}{\log \left|\zeta_{\xi}\right|^{-1}}+c_{n} \cdot \log \log \rho_{n}^{-1} .
$$


Hierin ist nach $(78),(72),(27)$

$$
\left|\zeta_{\beta}\right| \leq|z|+a_{n} \leq \frac{3}{2} a_{n} \leq \frac{3}{2 e^{2}}
$$

ferner ist nach (30)

$$
p_{n}<\frac{a_{n}}{2}<\frac{1}{2 e^{2}}
$$

Also erhält man aus $\left(8_{3}\right)$ unter Benutzung von $(84)$ und $(8 ;)$

$$
\left\{\begin{array}{c}
\left|\frac{b_{n \psi}\left(\tilde{i}_{2}\right)-b_{n \psi}\left(\tilde{i}_{1}\right)}{z_{2}-i_{1}}\right| \\
<\frac{i_{n}}{\log \left(\frac{2 e^{2}}{3}\right)} \cdot \frac{\log \log p_{n}^{-1}}{\log \log \left(2 e^{2}\right)}+c_{n} \cdot \log \log p_{n}^{-1}<\frac{5}{3} c_{n} \cdot \log \log p_{n}^{-1} .
\end{array}\right.
$$

Also folgt aus $(77),(86)$ und (69)

$$
S_{i} \leq \bar{S}_{3}<\sum_{n=1 \pi+1}^{\infty} \frac{20}{3} \cdot c_{n} \log \log p_{n}^{-1}<\frac{20}{3} \cdot \frac{\varepsilon}{20}=\frac{\varepsilon}{3} .
$$

Also ergibt sich aus $(67),(74),(76),(87)$

$$
|| \frac{f\left(\tilde{i}_{2}\right)-f\left(\tilde{i}_{1}\right)}{\tilde{i}_{2}-\tilde{i}_{1}}|-\mathrm{I}| \leq S_{1}+S_{2}+\bar{S}_{3}+S_{+}<\frac{\varepsilon}{3}+\frac{\varepsilon}{3}+\frac{\varepsilon}{3}=\varepsilon .
$$

In dem noch zu behandelnden ersten Falle waren für ein bestimmtes $a_{m} . i^{7}(65)$ und (66) gleichzeitig erfullt; m.un setze

so dass

$$
\begin{aligned}
& z_{1}-a_{m} \cdot i^{\phi}=\mid Z_{x} \\
& \tilde{i}_{2}-a_{m} \cdot i^{\psi}=\left\{Z_{\beta}\right. \text {, }
\end{aligned}
$$

(89)

$$
\left|Z_{\alpha}\right| \leq\left|Z_{j}\right|<\rho_{m}
$$

Zunächst ist nach $\left(7^{2}\right),(66),(30)$

also ist

$$
\frac{a_{M}}{2} \geqslant\left|i_{2}\right|>a_{m}-p_{m}>a_{m}-\frac{a_{m}}{2}=\frac{a_{m}}{2},
$$

(90)

Man setze zur Abkürzung

$$
a_{m} \leq a_{M+1}
$$

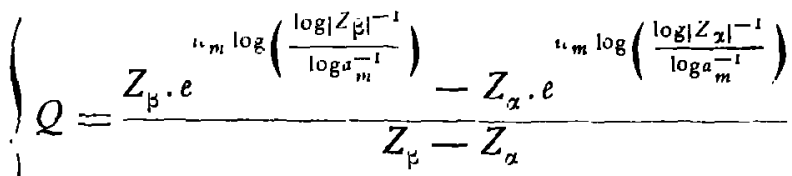

$$
\begin{aligned}
& \Lambda=\frac{b_{m \varphi}\left(\tilde{z}_{2}\right)-b_{m \uparrow}\left(z_{1}\right)}{z_{2}-i_{1}}+1 .
\end{aligned}
$$

Da für alle $a_{n} \cdot i^{\psi}$, die von $a_{m} \cdot i^{\dagger}$ verschieden sind, weder für $z_{1}$ noch für $z_{2}(62)$ erfüllt sein kann, und nach (90) $a_{m}$ erst in $S_{i}$ vorkommt, so gelten $(7+)$ und $(75)$ unverändert. In $S_{\text {; }}$ und $\overline{S_{i}}$ lasse man das Glied mit $b_{m{ }^{\prime} \phi}$ zunächst weg; für die übrigen Glieder gilt (79) bis (86). Unter Anwendung voll (74), (76), von (86) mit der angegebenen Weglassung, ferner von (16) und (19) zur Abschätzung von ||$Q_{1}-1 \|$ und sihliesslich 
unter Awwendung von (87), (88) und (89) erhält man

(92)

$$
\begin{aligned}
& || \frac{f\left(z_{2}\right)-f\left(z_{1}\right)}{z_{2}-z_{1}}|-1|=\left|\left(\left|\frac{f\left(z_{2}\right)-f\left(z_{1}\right)}{z_{2}-z_{1}}\right|-|Q|\right)+(|Q|-1)\right| \\
& \leq\left|\frac{f\left(z_{2}\right)-f\left(z_{1}\right)}{z_{2}-z_{1}}-Q\right|+|| Q|-1| \\
& \left\{<S_{i}+S_{2}+\bar{S}_{j}-\left|\frac{h_{m q}\left(z_{2}\right)-b_{m p}\left(z_{1}\right)}{z_{1}-i_{1}}\right|+S_{4}+\frac{c_{m}}{\log \left|Z_{\beta}\right|-1}\right. \\
& <S_{1}+S_{2}+4 \sum_{n=M+1}^{\infty}\left(\frac{c_{n}}{\log \left|\zeta_{\beta}\right|^{-1}}+c_{n} \cdot \log \log P_{n}^{-1}\right) \\
& -\left(\frac{c_{m}}{\log \left|Z_{\bar{\beta}}\right|^{-1}}+c_{m} \cdot \log \log \rho_{m}^{-1}\right)+S_{+}+\frac{c_{m 1}}{\log \left|Z_{\beta}\right|^{-1}}<\varepsilon .
\end{aligned}
$$

Damit ist die Existenz des erweiterten absoluten Differentialquotienten an der Stelle $z=0$ nachgewiesen.

Es soll gezeigt werden, dass kein verallgemeinerter Signum-Differentialquorient vorhanden ist, dass man also nach Annalınıe einer beliebig kleinen positiven Grösse $\bar{\delta}$, die kleiner oder gleich dem zu $\varepsilon=\frac{24}{25}$ gehörigen $\delta$ sei, $\left|z_{1}\right|<\bar{\delta}$ und $\left|z_{2}\right|<\bar{\delta}$ so bestimmen kann, dass, wenn man

$$
\frac{f\left(z_{2}\right)-f\left(z_{1}\right)}{z_{2}-z_{1}}=-1-a
$$

setzt, sgn (- I - $-a)$ sich vou I um mehr als eine feste endliche Grösse unterscheidet. Man bestimme

$$
a_{m}<\frac{2}{3} \bar{\delta}
$$

Es seien (65) und (66) gleichzeitig erfüllt; dann ist

$$
z_{2} \leq a_{m}+p_{m}<\frac{3}{2} a_{m}<\bar{\delta} .
$$

Man setze $z_{\mathrm{r}}=a_{m}$, also $Z_{\alpha}=0$; dann wird

$$
Q=e^{r_{m} \log \left(\frac{\left.\log Z_{\beta}\right|^{-1}}{\log _{m+1}^{-1}}\right)} \text {. }
$$

Man kann nun über $Z_{\beta}$ so verfügen, dass $Q=-1$ wird. Also ist nach (92)

$$
|a|=|(-1-a)-Q|+|| Q|-1|<\frac{24}{25} \text {. }
$$

Nun ist aber

(94)

$$
|\operatorname{sgn}(1+a)-1|^{2}+|\operatorname{sgn}(1+a)+1|^{2}=4,
$$

also ist nach (94), (23), (93)

$$
\begin{gathered}
|\operatorname{sgn}(-1-a)-1|=|\operatorname{sgn}(1+a)+1|=1^{\prime}-|\operatorname{sgn}(1+a)-1|^{2} \\
\quad \geq \mid \frac{1}{2}+2 \sqrt{1-|a|^{2}}>\sqrt{2+2 \sqrt{1-\left(\frac{24}{25}\right)^{2}}}=\frac{8}{5} .
\end{gathered}
$$

Also existiert kein erw'eiterter Signum-Differentialquotient. 
Beispiel $(H)$ : Es seien zwei monoton gegen o abnehmende Reihen reeller positiver Zahlen gegeben,

die der Bedingung genügen

$$
\lim _{n=\infty} a_{n}=0, \quad \lim _{n=\infty} b_{n}=0,
$$

$$
a_{n}<\frac{\mathrm{I}}{2 e}
$$

es konvergiere ferner die Reihe

also konvergiert auch

$$
\sum_{n=1}^{x} 4 b_{n} \log a_{n}^{-1}=A
$$

$$
\sum_{n=1}^{\infty} 2 b_{n}=B
$$

Es sei $f(z)$ im Gebiete

$$
|\tilde{k}|<\frac{\mathrm{I}}{2 e}
$$

definiert durch die Gleichung

Hierin ist

$$
f(z)=B \cdot z+\sum_{n=1}^{\infty} g_{n}(z)
$$

hierin wieder

$$
g_{n}(z)=b_{n} \cdot \sum_{\psi=0}^{3} b_{n \psi}(\tilde{i}),
$$

$$
\begin{aligned}
b_{n \psi}(z) & =\left(z-a_{n} \cdot i^{\psi}\right) \cdot \log \left|z-a_{n} \cdot i^{\psi}\right|^{-1} \\
& =a_{n} \cdot i^{\psi} \cdot\left(\frac{z}{a_{n} \cdot i^{\psi}}-\mathrm{I}\right) \cdot\left[\log \left|\mathrm{I}-\frac{z}{a_{n} \cdot i^{\psi}}\right|^{-1}+\log a_{n}^{-1}\right] .
\end{aligned}
$$

Es ist

$$
b_{n \downarrow}(0)=-a_{n} \cdot i^{\downarrow} \cdot \log a_{n}^{-1}, \quad g_{n}(0)=-b_{n} a_{n} \log a_{n}^{-1} \cdot \sum_{\psi=0}^{3} i^{\psi}=0,
$$

also

$$
f(0)=0 .
$$

Es soll zunächst gezeigt werden, dass $f(z)$ an der Stelle $i=0$ den gewöhnlichen Differentialquotienten $A$ besitzt. Es soll also gezeigt werden, dass

$$
\lim _{i=0}\left(\frac{f(z)}{z}-A\right)=0
$$

D. h. nach Annahıne einer beliebig kleinen reellen positiven Grösse $\varepsilon$ soll eine reelle positive Grösse $\delta$ hinreichend klein so bestimmt werden, dass für $|z|<\delta$

(99) $\left|\frac{f(z)}{z}-A\right|=\left|\sum_{n=1}^{\infty} \frac{g_{n}(z)}{z}+B-A\right|=\left|\sum_{n=1}^{\infty}\left(\frac{g_{n}(z)}{z}+2 b_{n}-4 b_{n} \log a_{n}^{-1}\right)\right|<\varepsilon$ wird. Es mögen $M \supseteq N$ zwei noch zu bestimmende positive ganze Zahlen sein. Dann ist

$$
(100)\left\{\begin{array}{l}
\left|\sum_{n=1}^{\infty}\left(\frac{g_{n}(z)}{z}+2 b_{n}-4 b_{n} \cdot \log a_{n}^{-1}\right)\right| \\
\leq \sum_{n=1}^{N}\left|\frac{g_{n}(z)}{z}+2 b_{n}-4 b_{n} \cdot \log a_{n}^{-1}\right|+\sum_{n=N+1}^{M}\left|\frac{g_{n}(z)}{z}+2 b_{n}-4 b_{n} \log a_{n}^{-1}\right| \\
+\sum_{n=\bar{N}+1}^{\infty}\left|\frac{g_{n}(z)}{z}\right|+\sum_{n=M+1}^{\infty} 2 b_{n}+\sum_{n=M+1}^{\infty}+b_{n} \log a_{n}^{-1} \\
=S_{1}+S_{2}+S_{j}+S_{+}+S_{j} .
\end{array}\right.
$$


Hierin sei $N$ so bestimmt, dass

(IOI)

$$
\sum_{n=N_{+1}}^{5}+b_{n} \cdot \log a_{n}^{-1}<\frac{3 \varepsilon}{26}
$$

woraus unter Benutzung von (95) folgt

$$
\sum_{n=\Lambda+1}^{\infty} 2 b_{n}<\sum_{n=\Lambda+1}^{\infty} \frac{+b_{n} \cdot \log a_{n}^{-1}}{2 \cdot \log 2 e}<\frac{3}{10} \cdot \frac{3 \cdot \varepsilon}{26}=\frac{9 \varepsilon}{260} .
$$

Sodann bestimne man

$$
\delta<a_{x} \cdot \sqrt{\overline{9 \Xi}}
$$

und

(104)

$$
\delta<\frac{a_{\Lambda^{\prime}}}{2} .
$$

Für das vorliegende $i$, das absolut kleiner als $\delta$ sei, bestimme man $M$ so gross, dass

$$
\frac{a_{M+1}}{2}<|\tilde{k}| \leq \frac{a_{M}}{2}
$$

Es genügt nach (Iof) $N$ der zweiten dieser Bedingungen, es kinn auch $M=N$ sein, so dass $S_{2}=0$ wird.

Zur Abschätzung von $S_{1}$ und $S_{2}$ verfabre man folgendermassen. Es ist

$$
\begin{aligned}
& g_{n}(z)-4 b_{n} z \cdot \log a_{n}^{-1}=b_{n} \cdot \sum_{i=0}^{3}\left(z-a_{n} \cdot i^{\psi}\right) \sum_{v=1}^{x} \frac{1}{2 v}\left[\left(\frac{z}{a_{n} \cdot i^{\psi}}\right)^{v}+\left(\frac{\bar{z}}{a_{n} i^{-\psi}}\right)^{v}\right]^{\prime} \\
& =b_{n} i-2 z+\sum_{\psi=0}^{3}\left[z \cdot \sum_{v=1}^{\infty}\left(\frac{i}{a_{n}}\right)^{v} \cdot\left(\frac{i^{-\psi v}}{2 v}-\frac{i^{-\psi v}}{2(v+\mathrm{I})}\right)\right. \\
& \left.+z \cdot \sum_{v=1}^{\infty}\left(\frac{\bar{z}}{a_{n}}\right)^{v} \cdot \frac{i^{i v}}{2 v}-\bar{z} \cdot \sum_{v=0}^{\infty}\left(\frac{\vec{z}}{a_{n}}\right)^{\nu} \cdot \frac{i^{i+v+21}}{2(v+1)}\right]^{\prime} \\
& =b_{n} !-2 i+2 \sum_{\mu=1}^{\infty}\left(i \cdot\left(\frac{i}{a_{n}}\right)^{+\mu} \cdot \frac{1}{4 \mu(+\mu+I)}+i \cdot\left(\frac{\bar{z}}{a_{n}}\right)^{+\mu} \frac{I}{4 \mu}-\bar{i}\left(\frac{\bar{i}}{a_{n}}\right)^{+\mu-2} \cdot \frac{1}{4 \mu-1}\right)^{\prime}
\end{aligned}
$$

Also ergibt sich bei Benutzung von ( $\left.1 \mathrm{O}_{+}\right)$

$$
(106)\left\{\begin{array}{c}
\left|\frac{g_{n}(\tilde{i})}{i}+2 b_{n}-+b_{n} \cdot \log a_{n}^{-1}\right|<\frac{2}{3} \cdot\left(\frac{|z|}{a_{n}}\right)^{2} \cdot b_{n} \cdot \sum_{\mu=0}^{x}\left(\frac{|z|}{a_{n}}\right)^{i \mu} \\
=\frac{2}{3} \cdot b_{n} \cdot\left(\frac{|z|}{a_{n}}\right)^{2} \cdot \frac{\mathrm{I}}{1-\left(\frac{|\tilde{z}|}{a_{n}}\right)^{2}}<\frac{2}{3} \cdot b_{n} \cdot\left(\frac{|z|}{a_{n}}\right)^{2} \cdot \frac{\mathrm{I}}{\mathrm{I}-\left(\frac{1}{2}\right)^{2}}=\frac{8}{9} \cdot b_{n} \cdot\left(\frac{|z|}{a_{n}}\right)^{2} .
\end{array}\right.
$$

Es ist also nach (100), (106), (103), (96)

$(107)$

$$
\left\{\begin{aligned}
S_{1} & <\sum_{n=1}^{1} 2 b_{n} \cdot\left(\frac{|z|}{a_{n}}\right)^{2} \cdot \frac{4}{9}<\sum_{n=1}^{1} 2 b_{n} \cdot\left(\frac{\delta}{a_{N}}\right)^{2} \cdot \frac{4}{9} \\
& <\sum_{n=1}^{\infty} 2 b_{n} \cdot \frac{9 \varepsilon}{8 B} \cdot \frac{4}{9}=\frac{\varepsilon}{2} .
\end{aligned}\right.
$$


Ferner ist nach (100), (106), (105), (102)

Nach (IOO) und (IO2) ist

$$
\begin{aligned}
S_{2}<\sum_{n=N^{\prime}+1}^{M} 2 b_{n} \cdot\left(\frac{\mid \tilde{}}{a_{n}}\right)^{2} \cdot \frac{4}{9} & <\sum_{n=\Lambda+1}^{M} 2 b_{n}\left(\frac{|z|}{a_{M}}\right)^{2} \cdot \frac{4}{9} \\
& <\sum_{n=N^{\prime}+1}^{\infty} \frac{2 b_{n}}{9}<\frac{\varepsilon}{260} .
\end{aligned}
$$

also ist

(108)

$$
S_{4}<\frac{9 \varepsilon}{260}
$$

Zur Abschätzung von $S_{\text {; }}$ dienen folgende Betrachtungen. Es besitzt die Funktion $r \log r^{-1}$ fur $r=\mathrm{o}$ den Grenzwert $o$ und nimmt den Wert o wieder an für $r=\mathrm{I}$. Dazwischen muss sie mindestens ein Maximum besitzen. Es sei

$$
\mathrm{o}=\frac{d}{d r}\left(r \log r^{-1}\right)=\log r^{-1}-\mathrm{I} .
$$

Diese Gleichung ist erfüllt für $r=e^{-1}$. Da sie nur eine Lösung besitzt, nuss bei $r=e^{-1}$ das gesuchte Maximum liegen; imnerhalb des Intervalls o $<r<\mathrm{I}$ können weitere Maxima oder Minima nicht existieren, also wächst die Funktion $r \cdot \log r^{-1}$ in Intervall

$$
0 \leq r \leq e^{-1}
$$

monoton, es ist also für $S_{3}$ nit Rücksicht auf (95), (97) und (105)

$$
\begin{aligned}
\left|g_{n}(z)\right| & \leq b_{n} \cdot \sum_{\psi=0}^{3}\left|z-a_{n} \cdot i^{\psi}\right| \cdot \log \left|z-a_{n} \cdot i^{\psi}\right|^{-1} \\
& \leq{ }^{+} b_{n} \cdot\left(|z|+a_{n}\right) \cdot \log \left(|z|+a_{n}\right)^{-1}<\mathrm{I} 2 b_{n}|z| \cdot \log a_{n}^{-1} .
\end{aligned}
$$

Also ist nach (IOI)

Nach (IOI) ist

$$
S_{3}<\sum_{n=M+1}^{\infty} 12 b_{n} \cdot \log a_{n}^{-1}<\frac{9 \varepsilon}{26} .
$$

$$
S_{s}<\frac{3 \varepsilon}{26}
$$

Also ist nach dem Anfang von (99), (100), (107), (108), (I09), (I I0)

$$
\left|\frac{f(\tilde{z})}{z}-A\right|<\frac{\varepsilon}{2}+\frac{\varepsilon}{26}+\frac{9 \varepsilon}{26}+\frac{3 \varepsilon}{26}=\frac{\varepsilon}{2}+\frac{\varepsilon}{2}=\varepsilon .
$$

Damit ist (98) bewiesen.

Um die Existenz des erweiterten Signum-Differentialquotienten an der Stelle $z=0$ nachzuweisen, benutze ich folgenden Hilfssatz.

Hilfssatz I. - Es sei

$$
t_{1}, t_{2}, t_{3}, \ldots
$$

eine unendliche Reibe komplexer Zablen, die der Bedingung genïgen

$$
\left|\operatorname{sgn} t_{\mathrm{v}}-\mathrm{I}\right|<\varepsilon<\mathrm{I} \text {; }
$$


es konvergiere ferner

(11 12 )

$$
\sum_{v=1}^{\infty} t_{v}=T
$$

Dann ist auch

$$
\text { (I 13) } \quad|\operatorname{sgn} T-\mathrm{I}|<\varepsilon \text {. }
$$

Es folgt aus (III)

also ist

$$
\left|\operatorname{sgn} t_{v}-\mathrm{I}\right|^{2}=\left(\operatorname{sgn} t_{v}-\mathrm{I}\right)\left(\operatorname{sgn} \bar{t}_{v}-\mathrm{I}\right)=2-2 \Re\left(\operatorname{sgn} t_{v}\right)<\varepsilon^{2},
$$

(I I 4)

$$
\Re\left(\operatorname{sgn} t_{v}\right)>I-\frac{\varepsilon^{2}}{2}>\frac{I}{2}
$$

oder

$$
\begin{gathered}
\frac{\Re^{2}\left(\operatorname{sgn} t_{v}\right)}{\Re^{2}\left(\operatorname{sgn} t_{v}\right)+\mathfrak{S}^{2}\left(\operatorname{sgn} t_{v}\right)}>\left(\mathrm{I}-\frac{\varepsilon^{2}}{2}\right)^{2}, \\
\frac{\left|\mathfrak{J}\left(\operatorname{sgn} t_{v}\right)\right|}{\mathfrak{R}\left(\operatorname{sgn} t_{v}\right)}<1 / \frac{\mathrm{I}}{\left(\mathrm{I}-\frac{\varepsilon^{2}}{2}\right)^{2}}-\mathrm{I},
\end{gathered}
$$

(I I S)

$$
\left|\Im\left(t_{v}\right)\right|<\Re\left(t_{v}\right) \cdot \frac{\varepsilon \sqrt{4-\varepsilon^{2}}}{2-\varepsilon^{2}}
$$

Aus (II2) und (IIS) folgt

$$
\begin{gathered}
|\Im(T)| \leq \sum_{v=1}^{\infty}\left|\Im\left(t_{v}\right)\right|<\Re(T) \cdot \frac{\varepsilon l^{\prime}-\varepsilon^{2}}{2-\varepsilon^{2}}, \\
\frac{\Re^{2}(T)+\Re^{2}(T)}{\Re^{2}(T)}<\frac{\varepsilon^{2}\left(4-\varepsilon^{2}\right)}{\left(2-\varepsilon^{2}\right)^{2}}+1=\frac{4}{\left(2-\varepsilon^{2}\right)^{2}}, \\
\Re(\operatorname{sgn} T)>I-\frac{\varepsilon^{2}}{2},
\end{gathered}
$$$$
|\operatorname{sgn} T-\mathrm{I}|=1 / \overline{2-2 \Re(\operatorname{sgn} T)}<\varepsilon .
$$

Damit ist (I I 3) bewiesen.

Es ist

(II6) $\left\{\begin{array}{c}\frac{f\left(z_{2}\right)-f\left(z_{1}\right)}{z_{2}-z_{1}} \\ =\sum_{n=1}^{N}\left(\frac{\left.g_{n}\left(z_{2}\right)-\frac{g_{n}\left(z_{1}\right)}{z_{2}-z_{1}}+2 b_{n}\right)+\sum_{n=N^{\prime}+1}^{\infty} \sum_{\psi=0}^{3} \frac{b_{n \psi}\left(z_{2}\right)-b_{n \psi}\left(z_{1}\right)}{z_{2}-z_{1}}+\sum_{n=N^{\prime}+1}^{\infty} 2 b_{n}}{=S_{1}+S_{2}+S_{3} .}\right.\end{array}\right.$

Hierin sei $N$ so bestimmt, dass

( 117 )

$$
\log \left(\frac{3}{2} a_{\lambda+1}\right)^{-1}>\frac{1}{\varepsilon \sqrt{1-\frac{\varepsilon^{2}}{4}}},
$$

sodann sei

$$
\delta<a_{N} \cdot \sqrt{\frac{45 \varepsilon \sqrt{1-\frac{\varepsilon^{2}}{4}}}{22}}
$$


und

(II9)

$$
\delta<\frac{a_{N}}{2}
$$

bestimmt. Es seien

$$
\left|z_{1}\right|<\delta \text { und }\left|z_{2}\right|<\delta \text {. }
$$

Setzt man

$$
a=\frac{1}{4 b_{n} \cdot \log a_{n}^{-1}} \cdot\left(\frac{g_{n}\left(z_{2}\right)-g_{n}\left(z_{1}\right)}{z_{2}-z_{1}}+2 b_{n}\right)-1,
$$

beachtet man, dass das zwcite Glied in ( 106 ) eine Majorante des ersten Gliedes ist und wendet man die Abschätzung (9) an, so erhält man unter Benutzung von (1 I8) für jedes Glied von $S_{\text {, }}$

$$
\begin{aligned}
|a| & <\frac{1}{4 b_{n} \cdot \log a_{n}^{-1}} \cdot \frac{2}{3} b_{n} \cdot \sum_{\mu=1}^{\infty}(2 \mu+1)\left(\frac{|z|}{a_{n}}\right)^{2 \mu} \\
& =\frac{1}{6 \log a_{n}^{-1}} \cdot\left(\frac{|z|}{a_{n}}\right)^{2} \cdot\left\{\frac{2}{\left[\mathrm{I}-\left(\frac{|z|}{a_{n}}\right)^{2}\right]^{2}}+\frac{\mathrm{I}}{\left.\mathrm{I}-\left(\frac{|z|}{a_{n}}\right)^{2}\right\}}\right. \\
& <\frac{\mathrm{I}}{6 \log a_{n}^{-1}} \cdot\left(\frac{|z|}{a_{n}}\right)^{2} \cdot \mid\left[\frac{\left.\mathrm{I}-\left(\frac{\mathrm{I}}{2}\right)^{2}\right]^{2}}{\left[\mathrm{I}-\left(\frac{\mathrm{I}}{2}\right)^{2}\right.} \mid\right. \\
& =\frac{22}{27} \cdot \frac{1}{\log a_{n}^{-1}} \cdot\left(\frac{|z|}{a_{n}}\right)^{2}<\frac{22}{27} \cdot \frac{3}{5} \cdot \frac{45 \varepsilon \sqrt{1-\frac{\varepsilon^{2}}{4}}}{22}=\varepsilon \sqrt{1-\frac{\varepsilon^{2}}{4}} .
\end{aligned}
$$

Also ist nach (26)

( 120$)$

$$
\left|\operatorname{sgn}\left(\frac{g_{n}\left(z_{2}\right)-g_{n}\left(z_{1}\right)}{z_{2}-z_{1}}+2 b_{n}\right)-\mathrm{I}\right|=|\operatorname{sgn}(\mathrm{I}+a)-\mathrm{I}|<\varepsilon .
$$

Für jedes Glied von $S_{2}$ ist nach (119)

$$
\left|z-a_{n} \cdot i^{\psi}\right| \leq|z|+a_{n}<\delta+a_{N}<\frac{3}{2} a_{N},
$$

also ist nach ( 1 I 7 )

$$
\log \left|z-a_{n} \cdot i^{\psi}\right|^{-1} \geqslant \log \left(\frac{3}{2} a_{N+1}\right)^{-1}>\frac{1}{\varepsilon \sqrt{1-\frac{\varepsilon^{2}}{4}}}
$$

somit ist nach (24), (25), (26)

$$
\left|\operatorname{sgn} \frac{h_{n \psi}\left(z_{2}\right)-b_{n \psi}\left(z_{1}\right)}{z_{2}-z_{1}}-1\right|<\varepsilon .
$$

Für jedes Glied von $S_{3}$ ist ( 124 )

$$
\left|\operatorname{sgn} 2 b_{n}-\mathrm{I}\right|=0 \text {. }
$$

Also folgt aus (I I6), ( I 20), (I 23), (I 24) bei Anwendung von (I I 3)

$$
\left|\operatorname{sgn} \frac{f\left(z_{2}\right)-f\left(z_{1}\right)}{z_{2}-z_{1}}-1\right|<\varepsilon .
$$

Damit ist die Existenz des erweiterten Signum-Differentialquotienten an der Stelle $z=0$ nachgewiesen. 
Um schliesslich zu zeigen, dass $f(i)$ an der Stelle $i=0$ keinen erweiterten absoluten Differentialquotienten besitzt, zeige man, dass nach Anuahme einer beliebig kleinen Grösse $\bar{\delta}$

$$
\left|\tilde{z}_{1}\right|<\bar{\delta} \text { und }\left|\tilde{z}_{2}\right|<\bar{\delta}
$$

so bestimmt werden können, dass

$$
\left|\frac{f\left(i_{2}\right)-f\left(z_{1}\right)}{\tilde{i}_{2}-z_{1}}\right|>G
$$

wo $G$ eine beliebig grosse vorgegebene reelle positive Zahl.

Für irgend ein festes $\varepsilon<$ I werde nach (II 7 ), (II 8 ), (II9) $N$ und $\delta$ bestimnt und $\bar{\delta} \leq \bar{\delta}$ angenommen. Man wähle

worin

$$
\tilde{i}_{1}=a_{m} \cdot i^{\varphi}
$$

$$
a_{m}<\bar{\delta}
$$

ferner $\tilde{i}_{2}$ so, dass es (125) und ausserdem der Bedingung

$$
\left|\tilde{z}_{2}-a_{m} . i^{\dagger}\right|<e^{-G}
$$

genügt. Nach (120), (123), (124) ist tür alle einzelnen Glieder der rechten Seite von (II6) die Beziehung (III) erfullt, sie haben also nach (II4) positive Realteile. Also ist nach (I16) und ( 127 )

$$
\begin{aligned}
& \left|\frac{f\left(z_{2}\right)-f\left(z_{1}\right)}{z_{2}-\tilde{z}_{1}}\right| \geq \Re\left(\frac{f\left(z_{2}\right)-f\left(z_{1}\right)}{z_{2}-z_{1}}\right) \\
& =\sum_{n=1}^{N} \Re\left(\frac{g_{n}\left(z_{2}\right)-g_{n}\left(z_{1}\right)}{i_{2}-z_{1}}+2 b_{n}\right)+\sum_{n=N_{+1}}^{\infty} \sum_{\psi=1}^{j} \Re\left(\frac{b_{n \psi}\left(z_{2}\right)-b_{n \downarrow}\left(z_{1}\right)}{z_{2}-z_{1}}\right)+\sum_{n=\Lambda+1}^{\infty} 2 b_{n} \\
& >\Re\left(\frac{b_{m \varphi}\left(z_{2}\right)-b_{m \varphi}\left(z_{1}\right)}{z_{2}-z_{1}}\right)=\log \left|z_{2}-a_{m} \cdot i^{\varphi}\right|^{-1}>G \text {. }
\end{aligned}
$$

Also gilt (I 26) für beliebig kleine Wertepaare $z_{2}$ und $z_{1}$. Es existiert mithin kein erweiterter absoluter Differentialquotient.

\section{Zweiter Teil.}

Satz II. - Eine eindeutige Funktion einer komplexen Variablen, die an allen Punkten eines einfach zusammenbängenden, abgescblossenen Gebietes $T$ einen von o verschiedenen erweiterten absoluten Differentialquotienten besitzt, ist entweder eine analytische Funktion oder die konjugierte einer analytischen Funktion.

Hilfssatz II. - Besitzt eine eindeutige Funktion $f(z)$ einer komplexen Variablen $z$ ïberall in einem abgescblossenen, einfach zusammenbängenden Gebiete $T$ einen erweiterten absoluten Differentialquotienten, so ist der erweiterte absolute Differentialquotient im Gebiete $T$ eine stetige Funktion 'on $₹$ und existiert gleICHMässig. 
Nach Annahme einer heliebig kleinen reellen positiven Grösse $\varepsilon$ ist $\delta=\delta(\xi, z)$ reell und positiv so bestimmbar, dass, wenn

ist,

(130)

$$
\left|z_{2}-z\right|<\delta
$$

$$
|| \frac{f\left(i_{2}\right)-f\left(i_{1}\right)}{z_{2}-i_{1}}|-m(z)|<\varepsilon
$$

wird. Hierin ist $m(\tilde{z})$ eine Funktion von $\tilde{z}$, die nur reelle nicht negative Werte annimmt. Es sei $\bar{\delta}(\varepsilon, z)$ die obere Grenze der für $\delta(\varepsilon, z)$ zulässigen Werte. Es genüge $z^{\prime}$ der Bedingung

ferner sei

$$
\left|z^{\prime}-z\right|<\bar{\delta}\left(\frac{\varepsilon}{2}, z\right)
$$

$$
\begin{aligned}
& \left|z_{1}-z^{\prime}\right|<\bar{\delta}\left(\frac{\varepsilon}{2}, z\right)-\left|z^{\prime}-z\right|, \\
& \left|z_{2}-z^{\prime}\right|<\bar{\delta}\left(\frac{\varepsilon}{2}, z\right)-\left|z^{\prime}-z\right|
\end{aligned}
$$

und

$$
\begin{aligned}
& \left|z_{1}-z^{\prime}\right|<\bar{\delta}\left(\frac{\varepsilon}{2}, i^{\prime}\right), \\
& \left|z_{2}-z^{\prime}\right|<\bar{\delta}\left(\frac{\varepsilon}{2}, \tilde{i}^{\prime}\right) .
\end{aligned}
$$

Aus ( 13 I) folgt

(I35) $\left|\tilde{i}_{1}-z\right|=\left|\left(\tilde{i}_{1}-\tilde{i}^{\prime}\right)+\left(\tilde{i}^{\prime}-i\right)\right| \leq\left|\tilde{i}_{1}-z^{\prime}\right|+\left|z^{\prime}-i\right|<\delta\left(\frac{\varepsilon}{2}, z\right)$, ebenso

$$
\left|z_{2}-z\right|<\delta\left(\frac{\varepsilon}{2}, \tilde{\imath}\right)
$$

Dann ist wegen (1 35), (136), ( 133 ), ( 134 ), (1 30)

$$
\begin{gathered}
\left|m(z)-m\left(\tilde{i}^{\prime}\right)\right|=\left|\left(m(z)-\left|\frac{f\left(\tilde{z}_{2}\right)-f\left(z_{1}\right)}{z_{2}-z_{1}}\right|\right)-\left(m\left(\tilde{i}^{\prime}\right)-\left|\frac{f\left(\tilde{z}_{2}\right)-f\left(\tilde{z}_{1}\right)}{z_{2}-z_{1}}\right|\right)\right| \\
\leq|| \frac{f\left(z_{2}\right)-f\left(z_{1}\right)}{z_{2}-z_{1}}|-m(i)|+|| \frac{f\left(z_{2}\right)-f\left(\tilde{z}_{1}\right)}{z_{2}-z_{1}}\left|-m\left(i^{\prime}\right)\right|<\frac{\varepsilon}{2}+\frac{\varepsilon}{2}=\varepsilon .
\end{gathered}
$$

Also ist $m(z)$ eine stetige Funktion von $z$.

Es soll gezeigt werden, dass für alle $z$ in $T$ sich

$$
0<\delta(\varepsilon)<\bar{\delta}(\varepsilon, z)
$$

bestimmen lässt. Es sei auf Grund der soeben bewiesenen Stetigkeit von $m(i)$, $z^{\prime}$ so nahe bei $z$ bestimmt, dass

$$
\left|m\left(z^{\prime}\right)-m(z)\right|<\frac{\varepsilon}{2} .
$$

Ferner betrachte man alle $z_{1}$ und $z_{2}$ die den Bedingungen ( 131 ) und (132) genügen, 
aus denen (135) und ( 136$)$ folgen. Es soll gezeigt werden, dass für sie auch (133) und $(134)$ erfültt sind, allerdings nur für $\varepsilon$, nicht für $-\frac{\varepsilon}{2}$. Es ist nämlich nach ( $\left.1 ; 0\right)$ und $(137)$

$$
\begin{gathered}
|| \frac{f\left(z_{2}\right)-f\left(z_{2}\right)}{z_{2}-z_{1}}\left|-m\left(z^{\prime}\right)\right|=\left|\left(\mid \frac{f\left(z_{2}\right)-f(z)}{z_{2}-z_{1}}-m(z)\right)+\left(m(z)-m\left(z^{\prime}\right)\right)\right| \\
\quad \leq|| \frac{f\left(z_{2}\right)-f\left(z_{1}\right)}{z_{2}-z_{1}}|-m(\tilde{z})|+\left|m(\tilde{z})-m\left(z^{\prime}\right)\right|<\frac{\varepsilon}{2}+\frac{\varepsilon}{2}=\varepsilon .
\end{gathered}
$$

Da dies für alle $z_{1}$ und $z_{2}$ gilt, die (131) und (132) genugen, so folgt daraus

$$
\bar{\delta}\left(\frac{\varepsilon}{2}, z\right)-\mid z^{\prime}-\tilde{z}^{\prime} \leq \bar{\delta}\left(\varepsilon, \tilde{i}^{\prime}\right) \text {. }
$$

Diese Ungleichheitsbeziehung gilt erst recht in dem trivialen Falle, dass die linke Seite $\leq \mathrm{o}$. Es sei $k\left(\varepsilon, z^{\prime}\right)$ die obere Grenze aller Werte, die

$$
\bar{\delta}\left(\frac{\varepsilon}{2}, z\right)-\left|z^{\prime}-z\right|
$$

an einer Stelle $z^{\prime}$ für alle Werte von $z$ annimmt. Es gibr also nach Annahme einer beliebig hleinen reellen positiven Grösse $\stackrel{\xi}{3}$ immer einen Wert $z$ derart, dass

$$
\bar{\delta}\left(\frac{\varepsilon}{2}, z\right)-\left|\vec{i}^{\circ}-z\right|>k\left(\varepsilon, z^{\prime}\right)-\bar{\xi} .
$$

Wenn $z$ " ein anderer fester Punkt, wenn ferner $z$ der Bedingung ( 139 ) genügt, so ist

Also ist

$$
\begin{aligned}
\bar{\delta}\left(\frac{\varepsilon}{2}, z\right)-\mid z^{\prime \prime}-z^{\prime} & \geqslant \bar{\delta}\left(\frac{\varepsilon}{2}, z\right)-\left|k^{\prime}-z\right|-\left|z^{\prime \prime}-z^{\prime}\right| \\
& >k\left(\varepsilon, z^{\prime}\right)-\xi-\left|z^{\prime \prime}-z^{\prime}\right| .
\end{aligned}
$$

oder

$$
k\left(\varepsilon, z^{\prime \prime}\right) \supseteq k\left(\varepsilon, z^{\prime}\right)-\left|z^{\prime \prime}-z^{\prime}\right|
$$

$$
k\left(\varepsilon, z^{\prime}\right)-k\left(\varepsilon, z^{\prime \prime}\right) \leq\left|R^{\prime \prime}-z\right| \text {. }
$$

Hierin kann man $z^{\prime}$ und $z^{\prime \prime}$ vertauschen. Also ist

$$
\left|k\left(\varepsilon, z^{\prime}\right)-k\left(\varepsilon, z^{\prime \prime}\right)\right| \leq\left|z^{\prime \prime}-i^{\prime \prime}\right|
$$

Daraus folgt aber die Stetigkeit von $k\left(\varepsilon, z^{\prime}\right)$. Also besitzt $k\left(\varepsilon, z^{\prime}\right)$ in $T$ ein Minimum $k(\varepsilon)$. Da für alle $z^{\prime}$

$$
k\left(\varepsilon, z^{\prime}\right) \geqslant \bar{\delta}\left(\frac{\Sigma}{2}, z^{\prime}\right)>0,
$$

so nuuss das Minimum $k(s)>0$ sein. Es ist aber nach $\left(13^{8}\right)$

$$
\bar{\delta}\left(\varepsilon, z^{\prime}\right) \supseteq k\left(\varepsilon, z^{\prime}\right) \geqq k(\varepsilon)>0 \text {. }
$$

Also lässt sich zu jedem $\varepsilon$ ein von $z$ unablängiges $\delta(\varepsilon)<k(\varepsilon)$ bestimmen, so dass aus dem Bestehen von (128) und (129) das Bestehen von (130) folgt. Es ist somit 
gezeigt worden, dass der erweiterte absolute Differentialquotient gleichmässig im Gebiete $T$ existiert ${ }^{2}$ ).

Ich gehe nun zum Beweise des Satzes (II) über. Es soll gezeigt werden, dass entureder das Integral uber $f(\tilde{z})$ oder das Integral über die konjugierte Funktion $\bar{f}(z)$ längs des Umfanges eines beliebigen geschlossenen, aus geraden Stücken bestehenden Weges gleich o ist, woraus nach dem verallgemeinerten MOrERA'schen Satze ${ }^{3}$ ) folgen wird, dass die Funktion eine analytische ist. Hierzu reicht es wieder hin, zu zeigen, dass das Integral über den Umfang eines beliebigen Dreiecks o ist. Es soll also gezeigt werden, dass

(I 40) $\quad I_{1}+I_{2}+I_{3} \equiv \int_{a_{2}}^{a_{3}} f(z) d z+\int_{a_{2}}^{a_{1}} f(z) d z+\int_{a_{1}}^{a_{2}} f(z) d z=0$.

Hierin sind die Integrale über den geraden Weg zu nehmen. Es sei

$$
A_{1}=\left|a_{3}-a_{2}\right|, \quad A_{2}=\left|a_{1}-a_{3}\right|, \quad A_{3}=\left|a_{2}-a_{1}\right|,
$$

und $z$ war sei die Bezeichnung so gewählt, dass

$$
A_{1} \supseteq A_{2} \supseteq A_{3} \text {. }
$$

Die Gleichung ( 140 ) ist eine Identität, wenn die drei Punkte $a_{1}, a_{2}, a_{3}$, auf einer geraden Linie liegen. Dieser Fall soll ausgeschlossen werden. Dann sind, wie in den Elementen der Theorie der analytischen Funktionen gezeigt wird, sämtliche Faktoren des Produktes

$$
\begin{aligned}
D\left(A_{1}, A_{2}, A_{3}\right) & =\left(A_{1}+A_{2}+A_{3}\right)\left(-A_{1}+A_{2}+A_{3}\right)\left(A_{1}-A_{2}+A_{3}\right)\left(A_{\mathrm{r}}+A_{2}-A_{;}\right) \\
& =2 A_{2}^{2} A_{3}^{2}+2 A_{;}^{2} A_{1}^{2}+2 A_{1}^{2} A_{2}^{2}-A_{1}^{+}-A_{2}^{4}-A_{;}^{4}
\end{aligned}
$$

positiv. Ich will der Kürze halber bei der Funktion $D$ die Argumente $A_{1}, A_{2}, A_{\text {; }}$ und nur diese, fortlassen.

Es sei ferner $M$ das Maximum von $m(\tilde{i})$ im Gebiete $T$, das existieren muss, da nach dem Hilfssatze (II) $m(\tilde{z})$ in $T$ stetig ist, ebenso $m$ das Minimum, das nach der gemachten V'oraussetzung von o verschieden sein muss. Es sei ferner die beliebig kleine reelle positive Zahl $₹$ vorgegehen, die der Bedingung genüge

$$
\varepsilon<\frac{\mathrm{I}}{3} M 1^{\prime} \bar{D}
$$

Man bestimme die pusitive ganze Zahl $n$ so gross, dass

$$
n>\frac{3 A_{1}^{2}(M+m \sigma)}{\varepsilon}
$$

wo $\approx$ den in $(\mathrm{I}+5)$ angegebenen Ausdruik bedeute. Ferner bestimme man gleichmässig

2) Aehnlich liesse sich auch der Satz beweisen. dass eine stetige Funktion gleichmàssig stetig ist. Andererseits lässt sich auch dieser Beweis hier in der bchannten Weise indircht fuhren durch fortgesetzte Unterteilung des Gebietes, die auf einen Punht tuhrt, an dem ein W"iderspruch statthindet.

3) G. SORERA, Un teviema fondamentale nella teorica delle iuntioni di una variabile complessa [Rentdiconti del R. Istituto Lombardo di Sienze e Lettere (Milano), Ser. II, Bd. XIX (1886), S. 304-307]. Uiber die Verallgemeinerung siehe: W. F. OsGoov, Funktionentheorie, 2. Aull. 1912, S. 302. 
für alle $z, n$ so gross, dass für

$$
\left.\begin{array}{l}
z_{1}-z \\
z_{2}-z
\end{array}\right\}<\frac{A_{1}}{n}
$$

$$
|| \frac{f\left(z_{2}\right)-f\left(z_{1}\right)}{z_{2}-z_{1}}|-m(z)|<m . \sigma \equiv m\left|\frac{\int_{4} A_{1}^{2} M+\frac{\sqrt{D}}{A^{2}} \cdot \varepsilon}{\mid \sqrt{16 A_{1}^{+} M^{2}}+\frac{1}{9 \varepsilon^{2}}}-1\right|
$$

wird, woraus folgt

$(1+6) \quad\left|f\left(z_{2}\right)-f\left(z_{1}\right)\right|<\left|z_{2}-z_{1}\right| \cdot(m(z)+m . \sigma) \leq\left|z_{2}-z_{1}\right| \cdot(M+m . \sigma)$.

Un zu zeigen, dass $\sigma$ eine positive Grösse ist, setze man

( 147 )

$$
(\mathrm{I}+\sigma)^{2}=\mathrm{I}+\bar{\sigma} .
$$

Dann ist nach $(\mathbf{I} 43)$

$$
\bar{\sigma}=\frac{8 M \sqrt{D} \varepsilon+\frac{D}{A_{1}^{4}} \varepsilon^{2}-9 \varepsilon^{2}}{16 A_{1}^{+} M^{2}+9 \varepsilon^{2}}>\frac{5 M V^{\prime} \bar{D} \varepsilon+\frac{D}{A_{1}^{+}} \varepsilon^{2}}{\mathrm{I} 6 A_{1}^{+}}>0,
$$

also ist auch

$$
(\mathrm{I}+\sigma)^{2}>\mathrm{I}, \quad \mathrm{I}+\sigma>\mathrm{I}, \sigma>0
$$

Man führe zur Abkürzung die Bezeichnung ein

$$
\frac{\alpha a_{1}+\beta a_{2}+\gamma a_{3}}{n}=b_{a, \bar{\gamma},},
$$

worin $\alpha, \beta, \gamma$ positive ganze Zahlen, die der Bedingung genügen

ganz ebenso

$$
\alpha+\beta+\gamma=n
$$

$$
\frac{\alpha a_{1}+\beta a_{2}+\gamma a_{i}}{2 n}=c_{\alpha \dot{r} \gamma} \text {, }
$$

worin

Es ist

$$
\alpha+\beta+\gamma=2 n \text {. }
$$

Ferner ist

$$
\begin{gathered}
c_{2 x, 2 \beta, 2 Y}=b_{\alpha \beta \gamma}, \\
c_{2 \alpha+1,2 \beta-1,2 Y}=\frac{b_{x \not Y}+b_{\alpha+1, \beta-1, \gamma}}{2} .
\end{gathered}
$$

$(1+9)$

$$
\left\{\begin{array}{l}
b_{\lambda, \mu, \nu+1}-b_{\lambda, \mu+1, \nu}=\frac{a_{3}-a_{2}}{n}, \\
b_{\lambda+1, \mu, \nu}-b_{\lambda, \mu, \nu+1}=\frac{a_{1}-a_{3}}{n}, \\
b_{\lambda, \mu+1, \nu}-b_{\lambda+1, \mu, \nu}=\frac{a_{2}-a_{1}}{n}
\end{array}\right.
$$

und entsprechend für die $i_{x \neq \gamma}$. Unter Benutzung von $(1+9),(1+6),(1+2),(1+4)$ erhält 
man

(150)

$$
\begin{aligned}
\mid I_{1}+I_{2}+I_{i} & -\frac{a_{3}-a_{2}}{n} \cdot \sum_{v=1}^{n} f\left(c_{0,2 n-2 v+1,2 v-1}\right) \\
& -\frac{a_{1}-a_{3}}{n} \cdot \sum_{v=1}^{n} f\left(c_{2 v-1,0,2 n-2 v+1}\right)-\frac{a_{2}-a_{1}}{2} \cdot \sum_{v=1}^{n} f\left(c_{2 n-2 v+1,2 v-1,0}\right) \mid \\
& \equiv\left|I_{1}+I_{2}+I_{3}-S_{1}-S_{2}-S_{3}\right| \\
& =\mid \sum_{v=1}^{n} \int_{b_{0, n-v+1, v-1}}^{h_{0, n-v, v}}\left(f(z)-f\left(c_{0,2 n-2 v+1,2 v-1}\right)\right) d z \\
& +\sum_{v=1}^{n} \int_{b_{v-1,0, n-v+1}}^{b_{v, 0, n-v}}\left(f(z)-f\left(c_{2 v-1,0,2 n-2 v+1}\right)\right) d z \\
& +\sum_{v=1}^{n} \int_{b_{n-v+1, v-1,0}}^{h_{n-v, v, 0}}\left(f(z)-f\left(c_{2 n-2 v+1,2 v-1,0}\right)\right) d z \mid \\
& <3 n \cdot \frac{A_{1}}{n} \cdot \frac{A_{1}}{2 n}(M+m . \sigma)<\frac{\varepsilon}{2} .
\end{aligned}
$$

Es gilt die Identität

$$
\mid \begin{aligned}
& \sum_{I}+\sum_{I I} \equiv \sum_{\substack{\lambda=0, \mu=0, \nu=0 \\
\lambda+\mu+\nu=n-1}}^{n=1}\left\{\left(b_{\lambda, \mu, \nu+1}-b_{\lambda, \mu+1, \nu}\right) \cdot f\left(c_{2 \lambda, 2 \mu+1,2 \nu+1}\right)\right. \\
& \left.+\left(b_{\lambda+1, \mu, \nu}-b_{\lambda, \mu, \nu+1}\right) \cdot f\left(c_{2 \lambda+1,2 \mu, 2 \nu+1}\right)+\left(b_{\lambda, \mu+1, \nu}-b_{\lambda+1, \mu, \nu}\right) \cdot f\left(c_{2 \lambda+1,2 \mu+1,2 \nu}\right)\right\} \\
& +\sum_{\substack{\lambda=0, \mu=0, \nu=0 \\
\lambda+\mu+\nu=12=2}}^{n=2}\left\{\left(b_{\lambda+1, \mu+1, \nu}-b_{\lambda+1, \mu, \nu+1}\right) \cdot f\left(c_{2 \lambda+2,2 \mu+1,2 \nu+1}\right)\right.
\end{aligned}
$$

(I I I)

$$
\left.+\left(b_{\lambda, \mu+1, \nu+1}-b_{\lambda+1, \mu+1, \nu}\right) \cdot f\left(c_{2 \lambda+1,2 \mu+2,2 v+1}\right)+\left(b_{\lambda+1, \mu, \nu+1}-b_{\lambda, \mu+1, \nu+1}\right) \cdot f\left(c_{2 \lambda+1,2 \mu+1,2 \nu+2}\right)\right\}
$$

$$
\left\{\begin{array}{l}
=\sum_{\substack{\mu=0, v=0 \\
\mu+\nu=n-1}}^{n=1}\left(b_{o, \mu, \nu+1}-b_{0, \mu+1, \nu}\right) \cdot f\left(c_{0,2 \mu+1,2 v+1}\right) \\
+\sum_{\substack{\lambda=0, \nu=0 \\
\lambda+\nu=n-1}}^{n-1}\left(b_{\lambda+1,0, v}-b_{\lambda, 0, \nu+1}\right) \cdot f\left(c_{2 \lambda+1,0,2 \nu+1}\right) \\
+\sum_{\substack{\lambda=0, \mu=0 \\
\lambda+\mu=n-1}}^{n=1}\left(b_{\lambda, \mu+1,0}-b_{\lambda+1, \mu, 0}\right) \cdot f\left(c_{2 \lambda+1,2 \mu+1,0}\right)=S_{1}+S_{2}+S_{3} .
\end{array}\right.
$$

Die erste Summe $\sum_{l}$ auf der linken Seite enthält $\frac{n(n+1)}{2}$, die zweite $\sum_{I}$ enthält $\frac{n(n-\mathrm{I})}{2}$ Glieder; zusammen sind es also $n^{2}$ Glieder von der Form

$$
S\left(f(z) ; b_{1}, b_{2}, b_{3}\right)=\left(b_{3}-b_{2}\right) \cdot f\left(c_{1}\right)+\left(b_{1}-b_{3}\right) \cdot f\left(c_{2}\right)+\left(b_{2}-b_{1}\right) \cdot f\left(c_{3}\right),
$$

wo

Es ist ferner

$$
c_{1}=\frac{b_{2}+b_{3}}{2}, \quad c_{2}=\frac{b_{3}+b_{1}}{2}, \quad c_{3}=\frac{b_{1}+b_{2}}{2} .
$$

$$
\frac{A_{1}}{n}=\left|b_{3}-b_{2}\right|, \quad \frac{A_{2}}{n}=\left|b_{1}-b_{3}\right|, \quad \frac{A_{3}}{n}=\left|b_{2}-b_{1}\right|
$$

(I52) $c_{j}-c_{2}=-\frac{b_{3}-b_{2}}{2}, \quad c_{1}-c_{j}=-\frac{b_{1}-b_{3}}{2}, \quad c_{2}-c_{1}=-\frac{b_{2}-b_{1}}{2}$. 
Man setze

$(153)$

$$
\left\{\begin{array}{l}
\left|f\left(c_{3}\right)-f\left(c_{2}\right)\right|=\left|c_{3}-c_{2}\right| \cdot m\left(c_{1}\right) \cdot\left(1+n_{1}\right), \\
\left|f\left(c_{1}\right)-f\left(c_{3}\right)\right|=\left|c_{1}-c_{3}\right| \cdot m\left(c_{3}\right) \cdot\left(1+n_{2}\right), \\
\left|f\left(c_{2}\right)-f\left(c_{1}\right)\right|=\left|c_{2}-c_{1}\right| \cdot m\left(c_{3}\right) \cdot\left(1+n_{3}\right),
\end{array}\right.
$$

dann ist nach $(1+5)$

$$
\left|\begin{array}{l}
\left|n_{1}\right| \\
\left|n_{3}\right|
\end{array}\right|<\frac{m \cdot \sigma}{m\left(c_{3}\right)} \leq \sigma
$$

Es ist

$$
\left\{\begin{array}{c}
S\left(f(z) ; b_{1}, b_{2}, b_{3}\right)=\left(b_{3}-b_{2}\right)\left(f\left(c_{1}\right)-f\left(c_{2}\right)\right)+\left(b_{2}-b_{1}\right)\left(f\left(c_{3}\right)-\left(c_{2}\right)\right) \\
=\left(f\left(c_{3}\right)-f\left(i_{3}\right)\right)\left(b_{;}-b_{2}\right) \cdot \frac{\mid f\left(c_{1}\right)-f\left(c_{2}\right)}{\mid f\left(c_{3}\right)-f\left(c_{2}\right)}-\frac{b_{1}-b_{2} \mid}{b_{3}-b_{2} \mid} .
\end{array}\right.
$$

Man setze

$$
\begin{aligned}
& \left|\hat{c}_{3}-c_{2}\right|=e_{1}, \quad\left|f\left(c_{3}\right)-f\left(c_{2}\right)\right|=E_{1}, \\
& \left\{\left|c_{1}-c_{j}\right|=c_{2}, \quad\left|f\left(c_{1}\right)-f\left(c_{3}\right)\right|=E_{2},\right.
\end{aligned}
$$

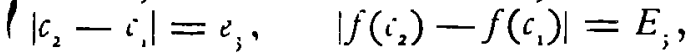

ferner

$(15 \overline{1})$

$$
\frac{b_{1}-b_{2}}{b_{3}-b_{2}}=\frac{c_{1}-c_{2}}{c_{3}-c_{2}}=x_{2}, \quad \frac{f\left(c_{1}\right)-f\left(c_{2}\right)}{f\left(c_{3}\right)-f\left(c_{2}\right)}=X_{2} .
$$

Es gelten die Gleichungen

es ist also

$$
\left|x_{2}\right|=\frac{e_{3}}{e_{1}}, \quad\left|x_{2}-1\right|=\frac{\mid c_{1}-c_{3}}{\mid c_{3}-c_{2}} \mid=\frac{c_{2}}{e_{1}} ;
$$

$$
\left\{\begin{array}{l}
\Re\left(x_{2}\right)=\frac{\mathrm{I}}{2}\left\{x_{2} \bar{x}_{2}+\mathrm{I}-\left(x_{2} \bar{x}_{2}-x_{2}-\bar{x}_{2}+\mathrm{I}\right)\right\} \\
=\frac{\mathrm{I}}{2}\left(\left|x_{2}\right|^{2}+\mathrm{I}-\left|x_{2}-\mathrm{I}\right|^{2}\right)=\frac{e_{3}^{2}+e_{1}^{2}-e_{2}^{2}}{2 e_{1}^{2}} \equiv \frac{q_{2}}{2 e_{1}^{2}},
\end{array}\right.
$$

worin $q_{2}$ zur Abkürzung eingeführt ist. Hieraus ergibt sich

( I60)

$$
\left\{\begin{array}{c}
\mathfrak{S}^{2}\left(x_{2}\right)=\left|x_{2}\right|^{2}-\mathfrak{R}^{2}\left(x_{2}\right)=\frac{4 e_{1}^{2} e_{3}^{2}-q_{2}^{2}}{4 e_{1}^{4}} \\
=\frac{\left(e_{1}+e_{2}+e_{3}\right)\left(-e_{1}+e_{2}+e_{3}\right)\left(e_{1}-e_{2}+e_{3}\right)\left(e_{1}+e_{2}-e_{3}\right)}{4 e_{1}^{4}}=\frac{D\left(e_{1}, e_{2}, e_{3}\right)}{4 e_{1}^{4}} .
\end{array}\right.
$$

Eebenso erhält man

$$
\begin{gathered}
\Re\left(X_{2}\right)=\frac{E_{2}^{2}+E_{1}^{2}-E_{2}^{2}}{2 E_{1}^{2}}=\frac{Q_{2}}{2 E_{1}^{2}}, \\
\mathfrak{J}^{2}\left(X_{2}\right)=\frac{D\left(E_{1}, E_{2}, E_{3}\right)}{4 E_{1}^{4}} .
\end{gathered}
$$

Benutzt man ( 152 ) und setzt man ( 156$),(157)$, ( 159$),(160)$ in (155) ein, so erhält 
man

(162)

$$
\begin{aligned}
& \left.|| S\left(f(i) ; b_{1}, b_{2}, b_{3}\right)\right|^{2}=4 e_{1}^{2} E_{1}^{2}\left\{\left(\Re\left(X_{2}\right)-\Re\left(x_{2}\right)\right)^{2}+\left(\mathfrak{I}\left(X_{2}\right)-\mathfrak{J}\left(x_{2}\right)\right)^{2}\right\} \\
& =4 e_{1}^{2} E_{1}^{2}\left\{\left|X_{2}\right|^{2}+\left|x_{2}\right|^{2}-2 \Re\left(X_{2}\right) \cdot \Re\left(x_{2}\right)-2 \mathfrak{\Im}\left(X_{2}\right) \cdot \mathfrak{\Im}\left(x_{2}\right)\right\} \\
& ={ }_{4} E_{3}^{2} e_{1}^{2}+{ }_{4} E_{1}^{2} e_{3}^{2}-2 Q_{2} q_{2}-21 \overline{D(} \overline{\left.E_{1}, E_{2}, E_{3}\right)} \cdot 1^{\prime} \overline{D\left(e_{1}, e_{2}, e_{3}\right)} \\
& =2\left(E_{j}^{2} e_{1}^{2}+E_{1}^{2} e_{j}^{2}+E_{1}^{2} e_{2}^{2}+E_{2}^{2} e_{1}^{2}+E_{2}^{2} e_{3}^{2}+E_{j}^{2} e_{2}^{2}-E_{1}^{2} e_{1}^{2}-E_{2}^{2} e_{2}^{2}-E_{3}^{2} e_{j}^{3}\right) \\
& -2 \sqrt{D\left(E_{1}, E_{2}, E_{3}\right) \cdot D\left(e_{1}, e_{2}, e_{3}\right)} \\
& \equiv 2 \Delta\left(E_{1}, E_{2}, E_{;} ; e_{1}, e_{2}, e_{j}\right)-2 \downarrow^{\prime} \overline{D\left(E_{1}, E_{2}, E_{3}\right) \cdot D\left(e_{1}, e_{2}, e_{3}\right)}
\end{aligned}
$$

wo $د\left(E_{1}, E_{2}, E_{;} ; e_{1}, e_{2}, e_{3}\right)$ zur Abkürzung eingeführt ist. Betrachtet man anstatt $f(z)$ die konjugierte Funktion $\bar{f}(z)$, so ändert sich nur das Vorzeichen von $\mathfrak{F}\left(X_{2}\right)$. Es sei also unter den beiden konjugierten Funktionen $f(z)$ und $\bar{f}(z)$ diejenige ausgewählt, fur welche die Vorzeichen von $\mathfrak{i}\left(X_{2}\right)$ und $i\left(x_{2}\right)$ ubereinstimmen. Dass dieser Bedingung gleichzeitig für alle $n^{2}$ Dreiecke $b_{1}, b_{2}, b_{\text {; }}$ genügt werden kann, soll weiter unten gezeigt werden. Es ist also die Quadratwurzel im letzten Ausdrucke von ( 162 ) eine positive Grösse. Es ergibt sich weiter

$$
\left\{=\frac{د^{2}\left(E_{1}, E_{2}, E_{j} ; e_{1}, e_{2}, e_{i}\right)-D\left(E_{1}, E_{2}, E_{3}\right) \cdot D\left(e_{1}, e_{2}, e_{3}\right)}{د\left(E_{1}, E_{2}, E_{3} ; e_{1}, e_{2}, e_{j}\right)+1^{\prime} \overline{D\left(E_{1}, E_{2}, E_{3}\right) \cdot D\left(e_{1}, e_{2}, e_{3}\right)}} .\right.
$$

Es wird nach (160) und (162)

$$
\begin{aligned}
& J^{2}\left(E_{1}, E_{2}, E_{j} ; e_{1}, e_{2}, e_{3}\right)-D\left(E_{1}, E_{2}, E_{3}\right) \cdot D\left(e_{1}, e_{2}, e_{3}\right) \\
& \quad=4\left(E_{3}^{2} e_{1}^{2}+E_{1}^{2} e_{3}^{2}\right)^{2}-4\left(E_{j}^{2} e_{1}^{2}+E_{1}^{2} e_{3}^{2}\right) Q_{2} q_{2}+Q_{2}^{2} q_{2}^{2} \\
& \quad-16 E_{1}^{2} E_{3}^{2} e_{1}^{2} e_{3}^{2}+{ }_{4} E_{1}^{2} E_{3}^{2} q_{2}^{2}+4 e_{1}^{2} e_{3}^{2} Q_{2}^{2}-Q_{2}^{2} q_{2}^{2} \\
& =4\left(Q_{2} e_{1}^{2}-q_{2} E_{1}^{2}\right)\left(Q_{2} e_{3}^{2}-q_{2} E_{j}^{2}\right)+{ }_{4}\left(E_{3}^{2} e_{1}^{2}-E_{1}^{2} e_{3}^{2}\right)^{2} .
\end{aligned}
$$

Setzt man ferner zur Abkürzung

$$
\left(1+n_{1}\right)^{2}=1+\bar{n}_{1}, \quad\left(1+n_{2}\right)^{2}=1+\bar{n}_{2}, \quad\left(1+n_{3}\right)^{2}=1+\bar{n}_{3},
$$

woraus in Verbindung mit (147) und (154) folgt,

und setzt man

$$
\frac{\left|n_{1}\right|}{\left|n_{2}\right|} \mid<\bar{\sigma}
$$

$$
\left\{\begin{array}{l}
R_{2} \equiv E_{3}^{2} e_{1}^{2}-E_{1}^{2} e_{3}^{2}=m^{2}\left(c_{3}\right) \cdot e_{3}^{2} e_{3}^{2}\left(\bar{n}_{3}-\overline{n_{1}}\right) \equiv m^{2}\left(c_{3}\right) \cdot e_{1}^{2} e_{3}^{2} \bar{\xi}_{2} \\
R_{3} \equiv E_{1}^{2} e_{2}^{2}-E_{2}^{2} e_{1}^{2}=m^{2}\left(c_{3}\right) \cdot e_{2}^{2} e_{1}^{2}\left(\overline{n_{1}}-\overline{n_{2}}\right) \equiv m^{2}\left(c_{3}\right) \cdot e_{2}^{2} e_{1}^{2} \bar{\xi}_{3} \\
R_{1} \equiv E_{2}^{2} e_{3}^{2}-E_{3}^{2} e_{2}^{2}=m^{2}\left(c_{3}\right) \cdot e_{3}^{2} e_{2}^{2}\left(\overline{n_{2}}-\overline{n_{3}}\right) \equiv m^{2}\left(c_{3}\right) \cdot e_{3}^{2} e_{2}^{2} \bar{\xi}_{1}
\end{array}\right.
$$

so ergibt sich

$$
\text { (I66) }\left\{\begin{array}{l}
\mathrm{J}^{2}\left(E_{1}, E_{2}, E_{3} ; e_{1}, e_{2}, e_{3}\right)-D\left(E_{1}, E_{2}, E_{3}\right) \cdot D\left(e_{1}, e_{2}, e_{3}\right) \\
=4\left(R_{2}+R_{3}\right)\left(-R_{2}-R_{1}\right)+4 R_{2}^{2} \\
=-4\left(R_{2} R_{3}+R_{3} R_{1}+R_{1} R_{2}\right)=-4 e_{2}^{2} e_{2}^{2} e_{3}^{2} \cdot m^{4}\left(c_{3}\right) \cdot\left(e_{1}^{2} \bar{\xi}_{2} \bar{\xi}_{3}+e_{2}^{2} \bar{\xi}_{3} \bar{\xi}_{1}+e_{3}^{2} \bar{\xi}_{1} \bar{\xi}_{2}\right) .
\end{array}\right.
$$


Es ist nach ( 165 )

$(167)$

$$
\bar{\xi}_{1}+\bar{\xi}_{2}+\bar{\xi}_{3}=0 .
$$

Es mögen $\bar{\xi}_{x}$ und $\bar{\xi}_{p}$ gleiches Yorzeichen besitzen, dann ist

also unter Benutzung von $(164)$

$$
\bar{\xi}_{\gamma}=-\bar{\xi}_{x}-\bar{\xi}_{\beta}
$$

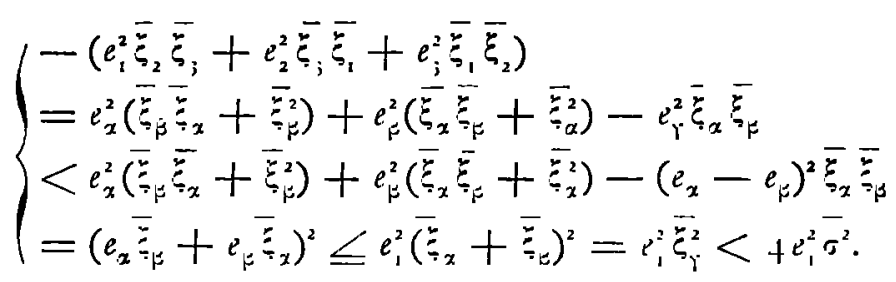

Ferner definiere man entsprechend (159)

$$
q_{1}=e_{2}^{2}+e_{3}^{2}-e_{1}^{2}, \quad q_{3}=e_{1}^{2}+e_{2}^{2}-e_{3}^{2}
$$

dann erhält man

$$
\left\{\begin{array}{l}
\Delta\left(E_{1}, E_{2}, E_{3} ; e_{1}, e_{2}, e_{i}\right) \\
=m^{2}\left(c_{3}\right) \cdot\left\{D\left(e_{1}, e_{2}, e_{j}\right)+\bar{n}_{1} e_{1}^{2} q_{1}+\bar{n}_{2} e_{2}^{2} q_{2}+\bar{n}_{3} e_{3}^{2} q_{3}\right\} \\
>m^{2}\left(c_{3}\right) \cdot\left\{D\left(e_{1}, e_{2}, e_{i}\right)-\bar{\sigma}\left(e_{1}^{2}\left|q_{1}\right|+e_{2}^{2}\left|q_{2}\right|+e_{3}^{2}\left|q_{3}\right|\right)\right\} .
\end{array}\right.
$$

$q_{2}$ und $q_{3}$ sind immer positiv; in Bezug auf das Vorzeichen von $q_{1}$ unterscheide man zwei Fälle:

Fall $(a)$ : Es sei $q_{1} \gg 0$, (das Dreieck $a_{1} a_{2} a_{\text {; }}$ sei spitzwinklig oder rechtwinklig). Dann ist also

$$
D\left(e_{1}, e_{2}, e_{3}\right)=e_{1}^{2} q_{1}+e_{2}^{2} q_{2}+e_{3}^{2} q_{3} \leq e_{1}^{2}\left(q_{1}+q_{2}+q_{3}\right) \leq 3 e_{1}^{+} .
$$

Fall $(b)$ : Es sei $q_{1}<0$, (das Dreieck $a_{1} a_{2} a_{3}$ sei also stumpfwinklig). Dann ist (ITI $)\left\{\begin{array}{l}D\left(e_{1}, e_{2}, e_{3}\right)<e_{1}^{2}\left|q_{1}\right|+e_{2}^{2}\left|q_{2}\right|+e_{3}^{2}\left|q_{3}\right| \\ =e_{1}^{2}\left(e_{1}^{2}-e_{2}^{2}-e_{3}^{2}\right)+e_{2}^{2}\left(e_{1}^{2}+e^{2}-e_{2}^{2}\right)+e_{3}^{2}\left(e_{1}^{2}+e_{2}^{2}-e_{3}^{2}\right)=e_{1}^{4}-\left(e_{2}^{2}-e_{3}^{2}\right)^{2}<3 e_{1}^{4} .\end{array}\right.$

In beiden Fällen erhält man also aus (169)

$$
د\left(E_{1}, E_{2}, E_{3} ; e_{1}, e_{2}, e_{3}\right)>m^{2}\left(c_{3}\right) \cdot\left\{D\left(e_{1}, e_{2}, e_{3}\right)-3 \bar{\sigma} e_{1}^{+}\right\} .
$$

Unter Benutzung derselben Abschätzung erhält man

$$
\left\{\begin{array}{l}
D\left(E_{1}, E_{2}, E_{3}\right)=m^{4}\left(c_{3}\right) \cdot\left\{D\left(e_{1}, e_{2}, e_{3}\right)+2\left(\bar{n}_{1} e_{1}^{2} q_{1}+\bar{n}_{2} e_{2}^{2} q_{2}+\bar{n}_{3} e_{3}^{2} q_{3}\right)\right. \\
\left.+2 \bar{n}_{1} e_{1}^{2} \bar{n}_{2} e_{2}^{2}+2 \bar{n}_{2} e_{2}^{2} \bar{n}_{3} e_{3}^{2}+2 \bar{n}_{3} e_{3}^{2} \bar{n}_{1} e_{1}^{2}-\bar{n}_{1}^{2} e_{1}^{4}-\bar{n}_{2}^{2} e_{2}^{4}-\bar{n}_{3} e_{3}^{4}\right\} \\
>m^{4}\left(c_{3}\right)\left\{D\left(e_{1}, e_{2}, e_{3}\right)-6 \bar{\sigma} e_{1}^{4}-9 \bar{\sigma}^{2} e_{1}^{4}\right\} .
\end{array}\right.
$$

Setzt man (I68) in (I66) und dies gleichzeitig mit (I72) und (I73) in (I63) ein, so 
erhält man

$$
\begin{aligned}
& \left|S\left(f(z) ; b_{1}, b_{2}, b_{;}\right)\right|^{2}
\end{aligned}
$$

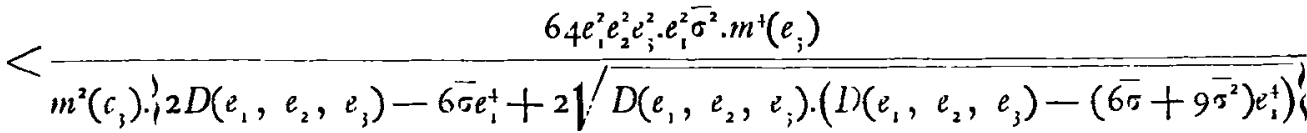

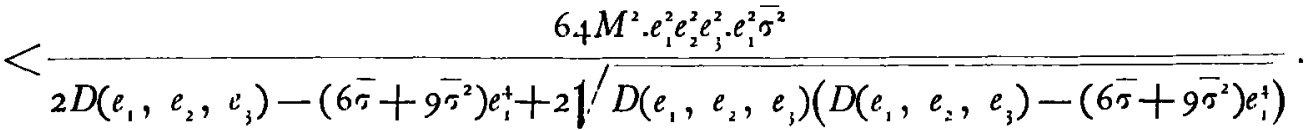

Also ist

$$
(\mathrm{I} 74)\left\{\begin{array}{l}
\left|S\left(f(z) ; b_{1}, b_{2}, b_{j}\right)\right|<\frac{8 M e_{1} e_{2} e_{3} \cdot e_{1} \bar{\sigma}}{1^{\prime} \overline{D\left(e_{1}, e_{2}, e_{j}\right)}+1 \overline{D\left(e_{1}, e_{2}, e_{3}\right)-\left(6 \bar{\sigma}+9 \overline{\sigma^{2}}\right) e_{1}^{+}}} \\
\leq \frac{2 M \cdot A_{1}^{4} \bar{\sigma}}{n^{2}\left(\sqrt{D}+\sqrt{D-\left(6 \bar{\sigma}+9 \bar{\sigma}^{2}\right) A_{1}^{4}}\right)}=\frac{2 M\left(1^{\prime} \bar{D}-\sqrt{D-\left(6 \bar{\sigma}+9 \bar{\sigma}^{2}\right) A_{1}^{+}}\right)}{n^{2}(6+9 \bar{\sigma})} .
\end{array}\right.
$$

Nun folgt aber aus $\left(\mathrm{I} 4^{8}\right)$ in Verbindung mit (I 70$),(\mathrm{I} / \mathrm{I})$ und $(\mathrm{I} / 3)$

$$
\bar{\sigma}<\frac{8 M{ }^{1} \bar{D} \varepsilon-6 \varepsilon^{2}}{16 A_{1}^{4} M^{2}+9 \varepsilon^{2}}<\frac{M 1 \bar{D} \varepsilon}{2 A_{1}^{4} M^{2}}<\frac{M^{2} D}{6 M^{2} A_{!}^{!}} \leq \frac{1}{2} .
$$

Aus dem ersten Teile dieser Ungleichheitsbeziehung erhält man

oder

$$
(6+9 \bar{\sigma}) \varepsilon^{2}-8 M \sqrt{D} \varepsilon<-16 A_{1}^{4} M^{2} \bar{\sigma}
$$

$$
(4 M 1 \bar{D}-(6+9 \bar{\sigma}) s)^{2}<16 M^{2}\left(D-\bar{\sigma}(6+9 \bar{\sigma}) A_{1}^{4}\right)
$$

oder mit Benutzung des letzten Teiles von $(175)$ und von $(1+3)$

$$
\begin{aligned}
& \int{ }_{4} M l^{\prime} \overline{D-\bar{\sigma}(6+9 \bar{\sigma}) A_{1}^{4}}>{ }_{4} M l^{\prime} \bar{D}-(6+9 \bar{\sigma}) \varepsilon \\
& \text { ! }>{ }_{4} M \sqrt{D}-\frac{2 \mathrm{I}}{2} \cdot \frac{\mathrm{I}}{3} M{ }_{1} \bar{D}=\frac{\mathrm{I}}{2} M V^{\prime} \bar{D}>0 \text {. }
\end{aligned}
$$

Die Quadratwurzel in $(176)$ ist also reell. Setzt man den ersten Teil von $\left(17^{6}\right)$ in (I7) ein, so erhält man

$$
\left|S\left(f(z) ; b_{1}, b_{2}, b_{;}\right)\right|<\frac{\varepsilon}{2 n^{2}} .
$$

Es soll jetzt gezeigt werden, dass das Vorzeichen von $3(X)$ für alle $n^{2}$ in Betracht kommenden Dreiecke $b_{1} b_{2} b_{3}$ bezw. $c_{1} c_{2} c_{;}$dasselbe ist. Es sind sämtliche Wertetripel $c_{1} c_{2} c_{3}$ in $\Sigma_{I}$ und $\Sigma_{I I}$ von der Form

$$
z+\frac{\rho a_{1}}{2 n}, \quad z+\frac{\rho a_{2}}{2 n}, \quad z+\frac{\rho a_{3}}{2 n},
$$

wo $₹$ gewisse Werte in Dreieck $a_{1} a_{2} a_{3}$ annimmt und $\hat{p}= \pm 1$. Man lasse für $\tilde{z}$ alle Werte in $T$, für : alle Werte rom absoluten Betrage $I z u$, dann ist $X$ eine stetige Funktion von $z$ und $;$, denn der Nenner von $X$ ist absolut genommen nach (153), 
(154) und der Endabschätzung von $(175)$

$$
\begin{aligned}
& \left|f\left(z+\frac{p \alpha_{3}}{2 n}\right)-f\left(z+\frac{p a_{2}}{2 n}\right)\right|=\left|\frac{p\left(a_{3}-a_{2}\right)}{2 n}\right| \cdot m(z) \cdot\left(1+n_{1}\right) \\
& \doteq \frac{A_{1}}{2 n} \cdot m \cdot(\mathrm{I}-\sigma)=\frac{A_{1}}{2 n} \cdot m\left(2-1^{\prime} \cdot \overline{\mathrm{I}+\bar{\sigma}}\right) \\
& >\frac{A_{1}}{2 n} \cdot m \cdot\left(2-1 / \sqrt{\frac{3}{2}}\right)>\frac{3 A_{1} m}{8 n}>0 .
\end{aligned}
$$

Ferner ist nach $(161),(173)$ und den Endabschätzungen von $(1,6)$ und $(175)$

$$
\begin{aligned}
\hat{j}^{2}(X) & =\frac{D\left(E_{1}, E_{2}, E_{j}\right)}{4 E_{1}^{4}}>\frac{D-\left(6 \bar{\sigma}+9 \bar{\sigma}^{2}\right) A_{1}^{+}}{4 A_{1}^{4}(1+\sigma)^{4}} \\
& >\frac{D}{8^{2} \cdot+A_{1}^{+} \cdot\left(\frac{3}{2}\right)^{2}}=\frac{D}{57^{6} A_{1}^{4}}>0
\end{aligned}
$$

also kann $\mathfrak{J}(X)$ nirgends o werden, also auch nicht das Torzeichen wechseln, also hat $\mathfrak{J}(X)$ in allen Dreiecken $c_{1}, c_{2}, c_{3}$ ein ganz bestimmtes Vorzeichen für $f(z)$ und das entgegengesetzte für $\bar{f}(\tilde{\imath})$. Wählt man diejenige der beiden Funktionen $f(z)$ und $\bar{f}(z)$, für die $\Im(x)$ und $\Im(X)$ gleiches Vorzejihen besitzen, so ist für diese Funktion (I62) erfüllt, also alle folgenden Ungleichheitsbeziehungen, insbesondere (177). Jetzt folgt aber aus (ISI)

$$
\left|S_{1}+S_{2}+S_{j}\right|=\left|\mathbf{\Sigma}_{l}+\mathbf{s}_{l l}\right| \leq n^{2} \cdot \frac{\varepsilon}{2 n^{2}}=\frac{\varepsilon}{2} .
$$

Also ist nach ( 150$)$ und $\left(17^{8}\right)$

$\left|I_{1}+I_{2}+I_{, 1} \leq\right| I_{1}+I_{2}+I_{j}-S_{1}-S_{2}-S_{j}|+| S_{1}+S_{2}+S_{j} \mid<\frac{\varepsilon}{2}+\frac{\varepsilon}{2}=\varepsilon$

Da aber die linke Seite dieser Gleichung als absoluter Betrag eines bestimmten Integrals eine feste Zahl ist, so muss

$$
I_{1}+I_{2}+I_{;}=0
$$

sein. Das Integral über den Umfang eines beliebigen Dreiecks ist o; hieraus folgt aber, wie zu Anfang dieses Teiles der Arbeit erörtert, dass $f(z)$ bezw. $\bar{f}(z)$ eine reguläre analytische Funktion sein muss.

Besitzt $m(z)$ eine isolierte Nullstelle, so ist $f(z)$ bezw. $\bar{f}(i)$ in einer endlichen Umgebung dieser Stelle regülar und an der Stelle selber stetig, also auch an dieser Stelle regulär nach einem bekannten Satze der Funktionentheorie.

Die Voraussetzung dieses Satzes lasst sich etwas anders fassen.

Hilfssatz III. - Besitnt eine eindeutige Funktion $f(\tilde{z})$ einer komplexen Variablen ; in einem einfach zusummenbängenden, atgescblossenen Gebiete $T$ Gleichmässig den grüöbn. licben absoluten Differentialquotienten, so ist dieser eine stetige Funktion von $ぇ$, und f(z) besitzt in $T$ gleichmaissig den eru'eiterte'n absoluten Differentialquotientern.

Nach Annahme einer beliebig kleinen reellen positiven Grösse $\varepsilon$ lïsst sich nach der Voraussetzung für alle Werte $;$ in $T$ gleichzeitig eine hinreichend kleine reelle po 
sitive Grösse $\delta(\varepsilon)$ so bestimmen, dass, wenn

ist,

$$
|b|<\delta(\varepsilon)
$$

$$
|| \frac{f(z+b)-f(z)}{b}|-m(z)|<\varepsilon
$$

wird. Es soll zunächst gezeigt werden, dass $m(z)$ eine stetige Funktion von $z$ ist. Man bestimme einen festen Wert $h$, der der Bedingung genüge

Es wird

$$
|b|<\delta\left(\frac{\varepsilon}{3}\right) \text {. }
$$

$$
\begin{aligned}
& \left|m\left(z_{1}\right)-m(z)\right|=\mid\left(m\left(z_{1}\right)-\left|\frac{f\left(z_{1}+b\right)-f\left(z_{1}\right)}{b}\right|\right) \\
& +\left(\left|\frac{f\left(z_{1}+b\right)-f\left(z_{1}\right)}{b}\right|-\left|\frac{f(z+b)-f(z)}{b}\right|\right) \\
& +\left(\left|\frac{f(z+b)}{b}-f(z)\right|-m(z)\right) \mid \\
& \leq|| \frac{f\left(z_{1}+b\right)-f\left(z_{1}\right)}{b}|-m(z)|+\left|\frac{\left|f\left(z_{1}+b\right)-f\left(z_{1}\right)\right|-|f(z+b)-f(z)|}{b}\right| \\
& +\left|\frac{f(z+b)-f(z)}{b}\right|-m(z) \mid \\
& \leq \frac{\varepsilon}{3}+\left|\frac{\left(f\left(z_{1}+b\right)-f\left(z_{1}\right)\right)-(f(z+b)-f(z))}{h}\right|+\frac{\varepsilon}{3} \\
& =\frac{2 \varepsilon}{3}+\left|\frac{\left(f\left(z_{1}+b\right)-f(z+b)\right)-\left(f\left(z_{1}\right)-f(z)\right)}{b}\right| \\
& \leq \frac{2 \varepsilon}{3}+\frac{\left|f\left(\tilde{z}_{1}+b\right)-f(z+b)\right|+\left|f\left(z_{1}\right)-f(z)\right|}{|b|} \text {. }
\end{aligned}
$$

Wegen der Existenz des absoluten Differentialquotienten ist $f(\tilde{z})$ überall in $T$ stetig, also nach einem bekannten Satze gleichmässig sterig. Nach Annahme einer beliebig kleinen reellen positiven Grösse $\varepsilon$ ist also $\delta^{\prime}(\varepsilon)$ reell und positiv so bestimmbar, dass, wenn ein Wertepaar $z_{1}, z$ der Bedingung

genügt,

$$
\left|z_{1}-\tilde{z}\right|<\delta^{\prime}(\varepsilon)
$$

wird. Man bestimme

$$
\left|f\left(z_{1}\right)-f(z)\right|<\varepsilon
$$

$$
\left|z_{1}-z\right|<\delta^{\prime}\left(\frac{|b| \varepsilon}{6}\right) \text {, }
$$

dann wird

$$
\left|m\left(\tilde{n}_{1}\right)-m(z)\right|<\frac{2 \varepsilon}{3}+\frac{\varepsilon}{6}+\frac{\varepsilon}{6}=\varepsilon .
$$

Damit ist die Stetigkeit von $m(z)$ nachgewiesen.

Um die Existenz des erweiterten absoluten Differentialquotienten nachzuweisen, 
bestimme man $\delta^{\prime \prime}\left(\frac{\varepsilon}{2}\right)$ so klein, dass für alle $z_{1}$, für die

$$
\left|z_{1}-z\right|<\lambda^{\prime \prime}\left(\frac{\varepsilon}{2}\right)
$$

ist,

$$
\left|m\left(z_{1}\right)-m(z)\right|<\frac{\varepsilon}{2}
$$

wird. Ausserdem bestimme man

$$
\delta^{\prime \prime}\left(\frac{\varepsilon}{2}\right) \leq \frac{\mathrm{I}}{2} \delta\left(\frac{\varepsilon}{2}\right)
$$

Wenn dann auch

$$
\left|i_{2}-i_{1}\right|<\delta^{\prime \prime}\left(\frac{\varepsilon}{2}\right)
$$

so ist

also wird

$$
\left|z_{2}-z_{1}\right| \leq\left|z_{2}-\tilde{z}\right|+\mid z_{1}-\tilde{i}<2 \delta^{\prime \prime}\left(\frac{\varepsilon}{2}\right) \leq \delta\left(\frac{\varepsilon}{2}\right),
$$

$$
|| \frac{f\left(z_{2}\right)-f\left(\tilde{z}_{1}\right)}{z_{2}-i_{1}}\left|-m\left(\tilde{z}_{1}\right)\right|<\frac{\varepsilon}{2} .
$$

Man erhält unter Benutzung von (180) und (182)

$$
\begin{aligned}
& || \frac{f\left(\tilde{z}_{2}\right)-f\left(\tilde{i}_{1}\right)}{\tilde{i}_{2}-z_{1}}|-m(\tilde{z})|=\left|\left(\left|\frac{f\left(\tilde{i}_{2}\right)-f}{\tilde{i}_{2}-\tilde{z}_{1}}\right|-m\left(\tilde{z}_{1}\right)\right)+\left(m\left(\tilde{z}_{1}\right)-m(z)\right)\right| \\
& \leq|| \frac{f\left(z_{2}\right)-f\left(z_{1}\right)}{z_{2}-z_{1}}\left|-m\left(z_{1}\right)\right|+\left|m\left(\tilde{z}_{1}\right)-m(z)\right|<\frac{\varepsilon}{2}+\frac{\varepsilon}{2}=\varepsilon,
\end{aligned}
$$

also existiert an jedem Punkie $\approx$ der erweiterte absolute Differentialquotient und zwar gleichmässig, weil ( 179 ) und ( $18 \mathrm{I}$ ) gleichmässig für alle $z$ in $T$ erfüllbar sind. Die Voraussetzungen des Satzes (II) sind also gegeben.

Der Satz (II) lüsst sich folgendermassen formulieren: Besit $t$ eine eindeutige Funktion $f(\tilde{i})$ einer kumplexen Variablen $\tilde{\imath}$ in einem abgesiblossenen, einjach zllammenbangenden Gitiet' GLEICHмässig den geiü̈bnlicben absoluten Differentialquotienten, so ist enti'e'der $f(\tilde{z})$ wider ibre kunjugie'rte $\overline{f(}(\tilde{)})$ eine analytiscbe Funktion.

Den analogen Satz für den erweiterten Signum-Differentialquotienten kann ich nur unter einschränkenden Voraussetzungen beweisen, die gewisse Anforderungen stellen an die Schnelligkeit der Annäherung des Signums des erweiterten Differenzenquotienten an den Signum-Differentialquotienten.

Satz III. - Es sii die reelle positize Konstante $k_{0}>$ I definierl als die Läsung der transzendenten Gleichung

ferner $K_{\mathrm{u}}$ durib die Gleichung

$$
\log k+\frac{\mathrm{I}}{k^{2}}=\mathrm{I}
$$

$$
K_{\mathrm{o}}=\frac{21 \overline{k_{\mathrm{o}}^{2}}-\mathrm{I}}{\log k_{\mathrm{o}}}=\frac{2 k_{\mathrm{o}}^{2}}{\sqrt{k_{\mathrm{o}}^{2}-\mathrm{I}}} .
$$

Die cindtutige Funktion $f(\tilde{z})$ der komplexen l'ariablen z genüge in dem einfach zusam- 
menbängenden, abgescblossenen Gebiete $T$ folgender Bedingung. Fir jeden eiñelnen VVert von $z$ in $T$ existiere fur alle reellen positiven

$$
\delta<\delta_{0}(z)
$$

wo $\delta_{0}(\tilde{\tau})$ eine nur l'on $z$ abbaingige Konstante, eine reelle positive Funktion won $\log \delta^{-1}$

$$
\tau\left(\chi, \log \delta^{-1}\right)<\frac{\mathrm{I}}{K_{\mathrm{o}}},
$$

die mit wachsendem $\log \delta^{-1},(d . b$. mit abnebmendem $\delta)$ nie zunimmt, und der Bedingung genügt,

$$
\int_{\log \delta_{0}^{-1}}^{\infty} \frac{\tau\left(z, \log \delta^{-1}\right)}{\log \delta^{-1}} d\left(\log \delta^{-1}\right)=\infty
$$

derart, dass, w'enn

stets

$$
\left|z_{1}-z\right| \text { und }\left|\tilde{z}_{2}-z\right|<\delta
$$

$$
\left|\operatorname{sgn} \frac{f\left(z_{2}\right)-f\left(z_{1}\right)}{i_{2}-z_{1}}-s(z)\right|<\frac{\frac{1}{K_{0}}-\tau\left(\tilde{z}, \log \delta^{-1}\right)}{\log \delta^{-1}}
$$

ist, wo $|s(z)|=\mathrm{I}$. Dann ist $f(z)$ eine reguläre analytisibr Funktion zon $z$, deren Ableitung in $T$ von o verschieden ist.

Es soll wieder bewiesen werden, dass das Integral über $f(i)$, erstreckt uber den Umfang eines beliebigen Dreiecks, gleich o ist, woraus dasselbe für das Integral uber einen beliebigen geschlossenen, aus geraden Stucken bestehenden Weg folgt. Hieraus folgt wieder nach dem verallgemeinerten Morera'schen Satze, dass $f(i)$ eine in $T$ reguläre analytische Funktion sein muss. Un aber den Morera'schen Satz anwenden zu können, muss bewiesen werden, dass $f(\tau)$ eine stetige Funktion ist.

Hilfssatz IV. - Besitat eine Funktion $f(\tilde{\imath})$ an einer Stelle $z=z_{0}$ einen eru'eiterten Signum.Differentialquotienten, so ist sie an dieser Stelle stetig.

Nach Annahme einer beliebig kleinen reellen positiven Grösse $\delta$ bezeichne $n(\delta)$ die obere Grenze aller Werte, die

$$
\left|\operatorname{sgn} \frac{f\left(z_{2}\right)-f\left(z_{1}\right)}{z_{2}-z_{1}}-s\left(z_{0}\right)\right|
$$

annimmt, wenn $z_{1}$ und $z_{2}$ alle Wertepaare ungleicher Zahlen durchlaufen, die der Bedingung

$$
\left|z_{1}-z_{0}\right| \leq \delta, \quad\left|z_{2}-z_{0}\right| \leq \delta
$$

genügen. Dann bedeutet die Existenz des erweiterten Signum-Differentialquotienten

Man setze

$$
\lim _{\delta=0} n(\delta)=0 \text {. }
$$

$$
\begin{aligned}
& \varepsilon_{0}=\left|z_{1}-z_{2}\right|, \quad e_{1}=\left|z_{2}-z_{0}\right|, \quad e_{2}=\left|z_{0}-z_{1}\right|, \\
& E_{0}=\left|f\left(z_{1}\right)-f\left(z_{2}\right)\right|, \quad E_{1}=\left|f\left(z_{2}\right)-f\left(z_{0}\right)\right|, \quad E_{2}=\left|f\left(z_{0}\right)-f\left(z_{1}\right)\right|,
\end{aligned}
$$


ferner

( 187 )

$$
x_{0}=\frac{z_{2}=z_{0}}{z_{1}-z_{0}}, \quad x_{1}=\frac{z_{0}-z_{1}}{z_{2}-z_{1}}, \quad x_{2}=\frac{z_{1}-z_{2}}{z_{0}-z_{2}},
$$

$$
X_{0}=\frac{f\left(z_{2}\right)-f\left(z_{0}\right)}{f\left(z_{1}\right)-f\left(\tilde{z}_{0}\right)}, \quad X_{1}=\frac{f\left(z_{0}\right)-f\left(z_{1}\right)}{f\left(z_{2}\right)-f\left(z_{1}\right)}, \quad X_{2}=\frac{f\left(z_{1}\right)-f\left(z_{2}\right)}{f\left(z_{0}\right)-f\left(z_{2}\right)},
$$

Es ist

$$
q_{0}=e_{1}^{2}+e_{2}^{2}-e_{0}^{2}, \quad q_{1}=e_{2}^{2}+e_{0}^{2}-e_{1}^{2}, \quad q_{2}=e_{0}^{2}+e_{1}^{2}-e_{2}^{2} \text {. }
$$

$$
x_{1}=\frac{1}{1-x_{0}}, \quad x_{2}=\frac{1}{1-x_{1}}, \quad x_{0}=\frac{1}{1-x_{2}},
$$

also haben die Imaginärteile von $x_{0}, x_{1}, x_{2}$ das gleiche Vorzeichen $\omega$. Es ist also nach (I59) und (I60)

ferner nach (158)

$$
x_{n}=\frac{q_{0}+\omega i \imath^{\prime} \overline{D\left(e_{1}, \overline{e_{2}, e_{0}}\right)}}{2 e_{2}^{2}},
$$

Also wird

$$
\left|x_{0}\right|=\frac{e_{1}}{e_{2}},
$$

$$
\operatorname{sgn} x_{0}=\frac{q_{0}+\omega i 1^{\prime} \overline{D\left(e_{1}, e_{2}\right.}, \overline{\left.e_{0}\right)}}{2 e_{1} e_{2}},
$$

ebenso

$$
\operatorname{sgn} x_{1}=\frac{q_{1} \pm \omega i \eta^{\prime} \overline{D\left(e_{1}, e_{2}\right.}, \overline{\left.e_{0}\right)}}{2 e_{2} e_{0}},
$$

Es ist :llso

(193)

$$
\operatorname{sgn} x_{2}=\frac{q_{2}+\omega i 1 / \overline{D\left(e_{1}, e_{2},\right.} \underline{\left.e_{0}\right)}}{2 e_{0} e_{1}} \text {. }
$$

Es werde gesetzt

$$
e_{0}: e_{1}: e_{2}=3\left(\operatorname{sgn} x_{0}\right): \Im\left(\operatorname{sgn} x_{1}\right): 3\left(\operatorname{sgn} x_{2}\right) \text {. }
$$

$$
\left\{\begin{array}{l}
\operatorname{sgn}\left(f\left(z_{2}\right)-f\left(z_{1}\right)\right)=s\left(z_{0}\right) \cdot \operatorname{sgn}\left(z_{2}-z_{1}\right) \cdot\left(1+x_{0}\right), \\
\operatorname{sgn}\left(f\left(z_{0}\right)-f\left(z_{2}\right)\right)=s\left(z_{0}\right) \cdot \operatorname{sgn}\left(z_{0}-z_{2}\right) \cdot\left(1+x_{1}\right), \\
\operatorname{sgn}\left(f\left(z_{1}\right)-f\left(z_{0}\right)\right)=s\left(z_{0}\right) \cdot \operatorname{sgn}\left(z_{1}-z_{0}\right) \cdot\left(1+z_{2}\right) .
\end{array}\right.
$$

Hierin ist nach (186)

ebenso

$$
\left|s\left(z_{0}\right) \cdot x_{0}\right|=\left|x_{0}\right| \leqslant n(\delta),
$$

Ferner ist

oder

oder

$$
\left|x_{1}\right| \leq n(\delta), \quad\left|x_{2}\right| \leq n(\delta) .
$$

$$
\left|\mathrm{I}+x_{\mathrm{o}}\right|=\mathrm{I}
$$

$$
I+x_{0}+\bar{x}_{0}+x_{0} \bar{x}_{0}=I
$$

also

$$
\Re\left(x_{0}\right)=-\frac{\left|x_{0}\right|^{2}}{2},
$$

$$
\Im\left(x_{0}\right)= \pm \sqrt{\left|x_{0}\right|^{2}-\frac{\left|x_{0}\right|^{4}}{4}}= \pm\left|x_{0}\right| \cdot \sqrt{1-\frac{\left|x_{0}\right|^{2}}{4}},
$$


ebenso für $\%_{1}$ und $x_{2}$. Setzt man (194) in (188) ein, so erhält man

(195) $\operatorname{sgn} X_{0}=\operatorname{sgn} x_{0} \cdot \frac{1+x_{1}}{1+x_{2}}=\operatorname{sgn} x_{0} \cdot\left(1+x_{1}\right)\left(1+\bar{x}_{2}\right) \equiv \operatorname{sgn} x_{0} \cdot\left(1+\lambda_{0}\right)$, ebenso

(196)

$$
\begin{aligned}
& \operatorname{sgn} X_{1} \equiv \operatorname{sgn} x_{1} \cdot\left(1+\lambda_{1}\right), \\
& \operatorname{sgn} X_{2} \equiv \operatorname{sgn} x_{2} \cdot\left(1+\lambda_{2}\right),
\end{aligned}
$$

(197)

hierin sind $\lambda_{1}, \dot{\lambda}_{2}, \lambda_{3}$ zur Abkürzung eingeführt. Es ist ebenso

$$
\left|\lambda_{0}\right|=\left|x_{1}+\left(1+x_{1}\right) \bar{x}_{2}\right| \leq\left|x_{1}\right|+\left|\left(1+x_{1}\right) \bar{x}_{2}\right|=\left|x_{1}\right|+\left|x_{2}\right| \leqslant 2 n(\delta),
$$

$$
\begin{aligned}
& \left|\lambda_{1}\right| \leq 2 n(\delta), \\
& \left|\lambda_{2}\right| \leq 2 n(\delta) .
\end{aligned}
$$

Aus den zu (189) und (193) analogen Gleichungen, die sich auf die $X_{0}, E_{0}$ usw. beziehen, unter Benutzung von (195), (196), (I97), (191), (192) und schliesslich der (I59) analogen Gleichung für $\mathfrak{R}\left(x_{\mathrm{o}}\right)$ erhält man

$$
\begin{aligned}
& \int \frac{f\left(z_{2}\right)-f\left(\tilde{z}_{0}\right)}{f\left(z_{1}\right)-f\left(z_{0}\right)}=X_{0}=\operatorname{sgn} X_{0} \cdot \frac{E_{1}}{E_{2}}=\operatorname{sgn} X_{0} \cdot \frac{\mathfrak{s}\left(\operatorname{sgn} X_{1}\right)}{\mathfrak{I}\left(\operatorname{sgn} X_{2}\right)}
\end{aligned}
$$

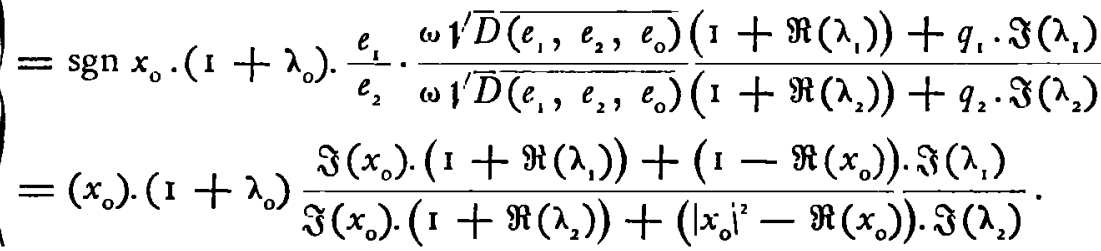

Setzt man

(199)

$$
X_{0}=x_{0}+\xi_{0}
$$

so ist

(200)

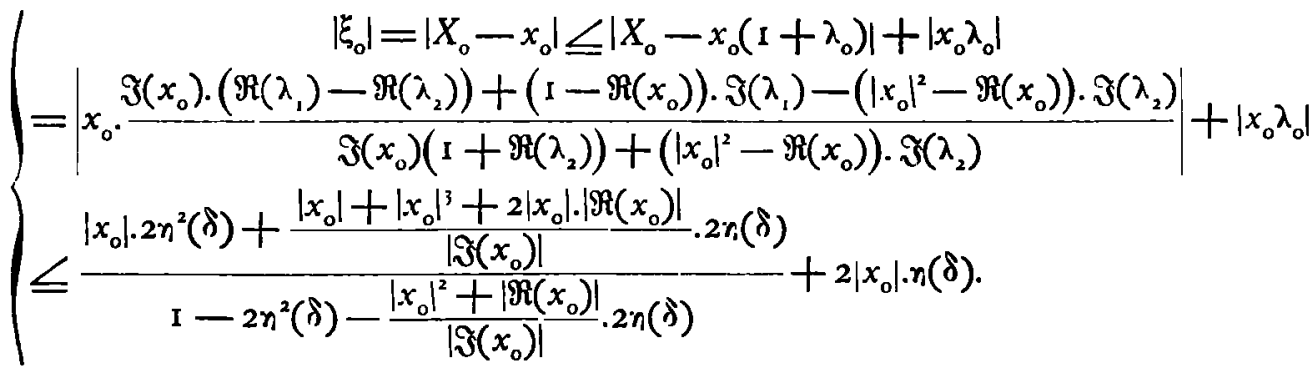

Man denke sich $r_{1}(\delta)$ hierin so klein bestimmt, dass der Nenner nicht verschwindet und

(201)

$$
n(\delta)<\frac{1}{100}
$$

Man betrachte diejenigen $x_{0}$, die der Bedingung genügen

(202) und unterscheide zwei Fälle.

$$
\left|x_{0}\right| \leq \mathrm{I}
$$

Fall (a): Es sei

$$
\left|\Re\left(x_{0}\right)\right| \leq\left|\Im\left(x_{0}\right)\right|
$$


Es wird nach (200)

$\left(20_{4}\right)$

$$
\left\{\begin{aligned}
\left|\xi_{0}\right| & \leq n(\delta) \cdot\left(\frac{2 n(\delta)+4+4 \sqrt{2}}{1-2 n^{2}(\delta)-(2+2 \sqrt{2}) n(\delta)}+2\right) \\
& <n(\delta) \cdot\left(\frac{2 n(\delta)+10}{1-2 n^{2}(\delta)-5 n(\delta)}+2\right) \\
& <n(\delta) \cdot\left(\frac{100200}{9498}+2\right)<\operatorname{I~} 3 n(\delta) .
\end{aligned}\right.
$$

(205)

Fall $(b)$ : Es sei

Man setze

$$
\left|\Re\left(x_{0}\right)\right| \supseteq\left|\mathfrak{F}\left(x_{0}\right)\right|
$$

$$
x_{\mathrm{o}}=i x_{\mathrm{o}}^{\prime \prime}, \quad i=x_{\mathrm{o}}^{\prime},
$$

ferner sei $z^{\prime}$ definiert durch die Gleichung

Man setze ferner

$$
\frac{z^{\prime}-z_{0}}{z_{1}-z_{0}}=i
$$

Ferner sei

$$
\begin{aligned}
& \frac{f\left(z^{\prime}\right)-f\left(z_{0}\right)}{f\left(z_{1}\right)-f\left(z_{0}\right)}=X_{0}^{\prime}, \\
& \frac{f\left(z_{2}\right)-f\left(z_{0}\right)}{f\left(i^{\prime}\right)-f\left(z_{0}\right)}=X_{0}^{\prime \prime} .
\end{aligned}
$$

Es ergibt sich

$$
\begin{aligned}
& X_{0}^{\prime}=x_{0}^{\prime}+\xi_{0}^{\prime}=i+\xi_{0}^{\prime}, \\
& X_{0}^{\prime \prime}=x_{0}^{\prime \prime}+\xi_{0}^{\prime \prime} .
\end{aligned}
$$

$$
X_{0}=X_{0}^{\prime} \cdot X_{0}^{\prime \prime}=\left(i+\xi_{0}^{\prime}\right)\left(x_{0}^{\prime \prime}+\xi_{0}^{\prime \prime}\right)=i x_{0}^{\prime \prime}+i \xi_{0}^{\prime \prime}+x_{0}^{\prime \prime} \xi_{0}^{\prime}+\xi_{0}^{\prime} \xi_{0}^{\prime \prime}=x_{0}+\xi_{0},
$$

also wird

(206)

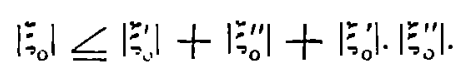

Es genügt nach (200) $\zeta_{0}^{\prime}$ der Bedingung

$$
\left|\xi_{0}^{\prime}\right| \leq n(\delta) \cdot\left(\frac{2 n(\delta)++}{1-2 n^{2}(\delta)-2 n(\delta)}+2\right)<n(\delta)\left(\frac{40200}{9798}+2\right)<7 n(\delta) .
$$

Da $x_{0}$ der Bedingung (205) genügt, so genügt $x_{0}^{\prime \prime}$ der Bedingung (203), also $\xi_{0}^{\prime \prime}$ der Bedingung (204). Also wird nach (206)

$$
\text { (207) } \quad\left|\xi_{0}\right|<n(\delta) \cdot(7+13+7 \cdot 13 \cdot n(\delta))<2 \operatorname{1n}(\delta) \text {. }
$$

(207) gilt also für alle $\xi_{0}$, deren $x_{0}$ (202) genügt.

Es sei $x_{0}$ von nun an ein fester nicht reeller Wert, dessen absoluter Betrag kleiner sei als r. Für die Zwecke des zu beweisenden Hilfssatzes könnte man $x_{0}$ im übrigen willkürlich wählen. Ich will aber gleich denjenigen Wert von $x_{0}$ ermitteln, der für $\left|X_{\mathrm{o}}\right|$ die günstigste Abschätzung liefert, da ich diesen zum Beweise des Hauptsatzes (III) 
brauche. Aus (198) folgt

(208) $\left|X_{0}\right| \leq\left|x_{1}\right| \cdot\left(1+\frac{2 n^{2}(\delta)+\frac{1-\Re\left(x_{0}\right)+\left.|| x_{0}\right|^{2}-\Re\left(x_{0}\right) \mid}{\left|\Im\left(x_{0}\right)\right|} \cdot 2 n(\delta)}{1-2 n^{2} \delta-\frac{\left.|| x_{0}\right|^{2}-\Re\left(x_{0}\right) \mid}{\left|\Re\left(x_{0}\right)\right|} .2 n(\delta)}\right)$.

Denkt man sich die Klammer nach Potenzen von $n(\delta)$ entwickelt, so lautet der Koeffizient der ersten Potenz von $2 n(\delta)$ in der Klammer

$$
\frac{\mathrm{I}-\mathfrak{R}\left(x_{\mathrm{o}}\right)+\left.|| x_{\mathrm{o}}\right|^{2}-\Re\left(x_{\mathrm{o}}\right) \mid}{\left|\mathfrak{\Im}\left(x_{\mathrm{o}}\right)\right|}=C .
$$

Man halte zunächst $\left|x_{\mathrm{o}}\right|$ fest und verfüge über $\operatorname{sgn} x_{\mathrm{o}}$ so, dass dieser Koeffizient möglichst klein werde. Es sind zwei Fälle zu unterscheiden.

Fall $(a)$ : Es sei

Dann ist

$$
\Re\left(x_{0}\right) \gg\left|x_{0}\right|^{2}
$$

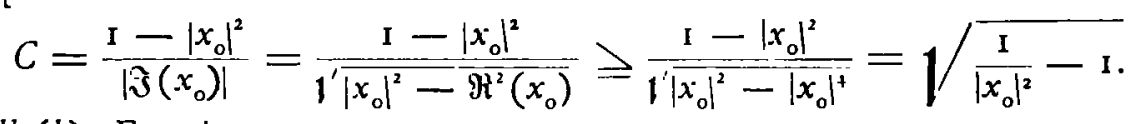

(209)

Fall (b): Es sei

Dann ist

$$
\Re\left(x_{0}\right) \leq\left|x_{0}\right|^{2}
$$

(2I0)

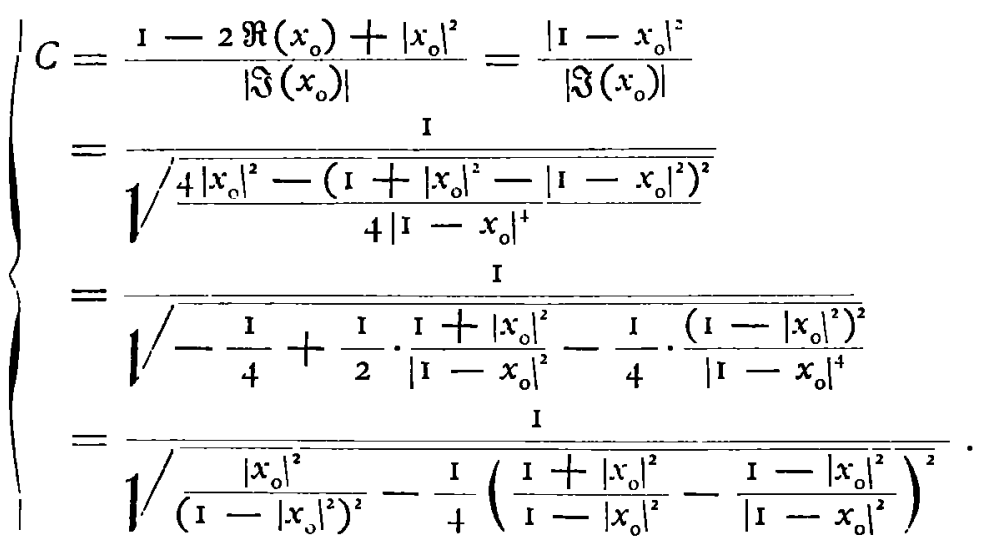

Da nach (209)

so folgt aus (210)

$$
\left|1-x_{0}\right|^{2}=1-2 \Re\left(x_{0}\right)+\left|x_{0}\right|^{2} \supseteq I-\left|x_{0}\right|^{2},
$$

$$
C \geqslant \frac{\mathrm{I}}{\sqrt{\frac{\left|x_{0}\right|^{2}-\left|x_{0}\right|^{2}}{\left(1-\left|x_{0}\right|^{2}\right)^{2}}}}=1 \sqrt{\frac{\mathrm{I}}{\left|x_{0}\right|^{2}}-\mathrm{I}}
$$

Inı Falle (a) wie in Falle (b) wird das Minimum von $C$ erreicht für

also

$$
\Re\left(x_{0}\right)=\left|x_{0}\right|^{2}
$$

$$
\begin{gathered}
x_{\mathrm{o}}=\left|x_{\mathrm{o}}\right|\left(\left|x_{\mathrm{o}}\right|+i 1^{\prime} \overline{\mathrm{I}-\left|x_{\mathrm{o}}\right|^{2}}\right), \\
C=\sqrt{\frac{\mathrm{I}}{\left|x_{\mathrm{o}}\right|^{2}}-\mathrm{I}}
\end{gathered}
$$


(208) lautet in diesem speziellen Falle

(2I2)

$$
\left\{\begin{aligned}
\left|X_{0}\right| & \leq\left|x_{0}\right| \cdot\left(\mathrm{I}+\frac{2 C \cdot n(\delta)+2 n^{2}(\delta)}{1-2 n^{2}(\delta)}\right) \\
& =\left|x_{0}\right| \cdot\left(\mathrm{I}+2 C \cdot n(\delta)+2 n^{2}(\delta) \cdot \frac{2 C \cdot n(\delta)+1}{1-2 n^{2}(\delta)}\right) \\
& <\left|x_{0}\right| \cdot\left(1+2 C \cdot n(\delta)+n^{2}(\delta) \frac{10000}{4999}\left(\frac{C}{50}+1\right)\right) .
\end{aligned}\right.
$$

Es sei nun $z$ eine Zahl, die der Bedingung

$$
\left|z-z_{0}\right| \leq\left|z_{1}-z_{0}\right|
$$

genüge. Man definiere eine Reihe von Zahlen

durch die Gleichung

$$
z_{1}, z_{2}, z_{3}, \ldots, z_{2}, \ldots
$$

Ferner sei $n$ so definiert, dass

$$
z_{v}-z_{0}=\left(z_{1}-z_{0}\right) \cdot x_{0}^{\nu-1} \text {. }
$$

$$
\left|z_{n}-z_{0}\right| \geqslant\left|z-z_{0}\right|>\left|z_{n+1}-z_{0}\right| .
$$

Dann ist nach (207) und (212)

(213)

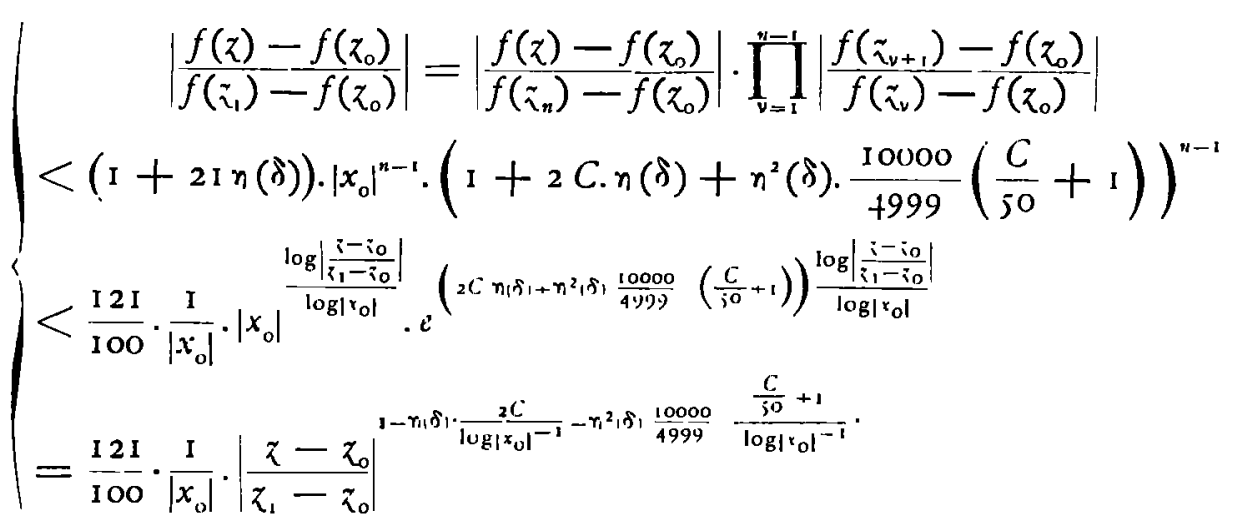

Daraus folgt aber, dass $\left|f(z)-f\left(z_{3}\right)\right|$ mit abnehmendem $\left|z-z_{0}\right|$ beliebig klein wird, also ist $f(z)$ an der Stelle $z=z_{0}$ stetig. Es ist aber noch mehr bewiesen worden.

Nach Anuahme einer beliebig kleinen reellen positiven Grösse $\varepsilon$ lässt sich eine hinreichend grosse reelle positive Zahl $\lambda$ und eine hinreichend kleine reelle positive Zahl $\delta$ so bestinmen, dass für alle $\tilde{\tau}$, die der Bedingung

genügen,

$$
\left|z-z_{\mathrm{o}}\right|<\delta
$$

$$
\left|f(z)-f\left(z_{0}\right)\right|<\lambda_{0}\left|z-z_{0}\right|^{i-\frac{\varepsilon}{2}}
$$

wird. Bestimmt man ferner $\delta^{\prime} \leq \delta$ so klein, dass

$$
\lambda<\delta^{-\frac{\varepsilon}{2}} \text {, }
$$


so wird, wenn

ist,

$$
\begin{gathered}
\left|z-i_{0}\right|<\delta^{\prime} \\
|f(z)-f(\tilde{z})|<\left|z-z_{0}\right|^{\prime-\varepsilon} .
\end{gathered}
$$

Damit rechtfertigt sich auch die IVahl des Beispiels $(F)$

$$
f(\tilde{i})=\tilde{z} \cdot \log |\tilde{z}|^{-i},
$$

in dem also der Logarithmus durch keine noch so kleine Potenz von $|z|^{-1}$ hätte ersetzt werden dürfen.

[Aehnliche Eiuschränkungen wüden sich unter der Voraussetzung der Existenz des erweiterten ahsulute'n Differentialquotienten an einer Stelle $z=\tilde{z}_{0}$ für $\operatorname{sgn}\left(f(z)-f\left(\tilde{z}_{0}\right)\right.$ ) finden lassen; doch gehe ich daraut nicht ein. Zum Beweise des Satzes (II) waren sie ja nicht nötig].

Bevor ich z.um Beve eise des Satzes (III) übergehe, will ich für den Koeffizienten ron $r(\hat{)})$ im Exponenten des letzten Ausdiucks in (2I3) das Minimum bestimmen. Iich setze

$$
\left|x_{0}\right|^{-1}=k \text {. }
$$

Es handelt sich also um das Minimum $K_{0}$, ron

$$
\frac{2 C}{\log \left|x_{0}\right|^{-1}}=\frac{2 \sqrt{k^{2}-1}}{\log k}=K
$$

für $k>$ I. Ditser Ausdruck wird mit Annäherung von $k$ an I unendlich gross, wird ferner unendlicb gross fur unendlich grosse Werte von k. Dazwischen muss mindestens ein Minimum liegen. Es ist

$$
\mathrm{o}=\frac{d}{d k}\left(\frac{1 \overline{k^{2}-1}}{\log k}\right)=\left(\frac{k \log k}{\mathfrak{l}^{\prime} \overline{k^{2}-\mathrm{I}}}-\frac{1 \overline{k^{2}-\mathrm{I}}}{k}\right) \cdot \frac{\mathrm{I}}{\log ^{2} k}=\frac{k^{2} \log k-k^{2}+\mathrm{I}}{k \cdot 1 \overline{k^{2}-\mathrm{I}} \cdot \log ^{2} k}
$$

wenn

$$
\log k+\frac{\mathrm{I}}{k^{2}}=\mathrm{I}
$$

Diese Gleichung ist zunächst erfullt für den nicht brauchbaren Wert $k=1$. Die linke Seite von (2I 4 ) wird positiv unendlich gross für $k=0$ und für $k=\infty$. Dazwischen muss mindestens ein Minimun liegen. Es ist

$$
\mathrm{o}=\frac{d}{d k}\left(\log k+\frac{\mathrm{I}}{k^{2}}\right)=\frac{\mathrm{I}}{k}-\frac{2}{k^{3}}
$$

fiur $k= \pm 1$ ' Fur $k>1$ kommt nur der Wert $k=\sqrt{2}$ in Betracht, existiert also auch nur ein Minimum der linken Seite von $\left(2 r_{+}\right)$und kein Maximum.

Für $k=1 / 2$ wird

$$
\log k+\frac{1}{k^{2}}=\frac{1}{2} \log 2+\frac{1}{2}<\mathrm{I} .
$$

Also nimme die linke Seite ron ( $\mathrm{I}_{4}$ ) den Wert I genau noch einmal an für einen 
Wert $k_{\mathrm{o}}>1^{/ 2}$; und $\mathrm{zwar}$ liegt dieser Wert im Intervall

$$
2,21<k_{\mathrm{o}}<2,22 .
$$

Also kann auch $K$ nur an dieser Stelle sein Minimum haben. Es ist ferner wegen (2 I 4 )

also liegt $K_{\mathrm{o}}$ im Intervall

$$
K_{\mathrm{o}}=\frac{2 k_{\mathrm{n}}^{2}}{1^{\prime} \overline{k_{\mathrm{o}}^{2}-\mathrm{I}}}
$$

Es ist nach (2I I)

$$
\begin{gathered}
{ }^{4,93}<K_{\mathrm{o}}<4,98 . \\
C_{\mathrm{o}}=\mathrm{I}^{\prime} \overline{k_{\mathrm{o}}^{2}-\mathrm{I}}
\end{gathered}
$$

also liegt $C_{\mathrm{o}}$ im Intervall

$$
\text { I,97 }<C_{\mathrm{o}}<\mathrm{I}, 99
$$

Bezeichnet man mit $\int_{a_{a_{1}, r_{2}, n_{3}}}$ das geschlossene Integral üher die Strecken $a_{2} \ldots a_{3}$, $a_{;} \ldots a_{1}, a_{1} \ldots a_{2}$ so gilt die Identität

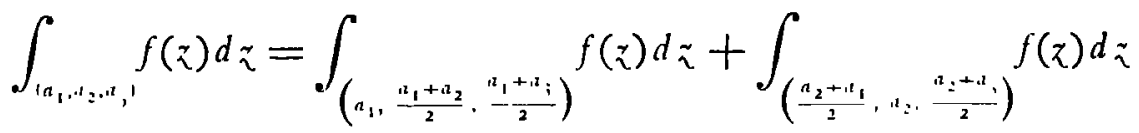

$$
\begin{aligned}
& +\int_{\left(\frac{a_{3}+a_{1}}{2}, \frac{a_{j}+a_{2}}{2}, a_{3}\right)} f(\tilde{z}) d z+\int_{\left(\frac{a_{z}+x_{j}}{2}, \frac{a_{j}+a_{1}}{2} \cdot \frac{a_{1}+a_{2}}{2}\right)} f(z) d \tilde{\imath} .
\end{aligned}
$$

Wenn die linke Seite dieser Gleichung von o versihieden, so können auch nicht alle vier rechtsstehenden Integrale o sein. Man bezeichne die Ecken des Integrationsweges desjenigen Integrals der rechten Seite, das den grössten absoluten Betrag besitzt, der Reihe nach mit $a_{1}^{\prime}, a_{2}^{\prime}, a_{;}^{\prime}$. Sollten die absoluten Betrage mehrerer Integrale der rechten Seite einander gleich sein, so bevorzuge man das in der Summe voranstehende Inte. gral. Man erhält

$$
\left|\int_{\left|a_{1}, a_{2}, u_{3}\right|} f(i) d z_{1} \leq 4\right| \int_{\left.\mid a_{1}^{\prime}, a_{2}^{\prime}, i_{3}^{\prime}\right\}} f(z) d z \mid .
$$

Auf das Integral der rechten Seite wende man wiederholt dieselbe Formel an und erhalt

$$
\left|\int_{\left.\mid a_{1}, a_{2}, a_{3}\right)} f(z) d z\right| \leq 4^{r} \cdot\left|\int_{\left(a_{1}^{\prime r}, i_{2}^{\prime r)}, a_{3}^{(r)}\right.} f(\tilde{z}) d z\right|
$$

wo $r$ eine positive ganze Zahl. Denkt man sich $a_{1}^{(r)}, a_{2}^{|r|}, a_{3}^{(r)}$ für beliebig grosse $r$ ermittelt, so wird

$$
\lim _{r=\infty} a_{1}^{(r)}=\lim _{r=\infty} a_{2}^{r \prime}=\lim _{r=\infty} a_{;}^{(r)}=i_{0}
$$

Man bestimme nun zunä:hst eine reelle positive ganze Zahl $n_{\mathrm{v}}$ erstens so gross dass

$$
\frac{\mathrm{I}}{k_{\mathrm{o}}^{n_{0}}}<\hat{\delta}_{0}\left(\tilde{i}_{0}\right)
$$


zweitens so gross, dass

(217)

$$
\frac{1}{K_{\mathrm{o}} n_{\mathrm{o}} \cdot \log k_{\mathrm{o}}} \leq \frac{\mathrm{I}}{\mathrm{IO0}}
$$

oder dass

$$
n_{\mathrm{o}} \supseteq \frac{50}{1^{\prime} \frac{50}{k_{0}^{2}}-\mathrm{I}}=\frac{50}{C_{0}}
$$

Hierzu genügt es nach (215),

$$
n_{\mathrm{o}} \supseteq 26
$$

zu machen. Sodann bestimme man einen IVert $\tilde{i}_{1}$, derart dass

$$
\left|z_{1}-z_{0}\right|=k_{0}^{-n_{0}} \text {. }
$$

Dann wird nach (185) und (217)

also ist (20I) erfüllt.

$$
n\left(\left|z_{1}-z_{0}\right|\right)<\frac{1}{K_{0} \cdot n_{0} \cdot \log k_{0}} \leq \frac{1}{100}
$$

Wegen $(184)$ divergiert

$$
\sum_{v=1}^{\infty} \frac{\tau\left(z_{0},\left(n_{0}+v-\mathrm{I}\right) \log k_{\mathrm{c}}\right)}{\left(n_{0}+v-\mathrm{I}\right) \log k_{\mathrm{o}}} \geqslant \frac{\mathrm{I}}{\log k_{\mathrm{o}}} \cdot \int_{n_{0} \log k_{0}}^{\infty} \frac{\tau\left(z_{0}, \log \delta^{-1}\right)}{\log \delta^{-1}} d\left(\log \delta^{-1}\right)=\infty .
$$

Also ist

$$
\prod_{\nu=1}^{\infty}\left(\mathrm{I}-\frac{\mathrm{I} S}{4} \cdot \frac{\tau\left(z_{0},\left(n_{0}+\nu-\mathrm{I}\right) \log k_{0}\right)}{\left(n_{0}+\nu-\mathrm{I}\right)}\right)=0 .
$$

Es ist wegen $(183)$ und $(217)$

$$
\mathrm{I}-\frac{\mathrm{I} S}{4} \cdot \frac{\tau\left(z_{0},\left(n_{\mathrm{o}}+\mathrm{v}-\mathrm{I}\right) \log k_{\mathrm{o}}\right)}{\left(n_{\mathrm{o}}+\mathrm{v}-\mathrm{I}\right) \log k_{\mathrm{o}}}>\mathrm{I}-\frac{\mathrm{I} S}{\mathrm{t}} \cdot \frac{\mathrm{I}}{n_{\mathrm{o}} K_{\mathrm{o}} \log k_{\mathrm{o}}}>\mathrm{I}-\frac{3}{8 \mathrm{o}},
$$

also sind alle Faktoren des unendlichen Produktes (219) positiv. Man bestimme nun $n$ so gross, dass

(220) $\prod_{v=1}^{n-1}\left(1-\frac{15}{4} \cdot \frac{-\left(z_{0},\left(n_{0}+v-1\right) \log k_{0}\right)}{\left(n_{0}+v-1\right) \log k_{0}}\right)<\frac{3 \varepsilon}{4 A_{1}^{2}} \cdot\left|\frac{z_{1}-z_{0}}{f\left(z_{1}\right)-f\left(z_{0}\right)}\right|$,

wo $A_{1}, A_{2}, A_{3}$ durch die Gleichungen (I4I) und (I42) definiert seien. Man setze wieder

worin

$$
z_{n}-z_{0}=\left(z_{1}-z_{0}\right) \cdot x_{0}^{n-1}
$$

$$
x_{0}=\frac{1}{k_{0}}\left(\frac{1}{k_{0}}+i \sqrt{\frac{\mathrm{I}}{k_{0}^{2}}-\mathrm{I}}\right) \text {. }
$$

Dann erhält man durch wiederholte Anwendung von (2I2) und unter Benutzung 
von $(585)$

$$
\left|\frac{f\left(z_{11}\right)-f\left(z_{0}\right)}{f\left(z_{1}\right)-f\left(z_{0}\right)}\right|
$$

$<k_{0}^{-\mid t-11} \prod_{y=1}^{n-1}\left(\mathrm{I}+2 C_{0} \cdot r_{1}\left(k_{0}^{-n_{0}-v+1}\right)+3 n^{2}\left(k_{0}^{-n_{0}-n+1}\right)\right)$

$<\left|\frac{z_{n}-z_{0}}{\tilde{z}_{1}-z_{0}}\right| \cdot \prod_{\nu=1}^{n-1}\left(\mathrm{I}+\frac{2 C_{0}}{K_{0}\left(n_{0}+v-\mathrm{I}\right) \log k_{0}}\right.$.

$$
\left.-\frac{2 C_{0} \cdot \tau\left(z_{0},\left(n_{0}+v-\mathrm{I}\right) \log k_{0}\right)}{\left(n_{0}+v-\mathrm{I}\right) \log k_{0}}+\frac{3}{K_{0}^{2}\left(n_{0}+v-\mathrm{I}\right)^{2} \log ^{2} k_{0}}\right)
$$

(22I)

$$
\begin{aligned}
& \left\{\frac{z_{n}-z_{0}}{\mid i_{1}-z_{0}} \mid \cdot \prod_{\nu=1}^{u-1} j\left(1+\frac{1}{n_{0}+v-I}\right)\left(\mathrm{I}+\frac{1}{5\left(n_{0}+n-\mathrm{I}\right)^{2}}\right)\right. \\
& \left.-\frac{2 C_{0}}{\left(\mathrm{I}+\frac{1}{26}\right)\left(\mathrm{I}+\frac{1}{3 ; 80}\right)} \cdot \frac{\tau\left(\tilde{i}_{0},\left(n_{0}+v-\mathrm{I}\right) \log k_{0}\right)}{\left(n_{0}+v-\mathrm{I}\right) \log k_{0}} \cdot\left(1+\frac{\mathrm{I}}{n_{0}+\nu-\mathrm{I}}\right)\left(\mathrm{I}+\frac{\mathrm{I}}{5\left(n_{0}+v-\mathrm{I}\right)^{2}}\right)\right\} \\
& <\left|\frac{\tilde{z}_{n}-z_{0}}{z_{1}-z_{0}}\right| \cdot \frac{n_{0}+n-\mathrm{I}}{n_{0}} \cdot \prod_{v=1}^{n-1}\left(\mathrm{I}+\frac{\mathrm{I}}{S\left(n_{0}+v-\mathrm{I}\right)^{2}}\right) \\
& \cdot \int_{\nu=1}^{n=1}\left(1-\frac{15}{t} \cdot \frac{\tau\left(z_{0},\left(n_{0}+v-1\right) \log k_{0}\right)}{\left(n_{0}+v-1\right) \log k_{0}}\right)
\end{aligned}
$$

hierin ist

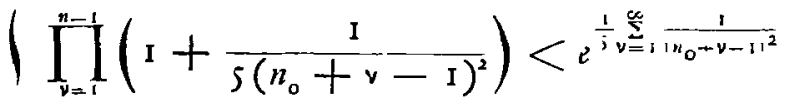

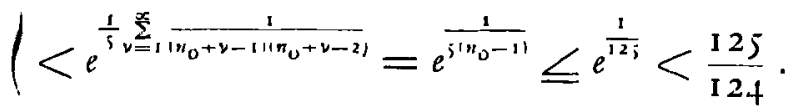

Also erhält man aus (22I) unter Benutzung von (222) und (220)

(223)

$$
\left\{\begin{array}{l}
<\left|\frac{f\left(z_{1}\right)-f\left(z_{0}\right)}{\left.z_{1}-z_{0}\right)-f\left(z_{0}\right) \mid}\right| \cdot\left|z_{n}-z_{0}\right|(n+n-\mathrm{I}) \cdot \frac{\mathrm{I} 25}{\mathrm{I} 24 \cdot 26} \cdot \frac{3 \varepsilon}{4 A_{1}^{2}} \cdot\left|\frac{z_{1}-z_{0}}{f\left(z_{1}\right)-f\left(z_{0}\right)}\right| \\
=\left|z_{n}-z_{0}\right| \cdot\left(n_{0}+n-\mathrm{I}\right) \cdot \frac{375}{\mathrm{I} 24 \cdot \mathrm{I} 04} \cdot \frac{\varepsilon}{A_{1}^{2}} .
\end{array}\right.
$$

Für alle Werte $z$, die der Bedingung

$$
\left|z-z_{0}\right| \leq\left|i_{n}-z_{0}\right|
$$

genügen, erhält man nach (20\%), (185) und (223)

$$
(225)\left\{\begin{array}{l}
\left|f(z)-f\left(\tilde{z}_{0}\right)-\frac{z-z_{0}}{z_{n}-z_{0}}\left(f\left(z_{n}\right)-f\left(z_{0}\right)\right)\right|<2 \mathrm{I} \cdot n\left(\left|z_{n}-z_{0}\right|\right) \cdot\left|f\left(z_{n}\right)-f\left(z_{0}\right)\right| \\
<\frac{21}{K_{0}\left(n_{0}+n-\mathrm{I}\right) \log k_{0}} \cdot\left|z_{n}-z_{0}\right| \cdot\left(n_{0}+n-\mathrm{I}\right) \cdot \frac{375}{124 \cdot 10_{4}} \cdot \frac{\varepsilon}{A_{1}^{2}} \\
<\frac{315}{12+\cdot 16} \cdot i_{i_{1}}-z_{0} \mid \cdot \frac{\varepsilon}{A_{1}^{2}} .
\end{array}\right.
$$


Man bestimme $r$ so, dass

(226)

$$
\frac{A_{1}}{2^{r-1}}>\left|z_{n}-z_{0}\right| \geqslant \frac{A_{1}}{2^{r}}
$$

Es wird infolgedessen

$$
\left|f(z)-j\left(z_{0}\right)-\frac{z-z_{0}}{z_{n}-z_{0}}\left(f\left(z_{1}\right)-f\left(z_{0}\right)\right)\right|<\frac{315}{12+8} \cdot \frac{\varepsilon}{A_{1}} \cdot \frac{1}{2^{r}} .
$$

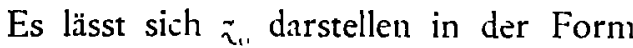

wo

(228)

$$
z_{0}=x_{1} z_{1}^{\prime r \prime}+x_{2} z_{2}^{\prime r \prime}+x_{i} i_{i}^{\prime \prime \prime},
$$

$$
\alpha_{1}+\alpha_{2}+\alpha_{3}=1
$$

und $x_{1}, x_{2}, \alpha_{\text {; }}$ reelle positive Zahlen. Ebenso lässt sich jeder Punkt des geraden Integrationsweges $z_{2}^{\prime r 1} \ldots i_{j}^{\prime r 1}$ darstellen in $\operatorname{der}$ Form

Wo

$$
z=\psi_{2} z_{2}^{\prime \prime \prime}+\psi_{3} z_{j}^{\prime r \prime} \text {, }
$$

(229)

$$
\psi_{2}+\psi_{3}=\mathrm{I}
$$

und $\dot{\sim}_{2}, \psi_{;}$; ebentalls reelle positive Zahlen sind. Es wird

$$
\begin{aligned}
z-z_{0} & =-\alpha_{1} z_{1}^{\prime \prime 1}+\left(\xi_{2}-\alpha_{2}\right) z_{2}^{\prime \prime \prime}+\left(\beta_{3}-\alpha_{3}\right) i_{3}^{\prime \prime \prime} \\
& =i_{1} z_{1}^{\prime r 1}+r_{2} z_{2}^{\prime r 1}+\gamma_{3} i_{3}^{\prime r 1},
\end{aligned}
$$

worin nach (228) und (229)

$$
\ddot{i}_{1}+\ddot{i}_{2}+\ddot{i}_{3}=0 \text {. }
$$

Also besitzen zwei der Grössen $\dddot{\jmath}$ das gleiche Vorzeichen, die dritte das entgegengesetzte. Es sei z. B.

dann ist

$$
\operatorname{sgn} \dddot{i}_{1}=-\operatorname{sgn} \dddot{i}_{2}=-\operatorname{sgn} \ddot{\imath}_{;} \text {, }
$$

(230)

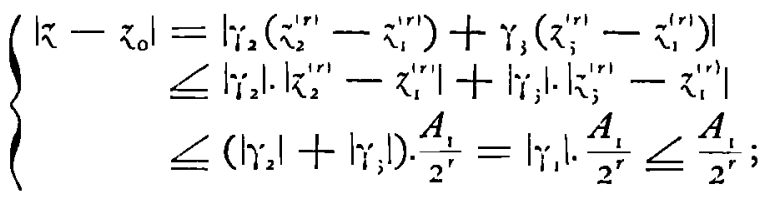

denn $\ddot{\imath}_{1}, \gamma_{2}, \gamma_{3}$ können als Differenzen zweier positiver Grössen absolut nicht grösser sein als die grössere der beiden. Also folgt fur alle Punkte des Integrationsweges von

$$
\int_{\left(a_{2}^{n}, a_{2}^{(n), u^{(n)}}\right.} f(i) d z
$$

aus (230) und (226), dass (22t) erfüllt, also auch (225) oder (227) anwendbar ist.

$\mathrm{Da}$ das Integral $\int f(z) d z$ über einen geschlossenen Integrationsweg verschwindet, wenn speziell $f(z)=\mathrm{I}$ oder $f(z)=z$, so ist nach (216) und (227)

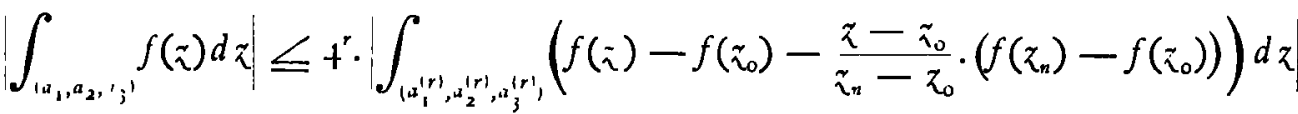

$$
\begin{aligned}
& \leq 4^{r} \cdot \int_{\left(x_{1}^{(r)}, u_{2}^{(r)}, a_{3}^{(r)},\right.}\left|f(z)-f\left(i_{0}\right)-\frac{z-z_{0}}{z_{n}-z_{0}}\left(f\left(z_{n}\right)-f\left(z_{0}\right)\right)\right| \cdot|d z| \\
& <4^{r} \cdot \frac{3 \mathrm{I} 5}{\mathrm{I} 24.8} \cdot \frac{\varepsilon}{A_{1}} \cdot \frac{\mathrm{I}}{2^{r}} \cdot 3 \frac{A_{1}}{2^{r}}=\frac{945}{992} \varepsilon<\varepsilon .
\end{aligned}
$$


Es war aber $\varepsilon$ eine heliebig kleine Zahl. Also ist

$$
\int_{\left(u_{1}, u_{2}, u_{3}\right)} f(z) d z=0,
$$

was zu beweisen war. $f(i)$ ist eine analytische Funktion, deren Ableitung in $T$ nicht rerschwinden kann, $d a$ an einer Nullstelle der Ableitung die Abbildung nicht winkeltreu wäre.

Zu bemerken ist noch, dass für jede analytische Funktion an einer Stelle, an der sie regulär ist und ihre Ableitung nicht verschwindet, $(185)$ tatsächlich erfüllt ist.

Nach $\left(18_{+}\right)$darf $\tau\left(\tilde{i}, \log \hat{\delta}^{-1}\right)$ speziell eine Konstante sein. Man kann also z. B.

setzen. Es sei nun

$$
\frac{\mathbf{I}}{K_{0}}-\tau=\frac{\mathrm{I}}{5}<\frac{\mathrm{I}}{K_{0}}
$$

$$
f(z)=\sum_{v=0}^{\infty} a_{\nu} z^{v}
$$

für $|z| \leq r$ absolut konvergent, wo $a_{1} \neq 0$ und $r$ kleiner sei als der wahre Konvergenzradius. Dann ist

Wenn nun

$$
\left.\frac{f\left(z_{2}\right)-f\left(i_{1}\right)}{z_{2}-i_{1}}=a_{1}+\sum_{\nu=2}^{\infty} \mid a_{\nu} \cdot \sum_{i=0}^{\nu=1} z_{1}^{\lambda} z_{2}^{\nu-1-\lambda}\right\} .
$$

so wird

$$
\left|\tilde{n}_{1}\right| \leq \delta \text { und }\left|\tilde{z}_{2}\right| \leq \delta
$$

$$
\left|\frac{f\left(z_{2}\right)-f\left(z_{1}\right)}{\tilde{i}_{2}-z_{1}}-a_{1}\right| \leq \delta . \sum_{v=2}^{\infty} v\left|a_{v}\right| r^{\nu-2}=\delta . G,
$$

wo $G$ eine von $\delta$ unabhängige Konstante ist. Also ist

mithin nach (23)

$$
\left|\frac{\mathrm{I}}{a_{1}} \cdot \frac{f\left(z_{2}\right)-f\left(z_{1}\right)}{z_{2}-z_{1}}-\mathrm{I}\right| \leq \delta \cdot \frac{G}{\left|a_{1}\right|},
$$

$$
\begin{gathered}
\left|\operatorname{sgn} \frac{f\left(z_{2}\right)-f\left(z_{1}\right)}{z_{2}-z_{1}}-\operatorname{sgn} a_{1}\right|=\left|\operatorname{sgn}\left(\frac{\mathrm{I}}{a_{1}} \cdot \frac{f\left(z_{2}\right)-f\left(z_{1}\right)}{z_{2}-z_{1}}\right)-\mathrm{I}\right| \\
<\sqrt{2-2 \sqrt{\mathrm{I}-\frac{\delta^{2} G^{2}}{\left|a_{1}\right|^{2}}}}<\delta H<\frac{\mathrm{I}}{5 \log \delta^{-1}}
\end{gathered}
$$

für alle hinreichend kleinen $\delta$. Hierin bedeutet $H$ eine passend gewählte, von $\frac{G}{\left|a_{\mathfrak{a}}\right|}$ abhängige, von $\delta$ unabhängige Konstante.

Berlin, den r9. Juli i 913.

ROBERT REMAK. 